\title{
A Versatile Method of Resolution Enhancement for Tactile Sensor Array Used as Synthetic Skin: Modeling and Implementation
}

Karen Flores De Jesus

West Virginia University

Follow this and additional works at: https://researchrepository.wvu.edu/etd

\section{Recommended Citation}

Flores De Jesus, Karen, "A Versatile Method of Resolution Enhancement for Tactile Sensor Array Used as Synthetic Skin: Modeling and Implementation" (2013). Graduate Theses, Dissertations, and Problem Reports. 454.

https://researchrepository.wvu.edu/etd/454

This Thesis is protected by copyright and/or related rights. It has been brought to you by the The Research Repository @ WVU with permission from the rights-holder(s). You are free to use this Thesis in any way that is permitted by the copyright and related rights legislation that applies to your use. For other uses you must obtain permission from the rights-holder(s) directly, unless additional rights are indicated by a Creative Commons license in the record and/ or on the work itself. This Thesis has been accepted for inclusion in WVU Graduate Theses, Dissertations, and Problem Reports collection by an authorized administrator of The Research Repository @ WVU. For more information, please contact researchrepository@mail.wvu.edu. 


\title{
A Versatile Method of Resolution Enhancement for Tactile Sensor Array Used as Synthetic Skin: Modeling and Implementation
}

\author{
Karen Flores De Jesus \\ Thesis submitted \\ to the Benjamin M. Statler College of \\ Engineering and Mineral Resources \\ at West Virginia University \\ in partial fulfillment of the requirements for the degree of \\ Master of Science \\ in \\ Mechanical Engineering \\ Marvin Cheng, Ph.D., Chair \\ Victor Mucino, Ph.D. \\ Konstantinos Sierros, Ph.D. \\ Department of Mechanical and Aerospace Engineering \\ Morgantown, West Virginia \\ Dec, 2013
}

Keywords: resolution enhancement, FSR, tactile sensor array, shape identification Copyright 2013 Karen Flores De Jesus 


\begin{abstract}
A Versatile Method of Resolution Enhancement for Tactile Sensor
Array Used as Synthetic Skin: Modeling and Implementation
\end{abstract}

Karen Flores De Jesus

Tactile sensors are one of the major devices that enable robotic systems to interact with the surrounding environment. In particular, modern assistive robotic systems need to carry out many human-like activities. Thus, it is desired to have sensor arrays that can acquire different environmental information just like human skin. In the past two decades, various types of tactile sensor arrays have been developed to acquire different physical properties, such as temperature, force, and geometric shapes. However, though the measurement of a single sensor can be accurate, the planar resolution is limited due to fabrication difficulties. This research aims to propose a mathematical model to describe the behavior of a tactile sensor based on experimental and statistical analyses. Moreover, to develop a versatile algorithm that can be applied to different tactile sensor arrays to enhance the limited resolution. With the proposed algorithm, the resolution can be increased up to twenty times if multiple measurements are available.

To verify if the algorithm can be used for tactile sensor arrays that are used in robotic system, a $16 \times 10$ force sensing array (FSR) is adopted. The force was first measured by a scanning data acquisition device integrated by a synthesized electronic circuit, DAQ station and an interface developed using MATLAB. The acquired two dimensional measurements were then processed by the Proposed Resolution Enhancement Algorithm (PREA) to enhance the resolution. The proposed algorithm can be used to improve the resolution for single image or multiple measurements. In this study, the developed MATLAB scripts can automatically identify the location of the targeted objects if multiple measurements are recorded. As a result, the resolution of the sensor is increased and it can be used as synthetic skin to identify accurate shapes of objects and applied forces. 
To my mother

Eva de Jesus

and

my sister and brothers

Gabriela, Daniel and Gerardo 


\section{ACKNOWLEDGMENTS}

I would like to express my deepest gratitude to my advisor, Dr. Marvin Cheng, for his mentoring, encouragement and persistent guidance. His assistance and ideas were essential for the completion of this research.

I would also like to thank my committee members, Dr. Victor Mucino and Dr. Konstantinos Sierros, for their time in reviewing this thesis and their advice to improve.

I am indebted to my colleagues and friends, Luis Alfonso Rivera, Andres Cavezza and Tanmay Mandal for their time and support along the master degree. 


\section{TABLE OF CONTENTS}

Page

DEDICATION .................. iii

ACKNOWLEDGMENTS. . . . . . . . . . . . . iv

LIST OF FIGURES . . . . . . . . . . . . . . . . . . xii

LIST OF TABLES . . . . . . . . . . . . . . . . . xiii

\section{Chapter}

1. INTRODUCTION .................... 1

1.1 Background........................................ 1

1.2 Problem Statement ................................. 2

1.3 Research Objective ..................................... 2

1.4 Document Outline.................................. 3

2. LITERATURE REVIEW. . . . . . . . . . . . . . . 4

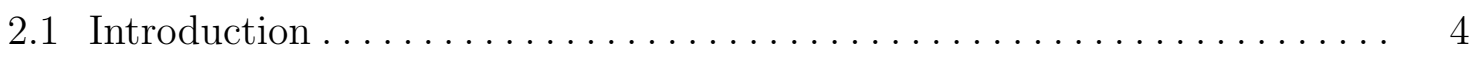

2.2 Developments of Artificial Skin......................... 5

2.3 Technologies of Tactile Sensors . . . . . . . . . . . . . . . . . . 7

2.3.1 Capacitive Sensors ............................ 7

2.3.2 Optoelectric Technology $\ldots \ldots \ldots \ldots \ldots \ldots \ldots \ldots \ldots \ldots \ldots$

2.3 .3 Piezoelectric Detector . . . . . . . . . . . . . . . . . . . . . . . 10

2.3.4 Resistive Sensors.................................. 10

2.3.5 Comparison among the Tactile Sensor Technologies ........... 11

2.4 Current Applications of Tactile Sensors . . . . . . . . . . . . . . . . 11

2.4.1 Industrial Applications ............................. 11

2.4.2 Medical Applications ................................. 12

2.4 .3 Mobile Devices ..................................... 13 
2.5 Tactile Sensors in Robotics .................................. 13

2.5.1 Applicable Sensors ............................... 15

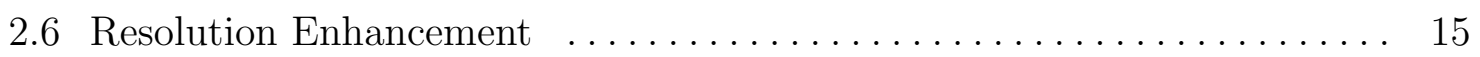

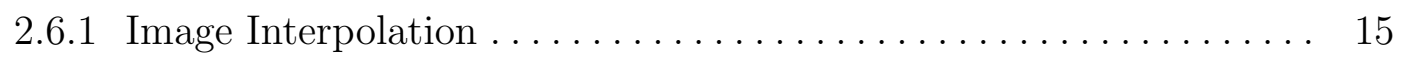

2.6.2 Super Resolution Algorithm ........................ 16

3. FSR: MEASUREMENTS AND MODELING . . . . . . . . . . 18

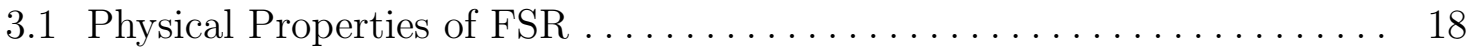

3.1 .1 Current Measurement ............................ 20

3.1 .2 Voltage Measurement............................... 21

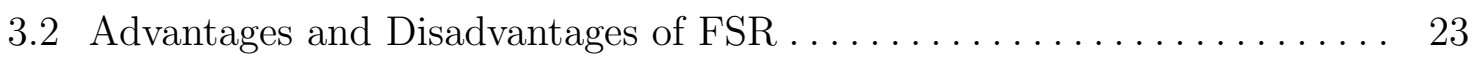

3.3 Experimental Platform .............................. 23

3.3 .1 Experimental Setup ........................... 23

3.3.1.1 Force Measurements of a Single FSR ............... 24

3.3.1.2 Force Measurements of a Thru mode FSR array ......... 25

3.3.2 Measurements and Data Collection..................... 26

3.3.2.1 Circuit Design of Sensor Array .................... 26

3.3.2.2 Data Acquisition Station ........................ 28

3.4 Statistical Analysis of the Measurements . . . . . . . . . . . . . . . . . . 29

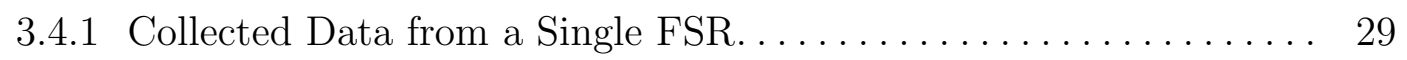

3.4.2 Collected Data from the FSR Array ................... 31

3.4.3 Location-Oriented Model ............................. 32

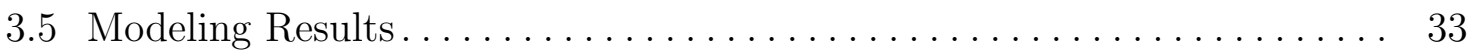

3.6 Model Validation....................................... 36

4. RESOLUTION ENHANCEMENT ALGORITHM . . . . . . . 39

4.1 Resolution Enhancement ............................. 39

4.2 Super Resolution Approach ............................... 41

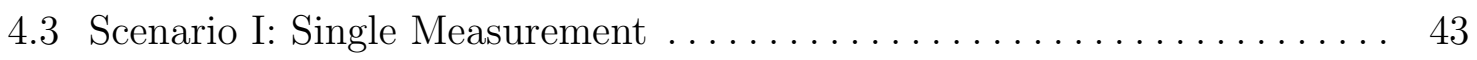

4.3 .1 Resolution Improvement ............................ 43

4.3 .2 Image Downsizing. .......................... 46

4.4 Scenario II: Multiple Measurements........................... 48

4.4.1 Method of Object Registration ..................... 48

4.4.2 Mathematical Approach .............................. 50

4.4.3 Example of Resolution Enhancement with Multiple Images ..... 52 
5. EXPERIMENTAL RESULTS. . . . . . . . . . . . 56

5.1 Resolution Enhancement of FSR Measurement ................. 56

5.2 Resolution Enhancement of a Single Set of Data................. 56

5.2 .1 Parameters of Image Quality $\ldots \ldots \ldots \ldots \ldots \ldots \ldots \ldots \ldots \ldots \ldots$

5.3 Resolution Enhancement of a Sensor Array ................... 61

5.3 .1 Processing of a Single Set of Data.................... 62

5.3.2 Processing of Objects with Multiple Sets of Data ............. 70

5.3.3 Reconstruction from Partial Information of Measurements....... 79

6. CONCLUSION. .................. 82

6.1 Summary of Research .............................. 82

6.2 Future Directions.................................. 84

BIBLIOGRAPHY .................. . 85 


\section{LIST OF FIGURES}

Figure 2.1 Tactile sensing activities in robotics $[1] \ldots \ldots \ldots \ldots \ldots \ldots \ldots \ldots$

Figure 2.2 Hands equipped with BioTact tactile sensors $[3] \ldots \ldots \ldots \ldots \ldots \ldots$

Figure 2.3 Nanowire array emulating syntethic skin under bending and rolling

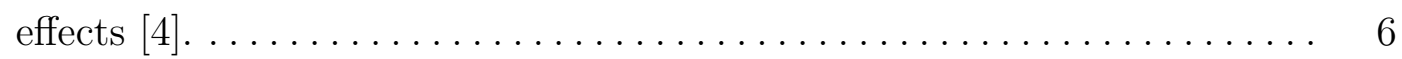

Figure 2.4 Proposed capacitive tactile sensor designed by Petropoulos et al.: a) Structure of the tactile sensor, b) Fabricated tactile sensor array. [12] . . 8

Figure 2.5 Working principle of an optical tactile sensor. There is an initial distance between the top surfaces and the LED-phototransistor, once a force is applied there is a deformation that changes the light reflection

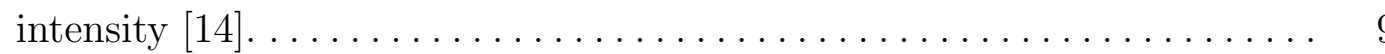

Figure 2.6 Resistive tactile sensor based on conductive polymers $[18] \ldots \ldots \ldots \ldots 11$

Figure 2.7 Photograph of a) Shadow Dexterous Hand [26], b) Internal Structure of

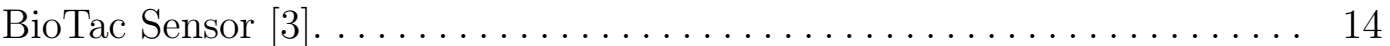

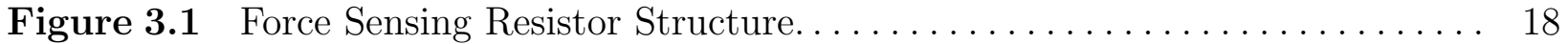

Figure 3.2 a) Layout of a Thru mode FSR array, b) Internal circuit configuration

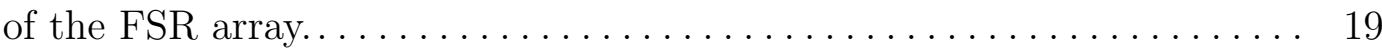

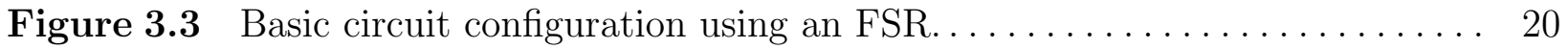

Figure 3.4 Circuit of a voltage divisor using an FSR $\ldots \ldots \ldots \ldots \ldots \ldots \ldots \ldots \ldots$

Figure 3.5 Experimental setup of the force measurement system . . . . . . . . . . 24

Figure 3.6 a) Experimental setup that is used to measure the resistance of a single FSR caused by the applied force. b) Correct location to place the gauge

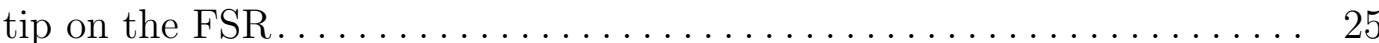

Figure 3.7 a)Experimental Setup that is used to measure the resistance of a Thru Mode array caused by applied force. b) Locations of the measured sensors. 
Figure 3.8 Schematic diagram of the implemented circuit to read the resistance

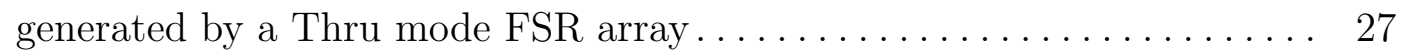

Figure 3.9 Graphic-user-interface of the developed MATLAB script. . . . . . . . 28

Figure 3.10 Experimental measurement using DAQ and the GUI: a) Display of measured outcome with GUI, b) physical positions of applied forces.... . 29

Figure 3.11 Collected data from a single FSR and the statistical model. . . . . . . 30

Figure 3.12 Collected data from a Thru mode FSR in terms of mean, and $+/-3 \sigma \ldots \quad 31$

Figure 3.13 Group map of a Thru Mode FSR array. . . . . . . . . . . . . . 33

Figure 3.14 Plots for every group of a Thru mode FSR array. . . . . . . . . . 34

Figure 3.15 Plot of the mean of the six groups, also the graph for each group is

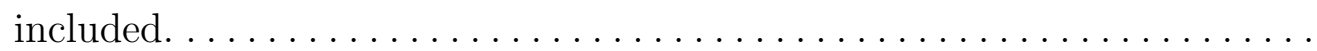

Figure 4.1 Schematic diagram of the data acquisition procedure . . . . . . . . 40

Figure 4.2 Schematic illustration of a low resolution block reconstructed to a higher resolution depending on the value $L$. The three blocks have the same high and width but the pixel density grows proportionally to the

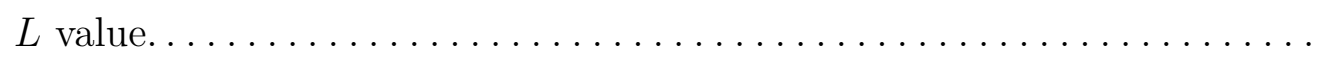

Figure 4.3 Application of the proposed resolution algorithm for surveillance purposes. a) The input data is a distorted face and b) high resolution image with a density of $681 \times 681$ pixels. The simulation time of the algorithm was 0.484 seconds...................... 44

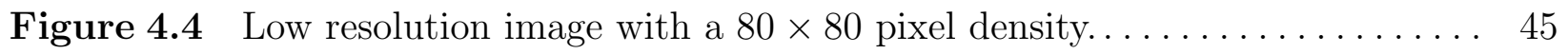

Figure 4.5 Enhancement of a low resolution image applying six different ratios $L=2, L=5, L=10, L=15, L=20$ and $L=30 \ldots \ldots \ldots \ldots \ldots$

Figure 4.6 Downsampling a high resolution image using two compression ratios: $L=25 \%$ and $L=10 \% \ldots \ldots \ldots \ldots \ldots \ldots \ldots \ldots \ldots \ldots \ldots \ldots \ldots \ldots \ldots \ldots \ldots \ldots \ldots$

Figure 4.7 Procedure to process a set of multiple shifted images. . . . . . . . . . 49

Figure 4.8 Set of nine images of a indented mark produced by a Berkovich tip... . . 52 
Figure 4.9 Set of nine images of a indented mark translated to the same coordinate. 53

Figure 4.10 Comparison between the a) original data $34 \times 34$ with b) the enhanced image $68 \times 68$ and c) the deblurred image $68 \times 68 \ldots \ldots \ldots \ldots \ldots \ldots 54$

Figure 4.11 Comparison of the 3D images between the a) original data $34 \times 34$ with b) the enhanced image $68 \times 68$ and c) the deblurred image $68 \times 68 \ldots \ldots 54$

Figure 5.1 Raw data of the original $512 \times 512$ sampled output one $(10 \mu \mathrm{m} \times 10 \mu \mathrm{m})$. The dotted square shows the selected area. . . . . . . . . . . . 57

Figure 5.2 Comparison between the data of sample 1: a) Original image; b) downsampled image $25 \%$; and c) the reconstructed image. . . . . . . . 57

Figure 5.3 Raw data of the original 512x512 sampled output two $(10 \mu \mathrm{m} \times 10 \mu \mathrm{m})$. The dotted square shows the selected area. . . . . . . . . . . . . 58

Figure 5.4 Comparison between the data of sample 2: a) Original image; b) downsampled image $25 \%$; and c) the reconstructed image. . . . . . . 58

Figure 5.5 Procedure to improve the resolution of the input data provided by a

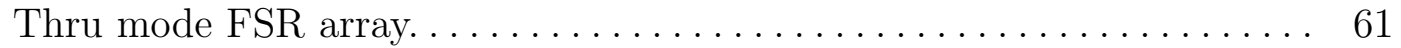

Figure 5.6 Annulus: a) Object on the top of the Thru mode FSR array and b) dimensions of the annulus............................

Figure 5.7 Results produced from a single image of an annulus: a) original sample; b) processed data with PREA where $L=10$; and c) the deblurred image. 63

Figure 5.8 Three dimensional images of the annulus of a) the processed data and b) the deblurred image. ...................... 64

Figure 5.9 Letter "L": a) Object on the top of the Thru mode FSR array and b) dimensions of the letter "L" ........................ 64

Figure 5.10 Results produced from a single image of the letter "L": a) original sample; b) processed data with PREA where $L=10$; and c) the

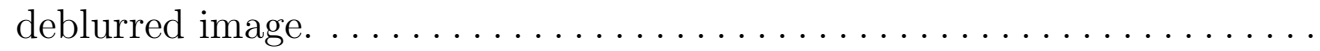

Figure 5.11 Three dimensional images of the letter "L" of a) the processed data and b) the deblurred image. . . . . . . . . . . . . . . . . . . 
Figure 5.12 Letter "V": a) Object on the top of the Thru mode FSR array and b)

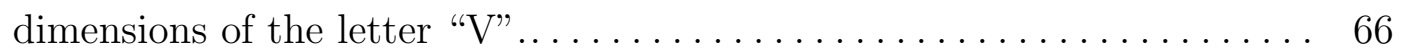

Figure 5.13 Results produced from a single image using the Letter "V": a) original sample; b) processed data with PREA where $L=10$; and c) the deblurred image. ........................ 66

Figure 5.14 Three dimensional images of the letter "V" of a) the processed data and $b)$ the deblurred image. . . . . . . . . . . 67

Figure 5.15 Letter "T": a) Object on the top of the Thru mode FSR array and b)

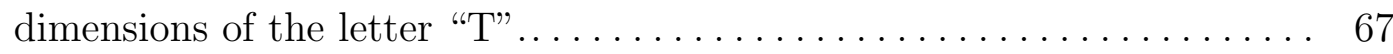

Figure 5.16 Results produced from a single image using the Letter "T": a) original sample; b) processed data with PREA where $L=10$ and c) the deblurred image. ............................ 68

Figure 5.17 Three dimensional images of the letter "T" of a) the processed data and $b)$ the deblurred image. ..................... 68

Figure 5.18 Letter "C": a) Object on the top of the Thru Mode FSR array and b)

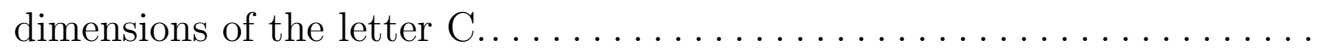

Figure 5.19 Results produced from a single image using the Letter "C": a) original sample; b) processed data with PREA where $L=10$; and c) the

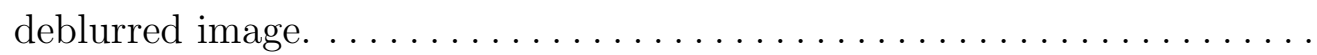

Figure 5.20 Three dimensional images of the letter "C" of a) the processed data

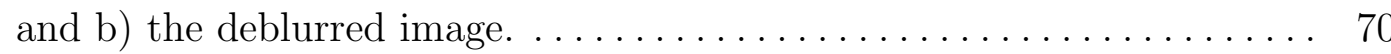

Figure 5.21 Images of nine measurements of an annulus using a Thru mode FSR

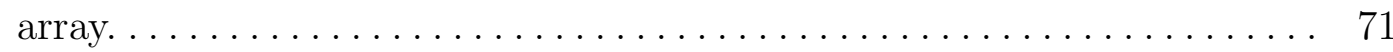

Figure 5.22 Results produced from multiple images of an annulus: a) averaged image; b) processed data with PREA where $L=10$; and c) the

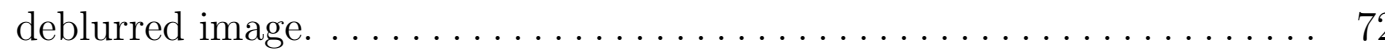

Figure 5.23 Three dimensional images of an annulus of a) processed data and b) the

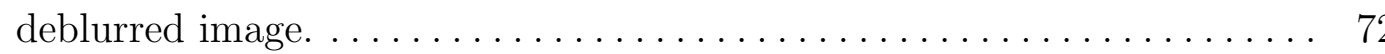

Figure 5.24 Set of 12 measurements of the letter "L" using a Thru mode FSR array. . 73 
Figure 5.25 Results produced from multiple images of the letter "L": a) averaged image; b) processed data with PREA where $L=10$; and c) the deblurred image. .......................... 74

Figure 5.26 Three dimensional images of the letter "L" of a) processed data and b)

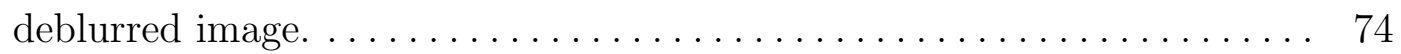

Figure 5.27 Set of 12 measurements of the letter "V" using a Thru mode FSR array. 75

Figure 5.28 Results produced from multiple images of the letter "V". a) averaged image; b) processed data with PREA where $L=10$; and c) the

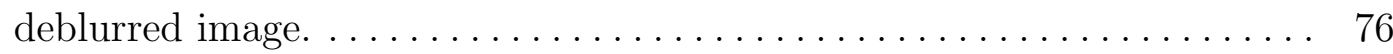

Figure 5.29 Three dimensional images of the letter "V" of a) processed data and b)

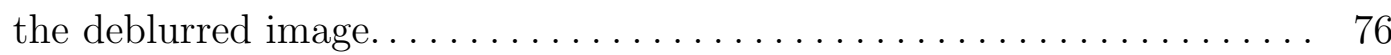

Figure 5.30 Set of 12 measurements of the letter "T" using a Thru mode FSR array.. 77

Figure 5.31 Results produced from multiples images of the letter "T": a) averaged image; b) processed data with PREA where $L=10$; and c) the

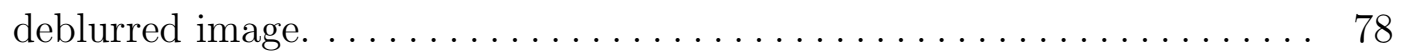

Figure 5.32 Three dimensional images of the letter " $\mathrm{T}$ " of a) processed data and b) the deblurred image. . . . . . . . . . . . . . 78

Figure 5.33 Set of 12 measurements of the letter "C" using a Thru mode FSR array.. 79

Figure 5.34 Results produced from multiple images of the letter "C": a) averaged image; b) processed data with PREA where $L=10$; and c) the

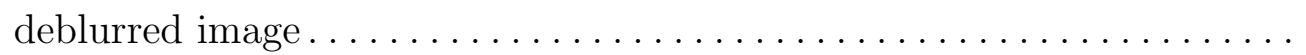

Figure 5.35 Three dimensional images of the letter "C" of a) processed data and b)

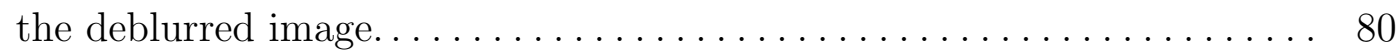




\section{LIST OF TABLES}

Table 2.1 Comparison between the tactile sensor technologies . . . . . . . . . . . 12

Table 3.1 Range of voltages using different additional resistors connected to a

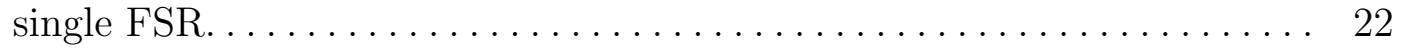

Table 3.2 Range of voltages using different additional resistors connected to a

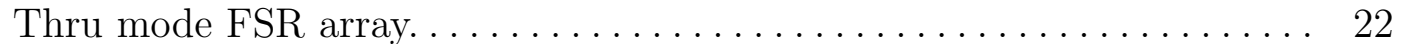

Table 3.3 Mathematical model of each group of a Thru mode FSR array. . . . . . 36

Table 3.4 Set of tables with the validation model corresponding to each group. The error percentage is based on the difference between the modeled resistance versus the physical measurement............... 38

Table 5.1 Performance comparison between the proposed enhancement resolution algorithm with the adaptive sampling algorithm. . . . . . . . . 60 


\section{CHAPTER 1}

\section{INTRODUCTION}

\section{$1.1 \quad$ Background}

A goal in robotic field nowadays is to develop humanoids, robots able to perform activities similar as human beings. The interaction between the robotic systems and external environment has been facilitated by the implementation of various sensors, which can provide important data such as force, position and shape. Recent studies have demonstrated how to provide robot systems human-like sensing with artificial skin. With the synthetic skin, a robotic system can sense force, texture, pressure and in some cases temperature.

This work aims to present a complete tactile system that emulates a synthetic skin which can detect applied force and the geometric shape of the targeted object. This system is based on Force Sensing Resistor (FSR). An FSR is a resistor that varies its resistance in relation to the applied force. This work proposes to use an array of FSR as the tactile device because of its simple work principle and low cost. The adopted array includes 160 sensor as a 16x10 matrix. The mathematical relation between the force and resistance is modeled by the experimental results and a corresponding statistical analysis. The measurements of the tactile sensor are first acquired by an electronic circuit and then connected to the data acquisition station. The major drawback is that the resolution of a FSR array and its repeatibility are really poor. The measurement of a particular force cannot guarantee a consistent resistance readout. With the unstable measurement, identification of geometric shapes cannot be accurate. As a result, in order to improve the resolution of the tactile sensor array, an image processing method is proposed. This Proposed Resolution Enhancement Algorithm (PREA) is capable to improve the image quality of a low resolution image accurately. 
With PREA, the geometric shape of the targeted object from the measurement of the adopted FSR array can be easily identified.

In short, the goal of this project is to propose a robust an effective tactile system that emulates synthetic skin. This proposed artificial skin system has to be able to provide data of force distribution and shape of the targeted object.

\subsection{Problem Statement}

In industrial applications, robots are able to grasp just the kind of object that they were programmed for. The objects need to have identical shape, material, position and even orientation while the robot is in operation. As the physical condition of the targeted object changes, the robotic arm fails to grasp the object. In many cases, robots programmed to follow a certain trajectory cannot respond to sudden changes unless a feedback controller with measurements from the surrounding environment can be available. The interaction with the external environment can be implemented by adopting adequate sensors. These sensors required for interaction environments include force distribution, position, weight and shape identification. Therefore, a system similar to human skin is necessary.

Many studies have been investigated to develop a synthetic skin which can provide information mentioned above. However, though the measurement can be accurate, these innovative sensors are usually complex and expensive. Thus, a simpler and low cost approach is desired.

\subsection{Research Objective}

The general objective of this research is to propose the implementation of a low cost tactile system that can measure force, position, weight and shape of a targeted object. Among all different options, FSR has the advantage of low cost and easy implementation of supporting circuits. Thus, a Thru mode FSR array is adopted in this research. 
With the FSR array, four major objectives need to be fulfilled. They are,

1. Identify a mathematical model of the tactile sensor based on experimental results and statistical analysis;

2. Develop an effective scanning circuit which can accurately read all the sensor measurements within a reasonable time;

3. Implement a DAQ station with software interface that can be access easily by users ; and

4. Develop a resolution enhancement method to improve the image quality acquired by the FSR.

\subsection{Document Outline}

The research is divided into six chapters. Chapter 1 is the introduction of this research. Chapter 2 reviews the literature of current technologies about tactile sensor and super resolution approaches. Chapter 3 explains the experimental procedure and statistical analysis to identify the mathematical model for a Thru mode FSR array. This chapter also discusses the design of the supporting circuit, DAQ station and software development. Chapter 4 introduces a Proposed Resolution Enhancement Algorithm (PREA) which changes the resolution of acquired measurement with an image processing method. In this chapter, the performance of the proposed method was verified with different scenarios. Chapter 5 shows three different sets of results of acquired measurements and processed images. Finally, Chapter 6 concludes this research and discusses the future directions. 


\section{CHAPTER 2}

\section{LITERATURE REVIEW}

\subsection{Introduction}

It is an essential ability that human beings can touch and sense the surrounding environment. To imitate these capabilities in a robotic system, tactile sensing is necessary to perform three important activities: manipulation, exploration and response [1]. The activity of manipulation is analogous to the capability of grasping, holding and moving any kind of object, regardless to its shape or material. The activity of exploration allows human being to acquire some important tactile information, such as temperature, hardness and texture. The activity of response is the most important one since this activity reacts to certain external events or forces. For instance, humans can hold a piece of egg or a heavy stone by just processing information by means of touching and sensing. Figure 2.1 illustrates the three tactile sensing activities in robotics.

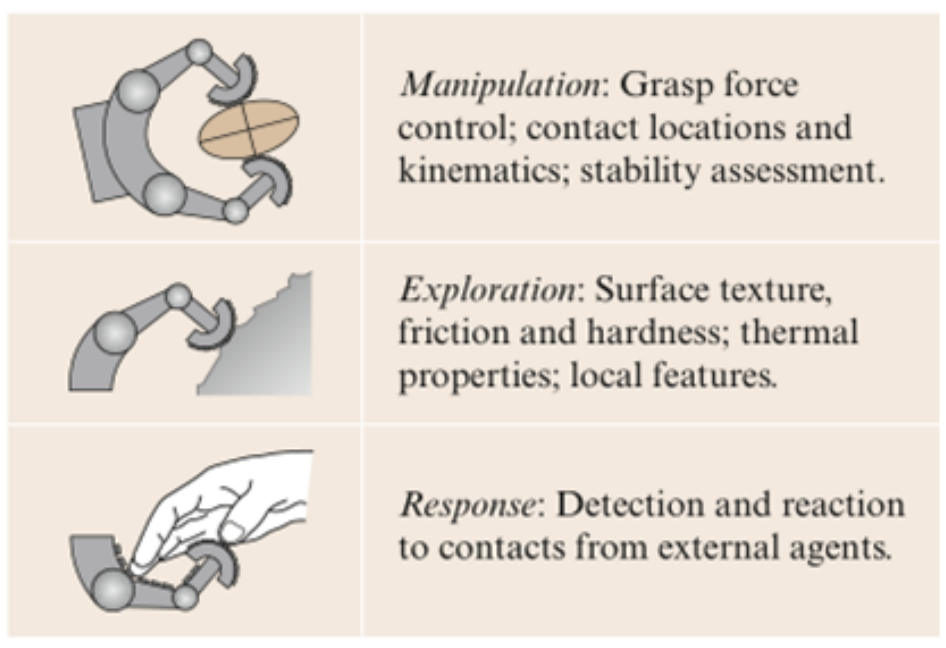

Figure 2.1 Tactile sensing activities in robotics [1]. 
In the past decade, the applications of robotics systems have extended from the area of industrial applications to medical surgery, agricultural tasks and service robots at home. For the robot that need to interact with human beings, these devices require to perform movements similar to human beings. To mimic human movements, these devices need to have a feedback similar to human skin to emulate the sense of touching. In order to increase the autonomy of a robotic entity, some researchers have conveyed on the idea of equipping robots with tactile sensors. Based on Lee and Nicholls [2] a tactile sensor is a "device that can measure a given property of an object or contact even through physical contact between the sensor and the object". Therefore, an approach to emulate synthetic human skin is by the development and implementation of thin layers of tactile sensor arrays.

\subsection{Developments of Artificial Skin}

To provide tactile capability for different applications of robotic systems, various types of sensor have been developed. Recent research have demonstrated great advantage to include tactile sensors in robot systems. For instance, in 2008, SynTouch LLC has started to manufacture the sensor named BioTac, which allows robots to imitate the human sense of touch. This device also emulates the touch of human fingertip. Figure reffig: biotac shows a robotic hand which integrates BioTac tactile sensors to hold a fragile surface [3]. In [4] a design of an artificial skin based on nanowires is presented, which is a sensor array of active components that can be used to identify a wide range of pressures. This tactile sensor (see Figure 2.3) has demonstrated great reliability and robustness after almost 2,000 bending cycles.

Currently, different approaches have been investigated to develop a new tactile sensor to imitate the human skin. For example, Mannsfeld et al. [5] proposed a new type of sensor array constructed with micro-structured films integrated as organic field transistor with high sensitivity and minimum time response. One of the most recent technology of tactile sensor was made by Segev-Bar et al. in 2013 [6]. They designed a flexible sensor based 

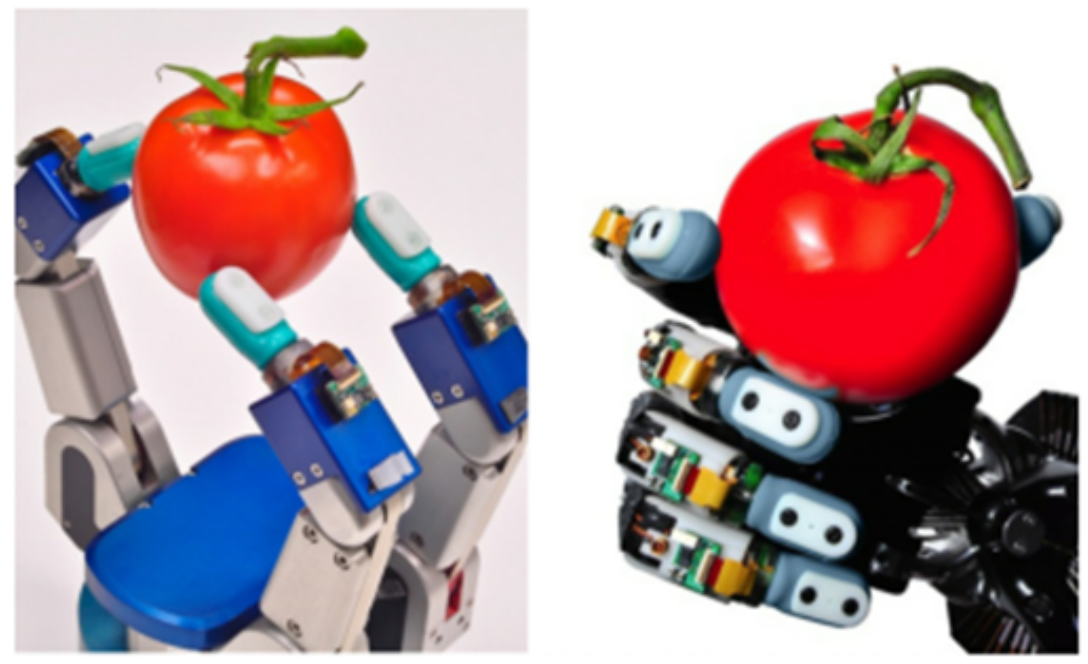

Figure 2.2 Hands equipped with BioTact tactile sensors [3].

on monolayer-capped nanoparticles that greatly improves the repeatibility of this type of sensor.
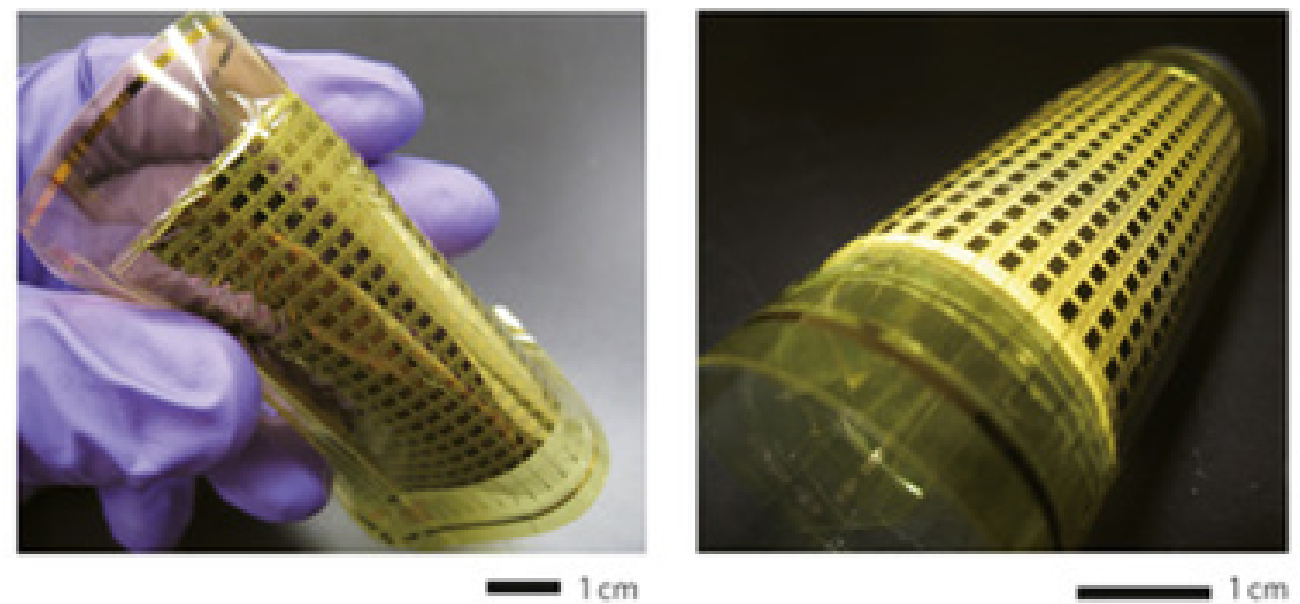

Figure 2.3 Nanowire array emulating syntethic skin under bending and rolling effects [4].

However, even though important advances respect to tactile sensing technologies have been made so far, it is still a long way to go to achieve the goal of ideal artificial skin that 
is similar to real human skin. In particular, though many physical properties, such as force, pressure, temperature and texture of material can be detected by various of tactile sensor, a sensor that can precisely identify geometric shapes of targeted objects still need to be investigated.

\subsection{Technologies of Tactile Sensors}

Tactile sensors can be classified into different categories based on the adopted technologies or working principles. Among these technologies tactile sensors can be classified as resistive, capacitive, optoelectric and piezoelectric [2] [7] [8] [9] [10]. These common types of sensors are explained in the following sections. A comparison among them is listed in table 2.1 .

\subsubsection{Capacitive Sensors}

Tactile sensors made of capacitive technology can measure applied force by sensing the change of capacitance. These type of sensors are mainly composed of two parallel plates of a certain area $\mathrm{A}$ and a dialectic material sandwiched between the two plates. The capacitance is measured by the relation $C=\varepsilon_{0} \varepsilon_{r} A / d$. Where $C$ is the capacitance, $\varepsilon_{0}$ is the dielectric constant $\left(\varepsilon_{0} \simeq 8.854 * 10^{-12} \mathrm{Fm}^{-1}\right), \varepsilon_{r}$ is the dielectric constant of the material between the plates, $A$ is the overlapped area of the two plates and $d$ is the distance between the plates. Once a force is applied to the sensor, the distance $d$ between the plates gets reduced. As a result, the capacitance increases. Thus, the relationship between applied force and capacitance can be obtained.

In [11] a tactile sensor based on the variation of capacitance between two electrodes was designed and fabricated, which can be used to measure external forces and also torques. The important highlight about this sensor is that it has a small sensing area of $5 \mathrm{~mm} \times 5 \mathrm{~mm}$ but a wide range of forces can be measured, which is $50 \mathrm{~N}$. 
In [12], Petropoulos et al. demonstrate the development of a capacitive sensor made of flexible organic material able to measure applied pressures. This sensor is composed by two electrodes and a spacer. Once they are joint together, there is a sealed cavity between the electrodes. When a pressure is applied to the sensor, the cavity yields corresponding deformation which changes the capacitance as illustrated in Figure 2.4.

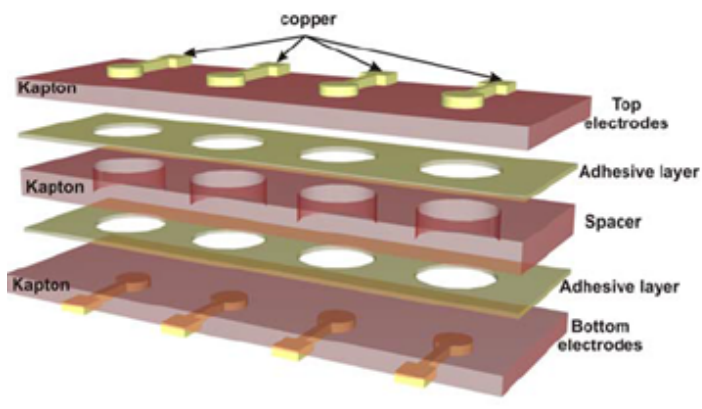

(a)

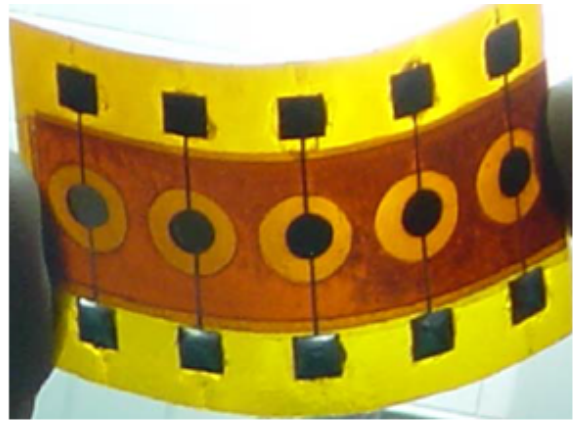

(b)

Figure 2.4 Proposed capacitive tactile sensor designed by Petropoulos et al.: a) Structure of the tactile sensor, b) Fabricated tactile sensor array. [12]

In [13], the design of a capacitive flexible polyimide force sensor was proposed. The distance between the bottom and top electrode is as thin as 5 micrometers. Due to the change of distance between the electrodes and the thickness of the insulation layer, the corresponding capacitance is changed and hence translated to applied force. The developed sensor demonstrates high durability because it can be used to detect force close to $3 \mathrm{kN}$ without any damage. The sensor also shows an excellent linear behavior without hysteresis phenomenon when it is working within a range of $1 \mathrm{kN}$. 


\subsubsection{Optoelectric Technology}

Other than capacitive type of tactile sensors, many different types of sensors have also been studied, such as optoelectric sensor.

Tactile sensors based on optoelectric technologies are mainly integrated by a light source and a photodetector. The basic idea of such kind of sensor is that light reflected on a surface is captured by the photodetector. When the surface is moved due to an applied force, the intensity of reflected light changes as well, which vary the generated current or resistance depending the adopted photodetector.

In [14], a complete design of a tactile sensor based optical technology is presented shown as Figure 2.5. The sensor consists of a deformable layer which has reflective surfaces and an array of optoelectronic devices, LED-phototransistor. Once a force is applied on the surface of the layer, the distance between the reflective surfaces and the optical detector changes. In order to find the relationship between the force and the deformation of reflection, an extensive and complex finite element analysis need to be implemented. Typically, this kind of data is processed by neural networks.

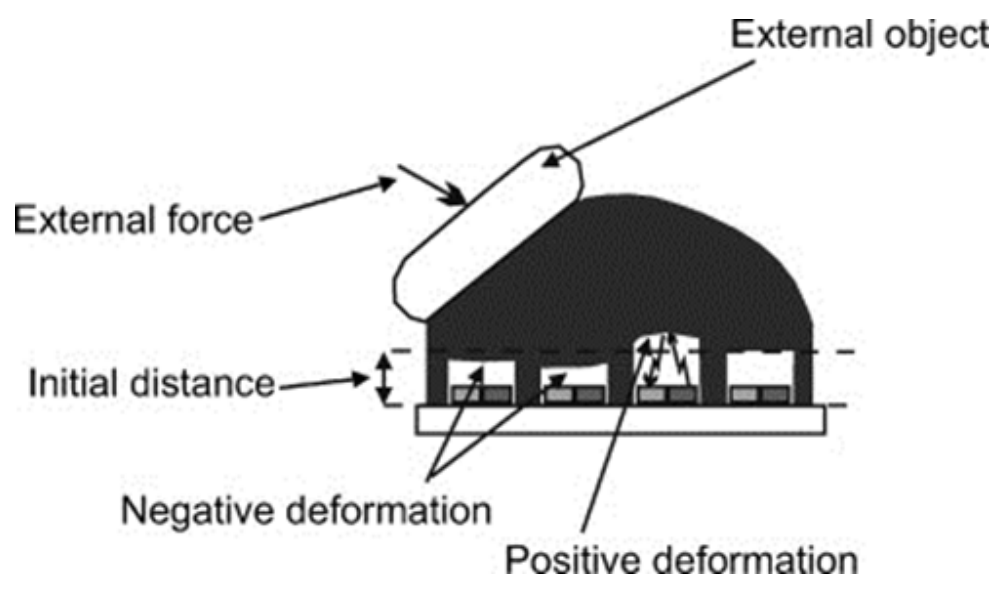

Figure 2.5 Working principle of an optical tactile sensor. There is an initial distance between the top surfaces and the LED-phototransistor, once a force is applied there is a deformation that changes the light reflection intensity [14]. 


\subsubsection{Piezoelectric Detector}

Piezoelectric components are also widely used as a force sensor. When a piezoelectric component is deformed, the output voltage level is changed. This property makes it a great material to detect force applied to a piezoelectric component. Certain materials, such as crystal, ceramics and polymers, are typically used for fabricating a piezoelectric component. Nowadays, one of the most popular material used for the design of piezoelectric tactile sensors is Polyvinylidene Fluoride (PVDF). PDVF is widely used due to its excellent flexibility, stability and thickness. Based on these superior properties, tactile sensors have been designed [15] [16] [17].

\subsubsection{Resistive Sensors}

A tactile sensor based on resistive technology can measure an applied force by detecting its change of resistance. Depending on adopted technology used to manufacture resistive tactile sensor, it can be classified into several categories. They are: 1) piezoresistive sensor; 2)strain gauge, 3)conductive polymer; and 4)conductive elastomer.

Currently, the most used technologies applied to resistive tactile sensors are conductive polymers and conductive elastomers. For instance, in [18] the development of an artificial skin has been investigated by Cheng et al. The proposed tactile sensor was an $8 \times 8$ array, as shown in Figure 2.6(a). This sensor was made of spiral electrodes and tactile sensing elements of conductive polymer which can vary their resistance with respect to the applied force. Two spiral electrodes are aligned perpendicularly without making any contact and a layer of conductive polymer is placed between the two electrodes. The advantage of this design is that it is highly stretchable which makes it a convenient sensor applied to various types of surfaces. Figure 2.6(b) illustrates the bending mode of the sensor and its stretchability. 


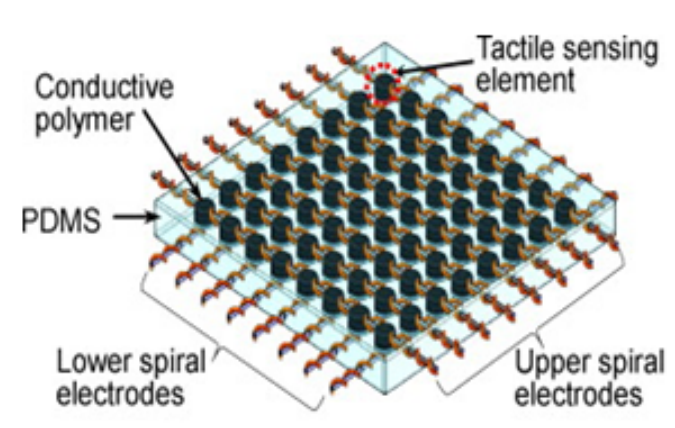

(a)

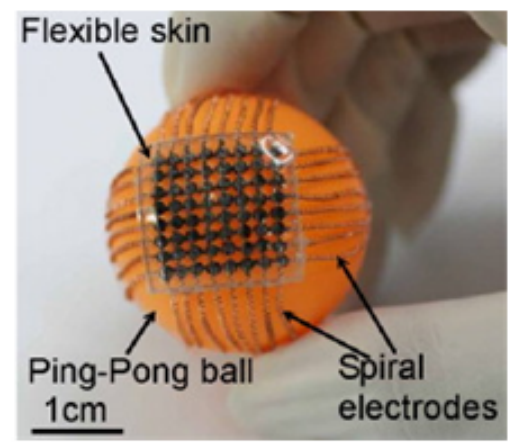

(b)

Figure 2.6 Resistive tactile sensor based on conductive polymers [18].

Another commonly investigated resistive tactile sensor is Force Sensing Resistor (FSR), which is made of two layers of polymer. FSR has the advantages of low cost and excellent flexibility compared with other sophisticated sensors. The supporting circuit is easy to design and implement as well. However, the size of such kind of sensor limits the applicable number of sensors within a given area. Further discussion of FSR is found in Chapter 3.

\subsubsection{Comparison among the Tactile Sensor Technologies}

Different technologies used by different types of tactile sensors have certain advantages and disadvantages. Table 2.1 summaries the features of commonly used technology and their upsides and downsides. Detailed reviews of tactile sensors can be found in [9] [10].

\subsection{Current Applications of Tactile Sensors}

\subsubsection{Industrial Applications}

Tactile sensor technology has been widely used by the industrial sector specially in automotive area where force feedback is extremely critical. For instance, Pressure Profile Systems, Inc. has developed a system that can measure the dynamic contact pressures 


\begin{tabular}{|c|c|c|}
\hline \hline Technology & Advantages & Disadvantages \\
\hline \hline \multirow{2}{*}{ Capacitance } & Good frequency response & Amount of noise \\
& High resolution & Complex external circuits \\
& Large dynamic range & Hysteresis \\
& Easy to manufacture & Undesirable frequency response \\
Resistive & Low to manipulate & Low Sensitivity \\
& Minimum noise effect & Low repeatability \\
\hline \multirow{3}{*}{ Optoelectronic } & High spatial resolution & Size and robustness \\
& Design simplicity & Slow response time \\
& Reliability & High impedance \\
Piezoelectric & Good frequency response & Unable to measure static forces \\
& & Limited to measure dynamic forces \\
\hline
\end{tabular}

Table 2.1 Comparison between the tactile sensor technologies

of tires. This system was designed as a $48 \times 40$ tactile sensor array with a resolution of $4 \mathrm{~mm}$. The fastest sample rate is approximately $420 \mathrm{kHz}$. The system has been used in the tire industry for applications such as suspension testing, tire design and performance, tire footprints pressure and tread evaluation. This system can also work under the conditions of high temperature and speed [19].

\subsubsection{Medical Applications}

Nowadays, due to the fast evolution of corresponding technologies, robotic science is not only growing in industrial sectors but also in new evolving areas, such as medical applications. Tactile sensors can be found in different medical studies that are currently implemented and functioning. For instance, tactile sensor technology has been applied for breast cancer detection. SureTouch, is a product that allows medical staff to detect any abnormal mass under the skin breast. This device can detect abnormal bodies up to $5 \mathrm{~mm}$. The most important fact is that it does not cause any pain to the patient. This specific device is an array with $12 \times 16$ tactile sensors. The acquired signal is processed by a software 
that shows different breast sections and displays the anomalies using a colormap scale [20].

Another use for tactile sensors in medical applications is body mapping. Body mapping can be applicable to body pressure system in automotive seats and mattresses. This kind of sensor can read different pressures that the body exhibits under a certain posture, podiatry and seating design. These kind of devices provide information of the posture which is a valuable data to detect injuries, to assist a patient and/or to improve the ergonomic function or rehabilitation [21].

\subsubsection{Mobile Devices}

One of the most common application of tactile sensor is the touch sensitive screen that can be found in mobile devices like smartphones, tablets, computers, laptops, game consoles and flat screen. The common technology of a touch screen can be resistive [22], surface acoustic wave(SAW) [23] or capacitive [24]. Nowadays, the most used technology is the capacitive due to allows multi-touch without creating any erratic problem. Newest technology integrated by iPhone is the capacitive technology. However, the capacitors are arranged as a grid which allows to have an array of tactile points. Each node generates its own signal once it is touched. This feature allows multi-touching, dragging, zooming-in, zooming-out and other features [25].

\subsection{Tactile Sensors in Robotics}

The desire to design and manufacture a new generation of robots that can interact with human beings has resulted in many research works based on the development and

implementation of advanced tactile sensors capable to read physical properties. Some of these proposed tactile technologies have been implemented in advanced robotic hands and humanoids. For instance, Shadow Robot Company manufactures the Shadow Dexterous Hand, which is currently the robotic arm closest to a real human hand [26]. It counts with 
20 degrees of freedom and each of the fingertips is equipped with the advanced tactile sensor fabricated by SynTouch LLC, BioTac [3]. These features allow Shadow Hand not only to emulate the movements of human hands but also to sense specific properties such as: texture, temperature and force vibrations.

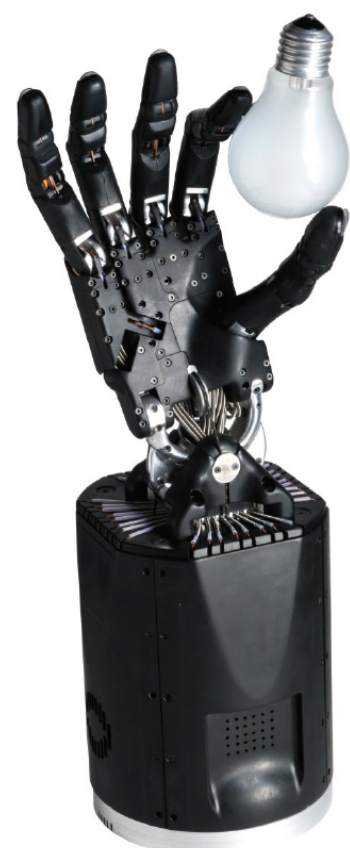

(a)

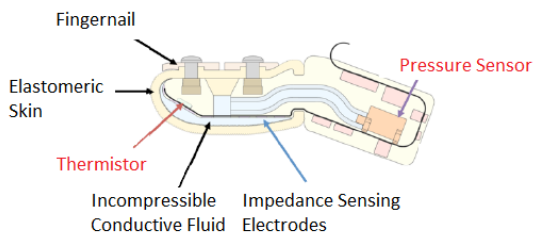

(b)

Figure 2.7 Photograph of a) Shadow Dexterous Hand [26], b) Internal Structure of BioTac Sensor [3].

Hiroyasu Iwata and Shigeki Sugano in [27] present the design of a human symbiotic robot named TWENDY-ONE. This advance humanoid is capable to grasp and hold objects of unknown weight, shape and stiffness. The surfaces of the palms and fingertips are integrated with six-axis force tactile sensors. The amount of tactile points is approximately 241 per each hand. This robot has been proposed to be applied as an assistive robot to help elderly people to move from one place to another. 


\subsubsection{Applicable Sensors}

In the applications mentioned above, tactile sensors are implemented as a certain number of single sensors located at certain locations or as an array of multiple elements. Most of the sensor are based on the capacitive and resistive technology. Even though the most advanced tactile sensors are capable to get accurate data from the environmental surrounding, they are still not able to produce a reliable image for object identification purposes. This is due to the low spatial resolution of sensors.

Nowadays, in order to improve the performance of tactile sensors, many innovative researches have been investigated. However, tactile sensors still show a low spatial resolution. Thus, a technique capable to increase the resolution of tactile devices and provide finer images is highly needed. One of these techniques is the application of image resolution methods to enhance low resolution images provided by imaging devices. Different methods such as image interpolation and super resolution algorithm have been investigated to improve low resolution images to high resolution.

\subsection{Resolution Enhancement}

\subsubsection{Image Interpolation}

Image interpolation is the process of calculating the values of intermediate pixels between the known pixels of certain low resolution (LR) image [28]. This method is commonly divided in different techniques such as bilinear interpolation, nearest neighbor interpolation, bicubic interpolation and adaptive cubic spline. These techniques are highly used in image processing to enhance the image resolution.

Many research studies using these techniques for the development of new algorithms have been reported. For instance, the existing Bicubic Convolution (BC) algorithms conveys low resolution images into sharper edges images. However, they produce some undesirable 
results when some textures details need to be preserved. In order to solve this, Zhou Dengwen in [29] proposes a new algorithm based on Bicubic Convolution (BC) Interpolation, which can produce sharp edges as well as texture details. The algorithm works as the basic ones in the case a missing pixel is on a strong edge. However, the algorithm can greatly improve image quality if the pixel is located on a weak edge. In this case, the pixel is interpolated using two orthogonal directional BC interpolation.

\subsubsection{Super Resolution Algorithm}

Super resolution or (SR) algorithm is a widely investigated method in image processing field. This is because it can improve the limited resolution images acquired by low cost imaging devices, such as cameras, cell phones and recently tactile sensors. A super resolution method can be implemented by processing multiple low resolution, blurred and downsampled images from the same targeted area or environment in order to produce a high resolution output. Recently, SR algorithms are applied to specific fields, such as: satellite data, surveillance images and medical imaging.

Chu et al. in [30], proposed a super resolution algorithm using a gradient-based adaptive interpolation. This approach not only takes into account the distance between the pixels but considers the local gradient of the low resolution image. The importance of the pixels is determined by the value of the gradient. This also ensures to preserve the important information and to obtain a high resolution image with defined edges. This method is suitable to work in real time applications due to its low computational work.

Even though most of the SR methods need to process multiple images in order to generate the desirable high resolution image. There are some approaches that only need a single low resolution image. For instance, Yang et al. in [31]. present a super resolution method applied to a single image using sparse representation. The algorithm consists of 
finding the sparse representation of a single targeted low resolution image. Based on the coefficients acquired from this representation, the algorithm can produce a high resolution image. The advantages of this approach are that it can enhance the resolution by using only a single image and it is robust to noise.

The super resolution algorithm has demonstrated a better performance compared with the conventional techniques for image processing. For this reason, this method is researched and it is also one of the most applied methods used to enhance low resolution in imaging devices.

This research proposes a new algorithm that can be classified as a super resolution technique due to it uses multiple low resolution images to generate a high and enhance output. This proposed algorithm was tested using downsampled and nanoscale images to verify its performance. Then, it is applied to enhance the output images generated by a tactile sensor array. This algorithm allows to enhance the images and therefore to identify the shape of a targeted object. 


\section{CHAPTER 3}

\section{FSR: MEASUREMENTS AND MODELING}

\subsection{Physical Properties of FSR}

A Force Sensing Resistor (FSR) is a resistive sensor that has been used as tactile sensor in engineering applications. This type of sensor changes its internal resistance corresponding to the applied force. The relationship between applied force and output resistance is inversely proportional, which signifies that the resistance gradient decreases as applied force increases. Typically, an FSR device is mainly composed by two substrates of resistive polyester, which represent the top and the bottom circuit, and a spacer sandwiched between these two surfaces. Physically, material and geometrical specifications of the spacer and the layers determine the force necessary to make contact between the two substrates and hence to start varying the resistance. Figure 3.1 illustrates the layout of a single FSR manufactured by Sensitronics, LLC [32].

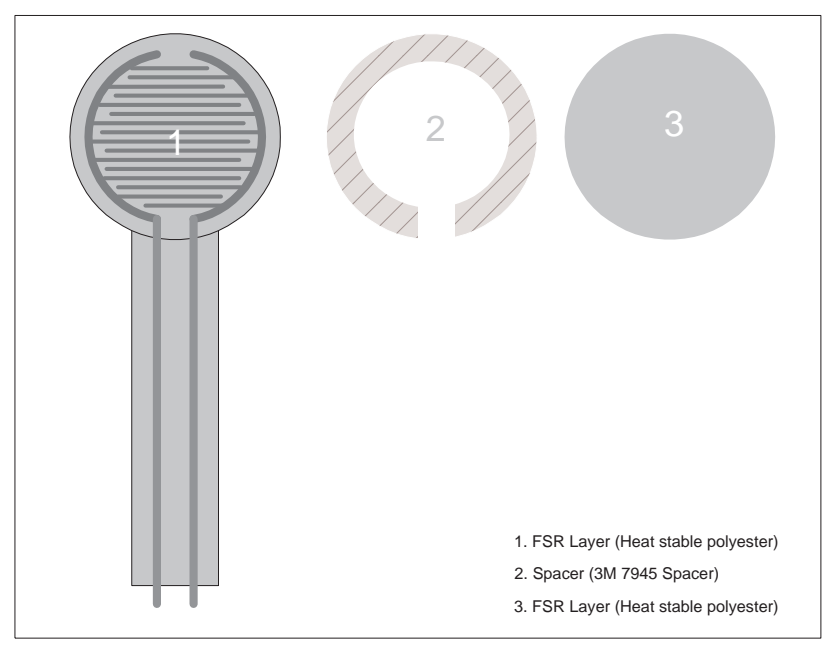

Figure 3.1 Force Sensing Resistor Structure. 
FSR can be manufactured as a single sensor to measure applied force and also an integration of multiple FSR can be synthesized as an array of a fixed number of sensors to measure force distribution. For instance, Sensitronics, a provider of tactile devices, supply two types of FSR arrays: Thru mode and Shunt mode. Shunt mode integrates 60 sensors accommodated in 10 rows by 6 columns. Thru mode array includes 160 sensors arranged as 16 rows by 10 columns. Both sensor array were studied in this research. From the experimental results the difference of output resistance between these two modes is minimum. Therefore, a Thru mode FSR array is adopted in this document. The configuration of a Thru mode FSR Array is shown in the Figure 3.2.

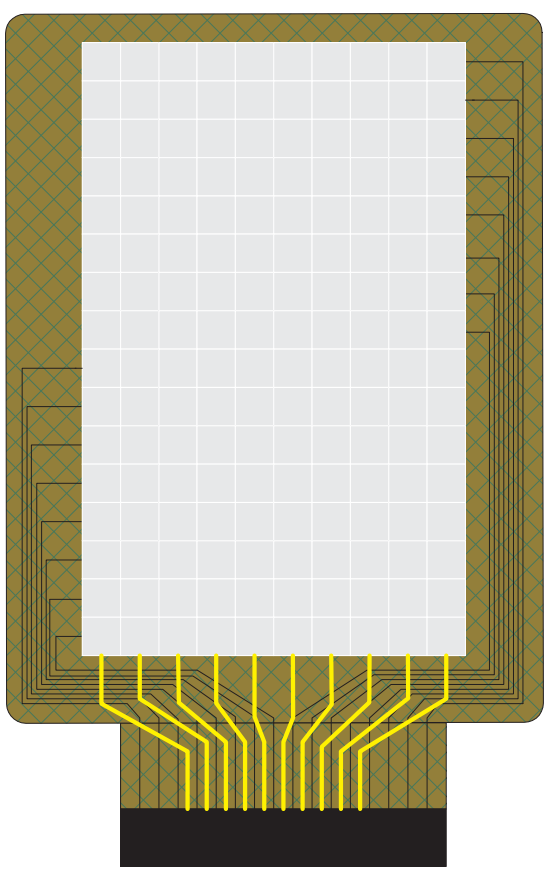

(a)

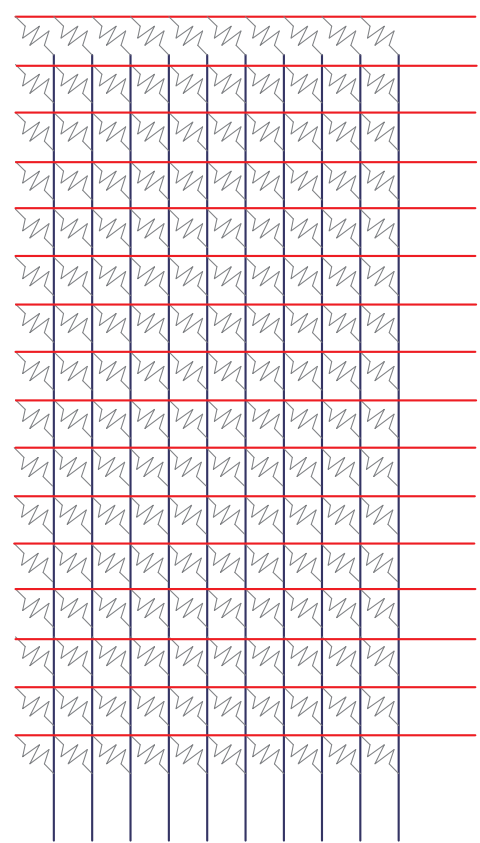

(b)

Figure 3.2 a) Layout of a Thru mode FSR array, b) Internal circuit configuration of the FSR array. 


\subsubsection{Current Measurement}

As a force applied on a FSR sensor, the resistance is changed. The change of resistance of an FSR can be measured by using a regular multimeter. However, it is difficult to measure 160 resistive outputs if a Thru mode FSR array is used. A supporting circuit that processes automatically all the readings is necessary. Based on the data acquisition device adopted, the resistive changes cannot be measured directly. Instead, a FSR can be connected to a circuit that acquires either current or voltage. For instance, if we consider a simple circuit shown in Figure 3.3 where an FSR can be used to acquire the change of current. Based on Ohm's Law the current can be obtained by having a power source with fixed voltage output and the resistance, which will be variable.

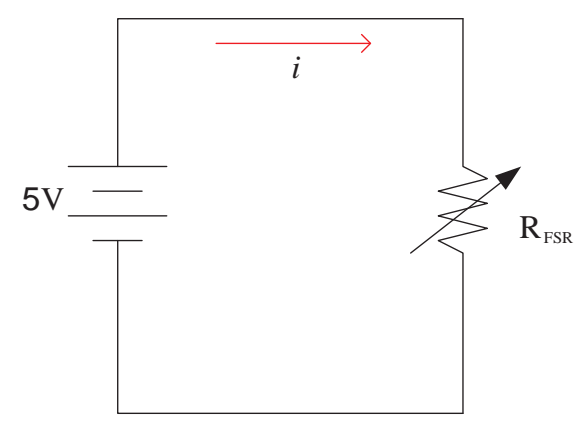

Figure 3.3 Basic circuit configuration using an FSR.

Since the range of an FSR varies from $1 \mathrm{M} \Omega$ to $0.2 \mathrm{k} \Omega$ based on experimental data, with a $5 \mathrm{~V}$ power supply, the currents will vary between $5 \mu \mathrm{A}$ to $25 \mathrm{~mA}$. Due to the output current are extremely small, the output might not be processed unless special processing circuits are adopted. For instance, a transistor can be used to amplify the current but it will also add possible disturbances and noise in the measurements. Therefore, measuring the current is not suggested for FSR sensors. 


\subsubsection{Voltage Measurement}

Clearly, current measurement is not and optimistic way to measure applied force. Thus, voltage measurements can be a better alternative way to measure an FSR. However, the circuit shown in Figure 3.3 generates identical voltage despite the changing resistance. Therefore, in order to read a varying voltage of an FSR, due to its resistive change an additional resistor $\left(R_{a d d}\right)$ needs to be added to the circuit. The Figure 3.4 illustrates the diagram of the circuit. To guarantee that the data acquisition device can always read the voltage variation according to the resistive change, an optimal value of $R_{a d d}$ needs to be determined.

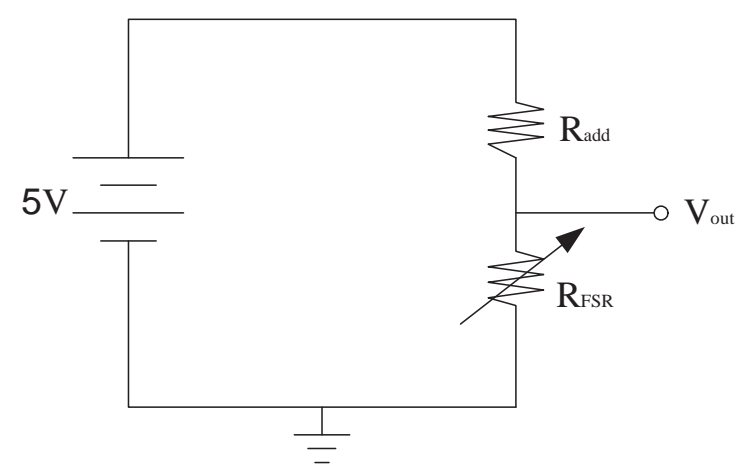

Figure 3.4 Circuit of a voltage divisor using an FSR

Equation 3.1 represents the voltage output of a typical voltage divisor.

$$
V_{O U T}=\frac{R_{F S R}}{R_{a d d}+R_{F S R}} V_{I N}
$$

Based on the experimental data the range of resistance of a single FSR varies from $5.7 \mathrm{k} \Omega$ to $0.36 \mathrm{k} \Omega$ corresponding to an applied force between 2 to $90 \mathrm{~N}$. However, a Thru mode FSR array shifts the range between $30.8 \mathrm{k} \Omega$ to $0.33 \mathrm{k} \Omega$ for the same force interval. 
Considering these values and applying equation 3.1, the output voltage can be determined. Table 3.1 shows the ranges of output voltage for different resistor connected to a single FSR. It is clear that extremely large or small $R_{a d d}$ yields a small readable range of resistance.

\begin{tabular}{|c|c|c|c|}
\hline \hline Resistance $(\mathrm{k} \Omega)$ & Max. Voltage(V) & Min. Voltage(V) & Range Modulus(V) \\
\hline \hline 0.001 & 4.999 & 4.986 & 0.013 \\
0.01 & 4.991 & 4.865 & 0.126 \\
10 & 1.815 & 0.174 & 1.642 \\
100 & 0.270 & 0.018 & 0.252 \\
1000 & 0.028 & 0.002 & 0.027 \\
\hline
\end{tabular}

Table 3.1 Range of voltages using different additional resistors connected to a single FSR.

Based on Table 3.1 the best range is suggested when the additional resistance is $10 \mathrm{k} \Omega$ for a single FSR. On the other hand, for a Thru Mode array FSR the results are slightly different, as shown in Table 3.2. The range is really narrow for extremely small or large values of resistors. With a resistor of $10 \mathrm{k} \Omega$, the modulus range is $3.6 \mathrm{~V}$. Thus, the add on resistor is set to be $10 \mathrm{k} \Omega$ for the FSR array.

\begin{tabular}{|c|c|c|c|}
\hline \hline Resistance $(\mathrm{k} \Omega)$ & Max. Voltage $(\mathrm{V})$ & Min. Voltage(V) & Range Modulus(V) \\
\hline \hline 0.001 & 5.000 & 4.985 & 0.015 \\
0.01 & 4.998 & 4.853 & 0.145 \\
10 & 3.775 & 0.160 & 3.615 \\
100 & 1.177 & 0.016 & 1.161 \\
1000 & 0.149 & 0.002 & 0.148 \\
\hline
\end{tabular}

Table 3.2 Range of voltages using different additional resistors connected to a Thru mode FSR array. 


\subsection{Advantages and Disadvantages of FSR}

The major advantages of FSR include: 1) the supporting circuit is easy to implement and 2) the cost is cheap compared to other types of sensors. However, the repeatability and accuracy of such kind of sensors are poor. For instance, identical force with same magnitude may not always obtain the same resistance reading. Such a phenomenon is extremely severe for a force between 0 to $20 \mathrm{~N}$. Thus, precision of these kind of sensors need to be improved hardware-wise. In this study, an approach regarding to the implementation of super resolution algorithm which can increase the resolution of sensor array was investigated. The procedure and the performance will be discussed in the next chapters.

\subsection{Experimental Platform}

\subsubsection{Experimental Setup}

An experimental setup was established. In order to identify the relationship between the applied force and resistance to validate the repeatibility of the adopted sensor. A single FSR and the Thru mode FSR array manufactured by Sensitronics were used to perform this experiment. The experimental procedure starts from applying a range of forces between 0 to $90 \mathrm{~N}$ by a force gauge. Once the force is applied, the resistance of the sensors was measured by a multimeter. The identical force is applied to the same sensor several times, and the correspondent resistances are recorded. The experimental setup is shown in Figure 3.5, which includes an advancing slider with a driving wheel, a force gauge Shimpo FGE-20X and a sensor platform. As the force tip touches the sensor, the applied force can be controlled by rotating the driving wheel to advanced or withdraw the slider. The applied force is measured and visible via the force gauge. 


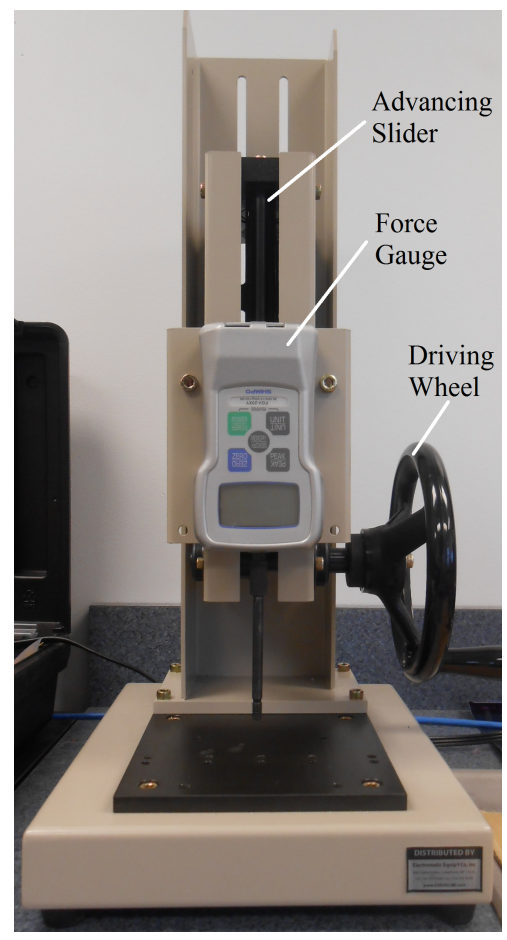

Figure 3.5 Experimental setup of the force measurement system.

\subsubsection{Force Measurements of a Single FSR}

In the case of a single FSR sensor, the first step is to connect the FSR terminals to a regular multimeter. Then the sensor is placed on the platform and the force is applied to the sensor through the force gauge controlled by the driving wheel located aside the platform. Figure 3.6(a) demonstrates the real measurement setup. In this study, one single FSR sensor was tested twelve times for consistence purposes. For each sensor, 17 forces with different magnitudes were applied to establish the possible mathematical model. In order to accurately measure applied force, some criteria needs to be considered. First of all, the gauge tip needs to be centered in the middle of the single FSR. Otherwise, the resistance values can vary considerably. Figure 3.6(b) shows the correct way to place the gauge tip on of sensors. The change rate of applied force needs to be limited as well. The change rate of applied force has to be small until the desired force is reached. To get accurately readings, the applied force cannot exceed the desired force. If the applied force is greater that the 


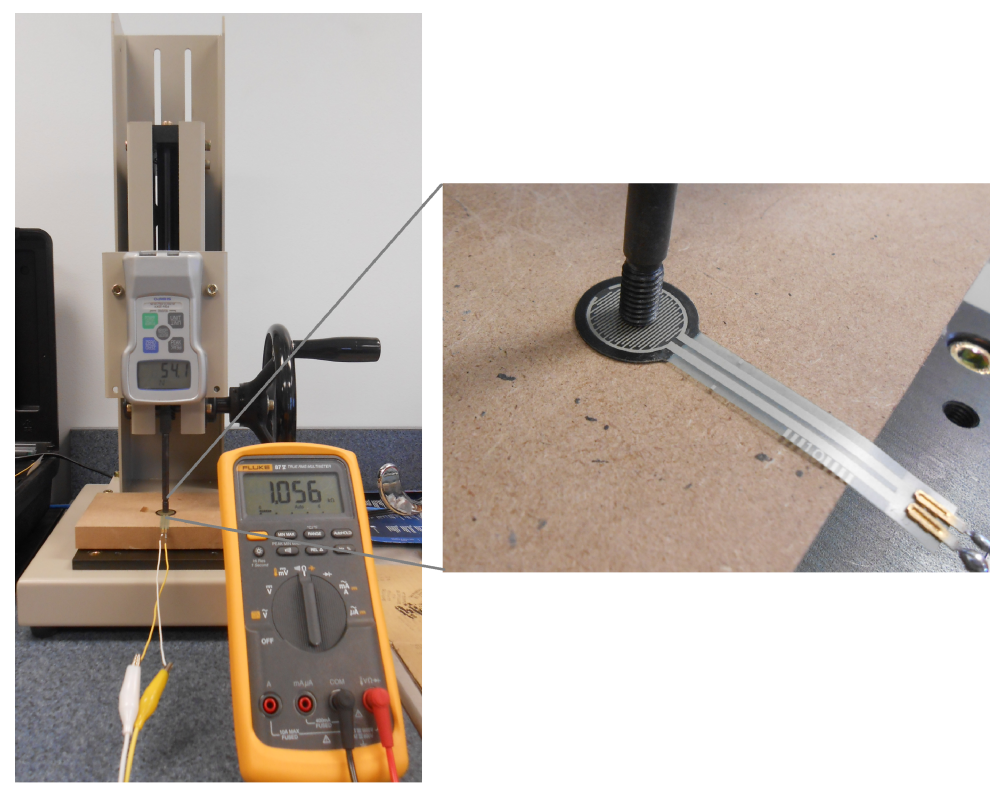

(a)

(b)

Figure 3.6 a) Experimental setup that is used to measure the resistance of a single FSR caused by the applied force. b) Correct location to place the gauge tip on the FSR

desired value, the measured value can be greatly affected. As to the settling time of the FSR sensor, it is suggested to wait for at least 5 seconds before a new measurement is taken.

\subsubsection{Force Measurements of a Thru mode FSR array}

To identify the relationship between applied force and measured output of a Thru mode FSR array, the procedure is similar to the one used for a single FSR. However, since the array has 160 sensors, it is not realistic to identify all the 160 relationships. Instead, 22 sensors in 6 different areas were tested. Figure 3.7(a) shows the experimental setup. Figure 3.7(b) illustrates the diagram of the sensor array and the locations of the selected sensors. Similar to the procedure of a single FSR measurement, a force between 0 to $90 \mathrm{~N}$ was applied. 17 forces with different magnitude were applied and the resistance data was recorded manually. All registered values and the statistical analysis of them are shown in the following section. The criteria mentioned in the previous section also applied to the 


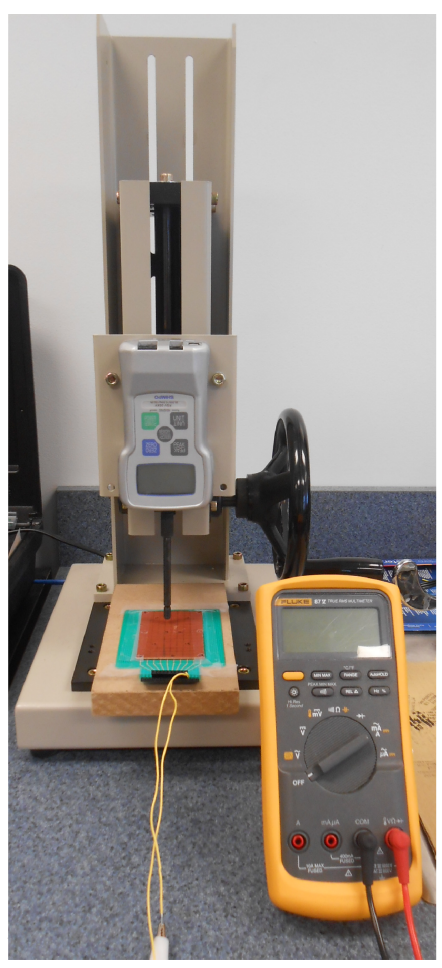

(a)

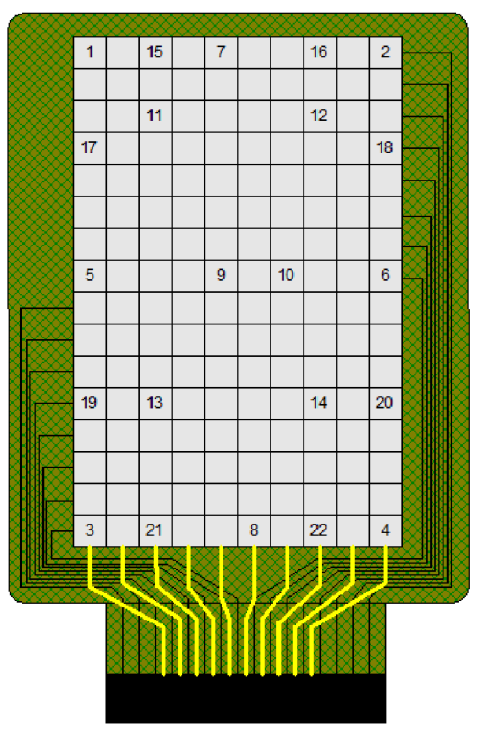

(b)

Figure 3.7 a)Experimental Setup that is used to measure the resistance of a Thru Mode array caused by applied force. b) Locations of the measured sensors.

measurement of an array. The gauge tip has to be placed at the middle of each sensors, and the change rate of loading needs to be considerable to have effective measurements.

\subsubsection{Measurements and Data Collection}

\subsubsection{Circuit Design of Sensor Array}

In order to efficiently acquire readings of individual sensors of the Thru mode FSR array, a supporting circuit that converts force to voltage was fabricated. Figure 3.8 shows a diagram of the design circuit. The circuit is capable to read all 160 sensors of the Thru Mode FSR array. The circuit integrates two multiplexers, 74HC4067E, which can control up to 18 channels. The purpose of the use of these devices is to simplify the 160 individual 


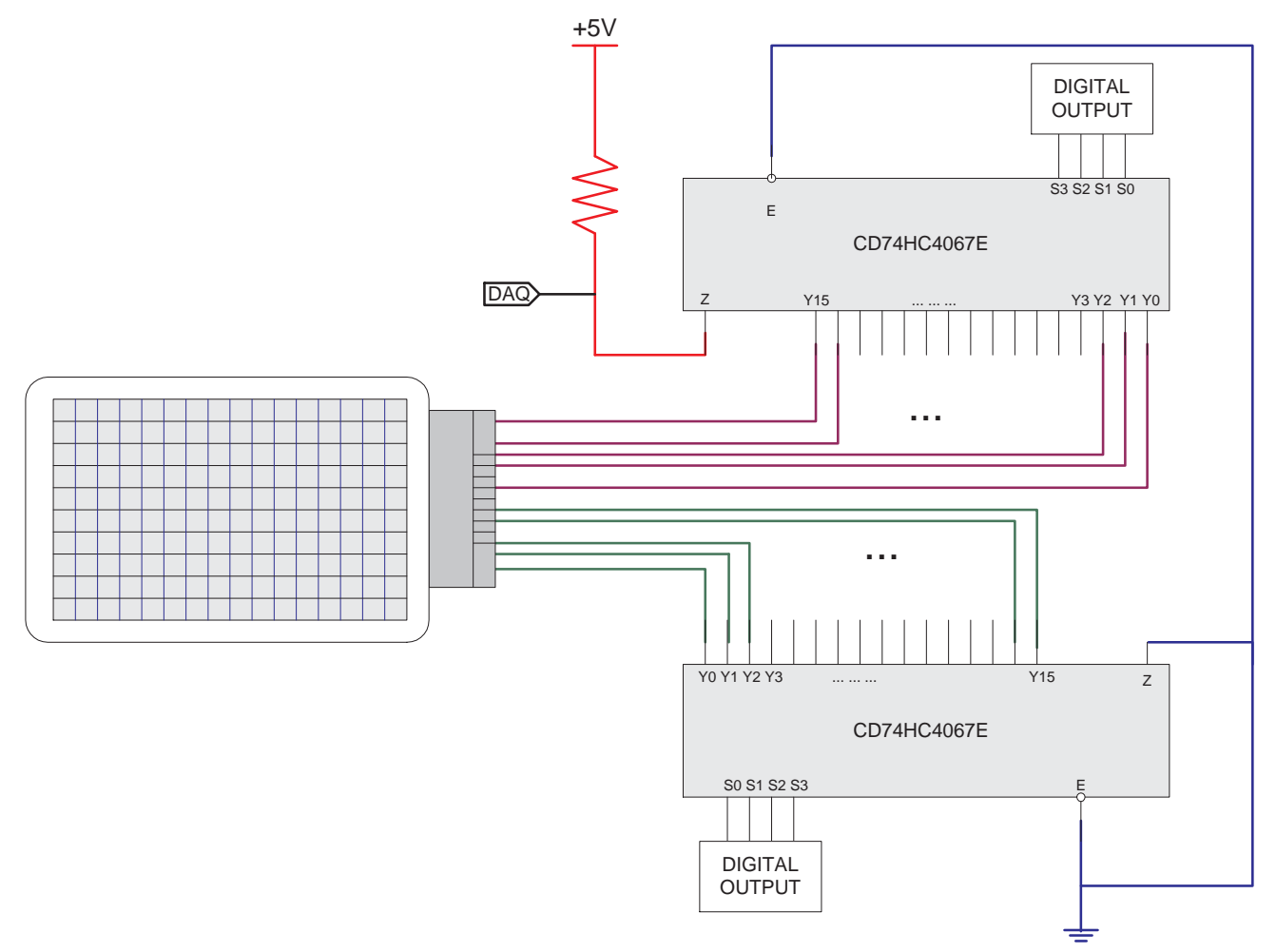

Figure 3.8 Schematic diagram of the implemented circuit to read the resistance generated by a Thru mode FSR array

voltage divider circuit. One multiplexer is in charge of selecting the row and the other one is in charge of selecting the column. The states of the multiplexers are controlled by four digital inputs. The digital outputs are designated by a data adquisition (DAQ) device. A add on resistor of $10 \mathrm{k} \Omega$ is part of the voltage divider and the input voltage is set to be $5 \mathrm{~V}$. The output voltage can be acquired by the analog input of the DAQ device. The outputs and inputs are processed by a MATLAB script. Once the circuit gets the value of the output voltage. In order to converter it back to resistance, Equation 3.1 can be rewritten as,

$$
R_{F S R}=\frac{10000 \Omega * V_{O U T}}{5-V_{O U T}}
$$




\subsubsection{Data Acquisition Station}

The communication between the external circuit and computer was implemented with a data acquisition target and a MATLAB script that manages the analog input and digital outputs. Other than data acquisition the script is also in charge of converting the acquired voltage to force and displaying the image of the geometric shape of the object placed on the surface of FSR array. The data acquisition board used for this research is NI-PCI-6221

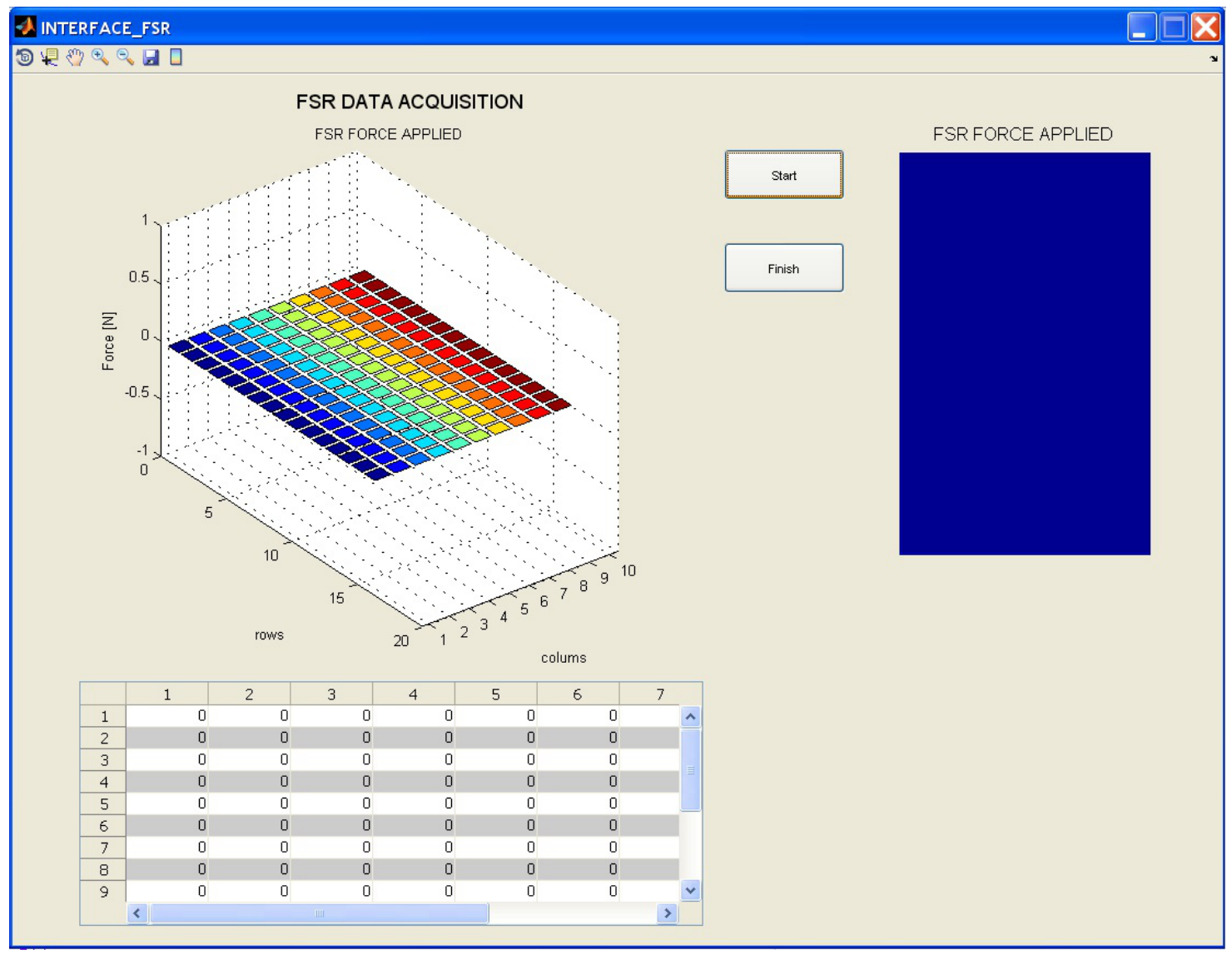

Figure 3.9 Graphic-user-interface of the developed MATLAB script

DAQ. The MATLAB script also has a Graphic-User-Interface (GUI) which includes three main parts: a three dimensional block display of force distribution for every sensor, a table 
$16 \times 10$ that demonstrates the numerical readings of applied force to the array, and a two dimensional image $(16 \times 10$ pixels $)$ that shows the preliminary shape of the object placed on the array. The interface is shown as Figure 3.9. The estimated time to perform a complete reading of all 160 sensors is approximately 18 seconds. Figure 3.10 shows an example of the DAQ system working as a whole, including the circuit, the DAQ device, interface and the Thru mode FSR array.

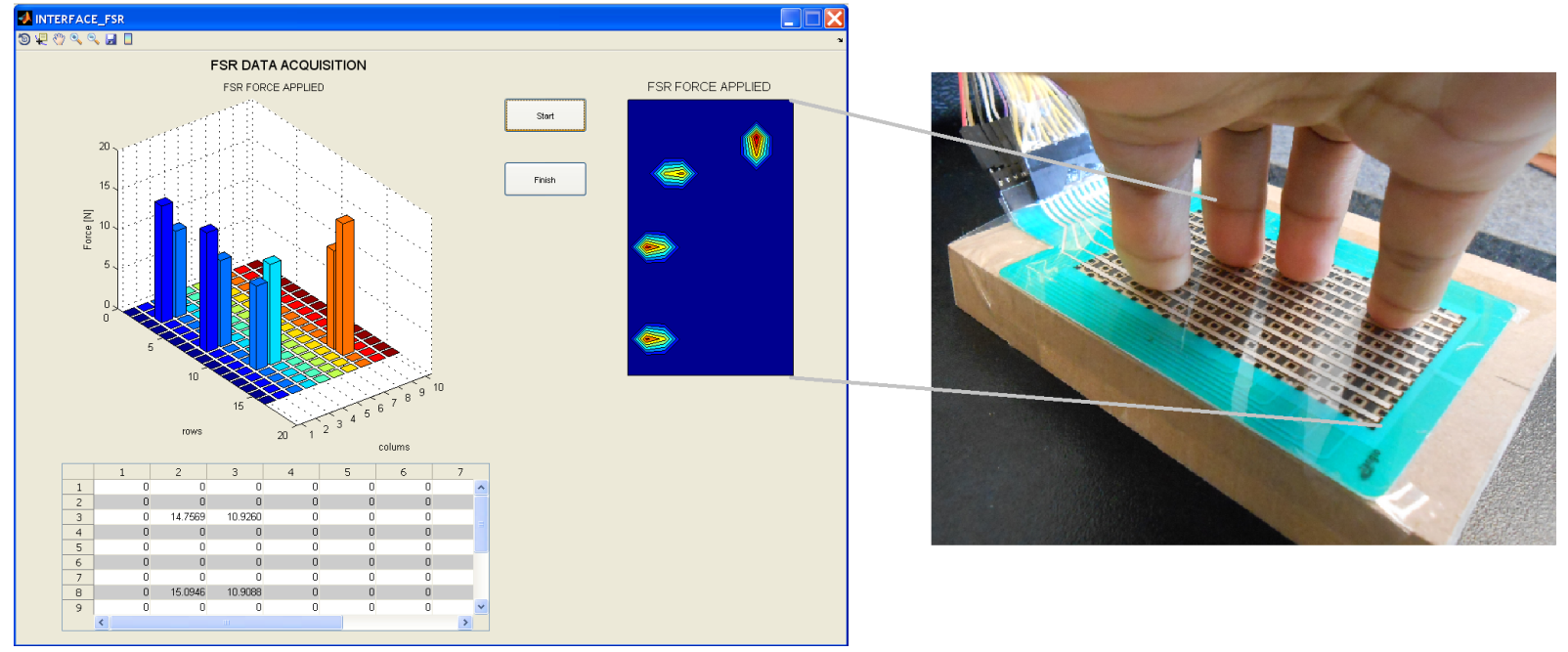

(a)

(b)

Figure 3.10 Experimental measurement using DAQ and the GUI: a) Display of measured outcome with GUI, b) physical positions of applied forces

\subsection{Statistical Analysis of the Measurements}

\subsubsection{Collected Data from a Single FSR}

Due to the manufacturing process, it is anticipated that the resistive outputs of the sensor approximates to normal distribution. With this assumption, the data acquired from the experiments was modeled by statistical analysis. Thus, the mean value $(\bar{x})$ and the 
standard deviation $(\sigma)$ are the two important parameters that need to be computed. The standard deviation can be obtained by the following Equation 3.3 which is:

$$
\sigma=\sqrt{\frac{1}{N} \sum_{i=1}^{N}\left(x_{i}-\bar{x}\right)^{2}}
$$

where $N$ is the total population and $\bar{x}$ represents the mean value. For a single FSR sensor twelve set of measurements were taken and forces with 17 different magnitudes were applied. In total, 204 measurements were collected. Figure 3.11 shows the graph of the mean and the $\pm 3 \sigma$ plots, which covers $99.73 \%$ of probability of the force output of the same kind of sensors.

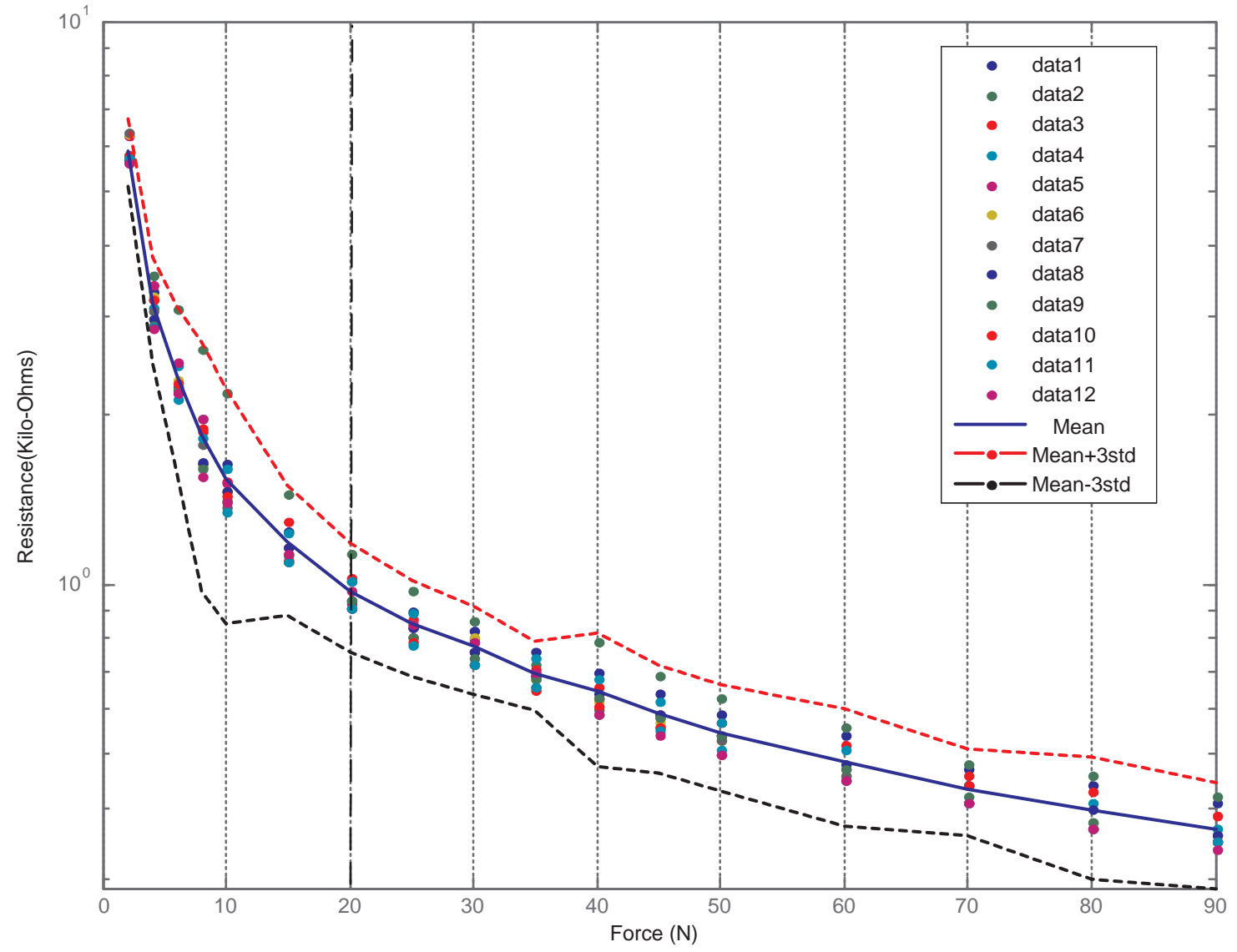

Figure 3.11 Collected data from a single FSR and the statistical model. 


\subsubsection{Collected Data from the FSR Array}

The same statistical analysis for a single FSR was applied to the experimental data of the Thru mode FSR array. In this case, seventeen values of forces were applied to 22 sensors dispersed on the surface of the array. The total amount of measurements taken were 374. Once the data was collected the mean value and the standard deviation were computed. Figure 3.12 shows the distribution of the mean $\pm 3 \sigma$ graph.

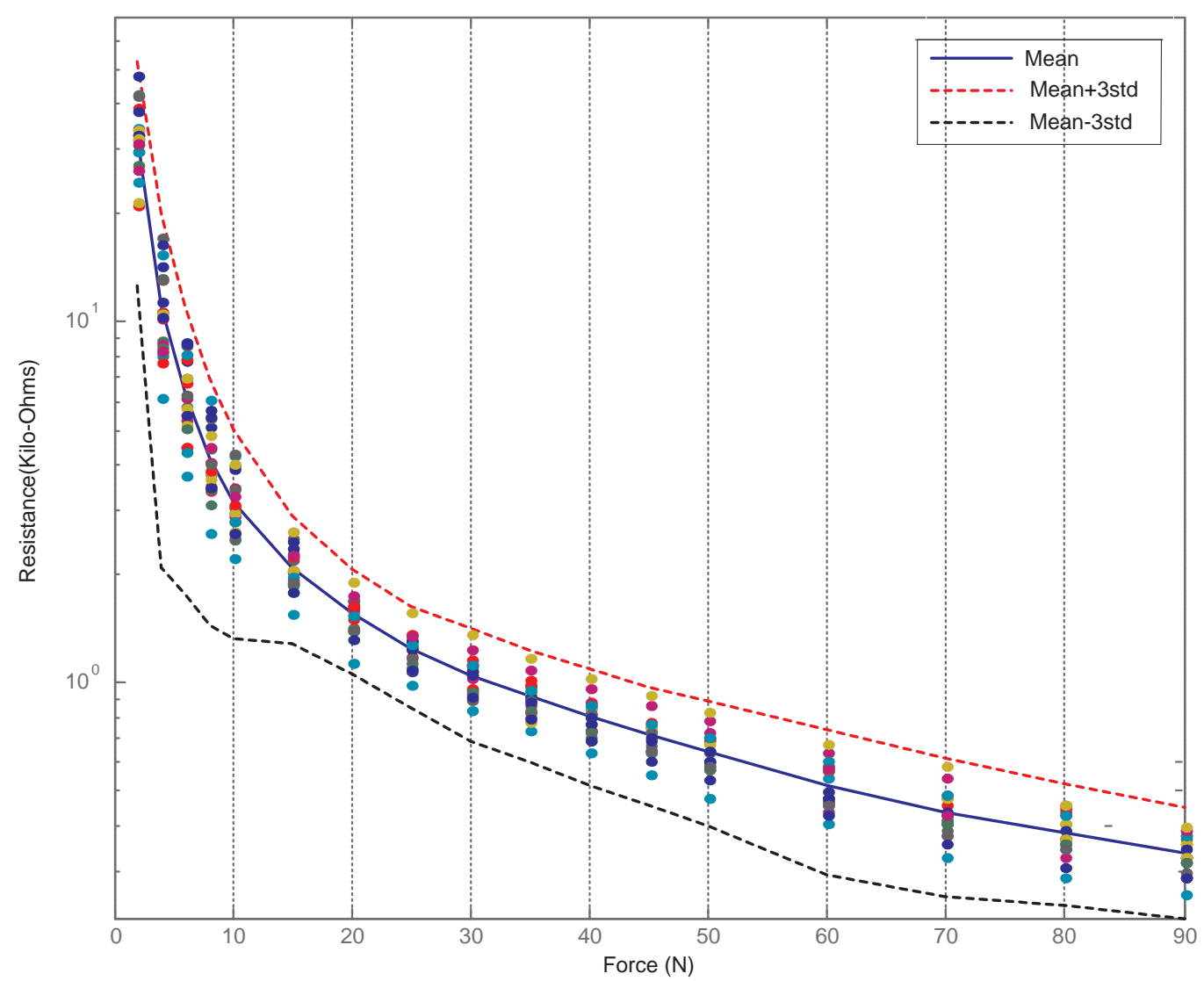

Figure 3.12 Collected data from a Thru mode FSR in terms of mean, and $+/-3 \sigma$. 
After analyzing the data, two facts need to be highlighted. Lower-limit of applicable force: It is clear that the variation or resistance is increased dramatically when the force is smaller than 20 Newtons. For instance, a force of $10 \mathrm{~N}$ can yield the resistance change from $2.22 \mathrm{k} \Omega$ to $4.27 \mathrm{k} \Omega$, which doubles the value easily. With forces greater than 20 Newtons the measured data becomes much more consistent and reliable. The resistances demonstrates smaller standard deviation. As a result, it can be concluded that the sensors can work as two modes, the ON/OFF mode and the lecture mode. In the ON/OFF mode, the sensor can be used only to detect whether or not a force is applied when the force is smaller than $20 \mathrm{~N}$. The second mode is lecture mode, where the value of the force can be read accurately if it is greater than $20 \mathrm{~N}$.

\subsubsection{Location-Oriented Model}

Since the selected sensors of the array were distributed at the corners, central area and border lines, an interesting pattern was discovered. From the measured readings, the sensors can be grouped into several categories for better approximation of statistical models at specific locations. For example, the sensors located along the border have similar responses between applied force and resistance.

As a result, depending on the location, the sensors behave differently. This can be explained due to manufacturing defects, stress caused by fabrication can generate preexisting force on the FSR sensor before external force is applied. Moreover, because of some fabrication processes the original distance between the substrates and spacer is not the same for all the 160 sensors. Hence, after analyzing the experimental data all the tested points were classified in six different groups. Figure 3.13 shows a group map of the Thru Mode FSR array. 


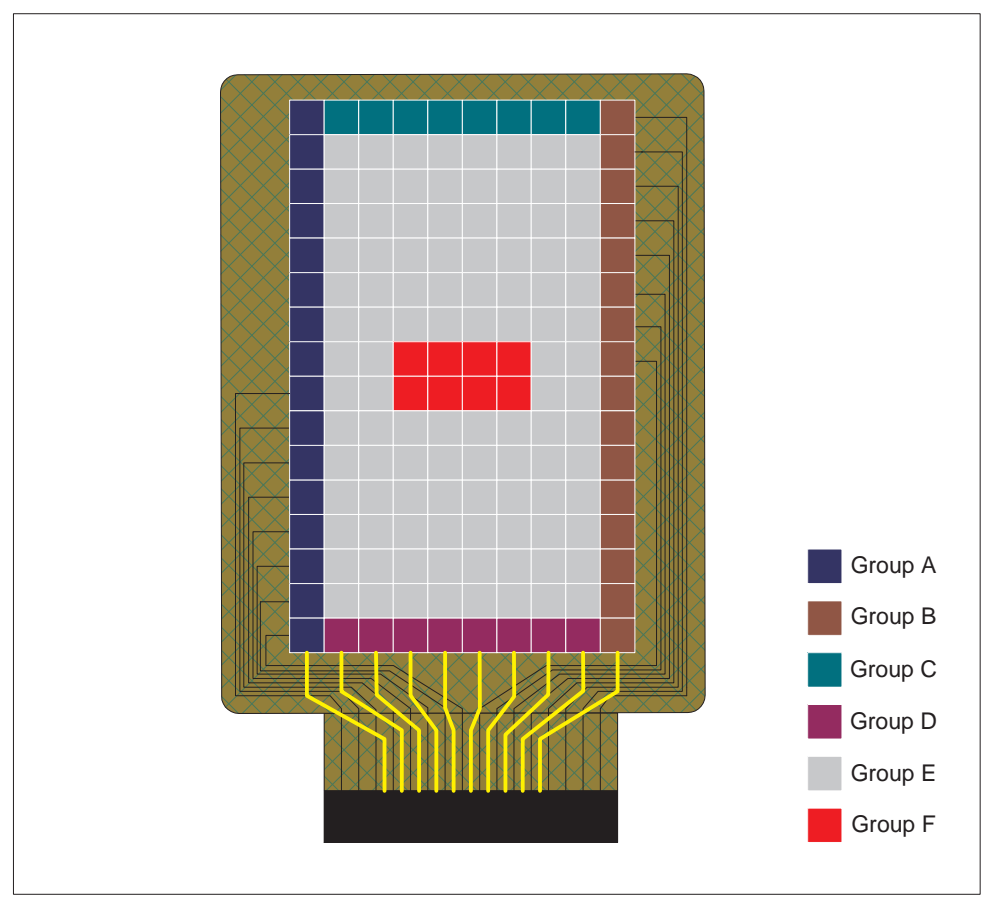

Figure 3.13 Group map of a Thru Mode FSR array

\subsection{Modeling Results}

As stated in previous sections, 160 sensors of a Thru mode FSR array are divided into six groups. Figure 3.14 illustrates statistical distribution of each group. In those graphs, the solid line is the mean value of the measurements and the dash lines are the border line of $\pm 3 \sigma$. As expected, all the graphs indicate the response between the resistance and the force become consistent if the applied force is greater than $20 \mathrm{~N}$. Moreover, separating the sensors into different groups also yields smaller standard deviations. 

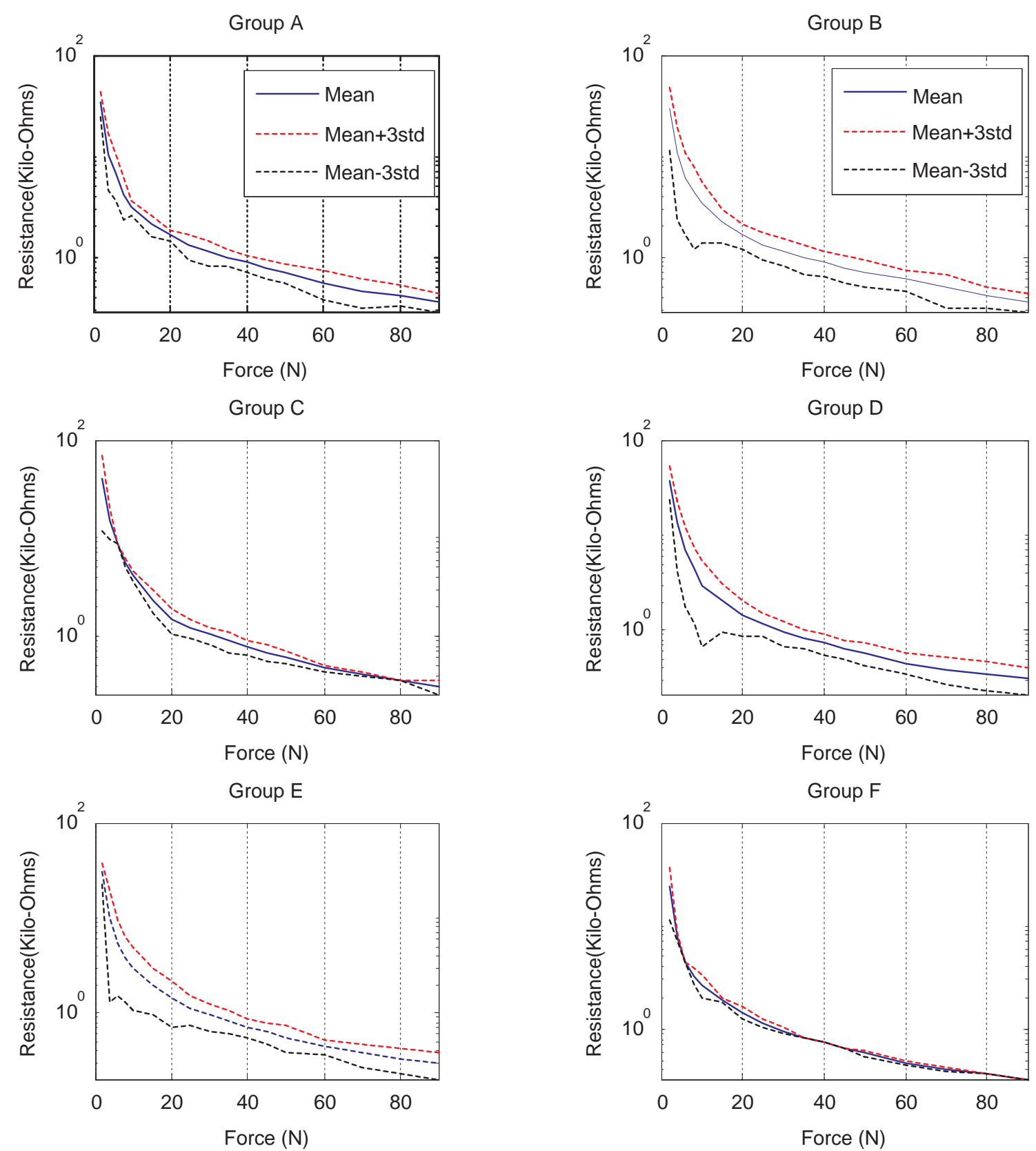

Figure 3.14 Plots for every group of a Thru mode FSR array.

In Figure 3.15 the mean values of all 6 groups are compared. It is clear that the behavior of every group is similar with some minor shifts along y axis. However, in case that some precise experiments need to be performed using a Thru Mode FSR array, individual 
graphs need to be taken into account.

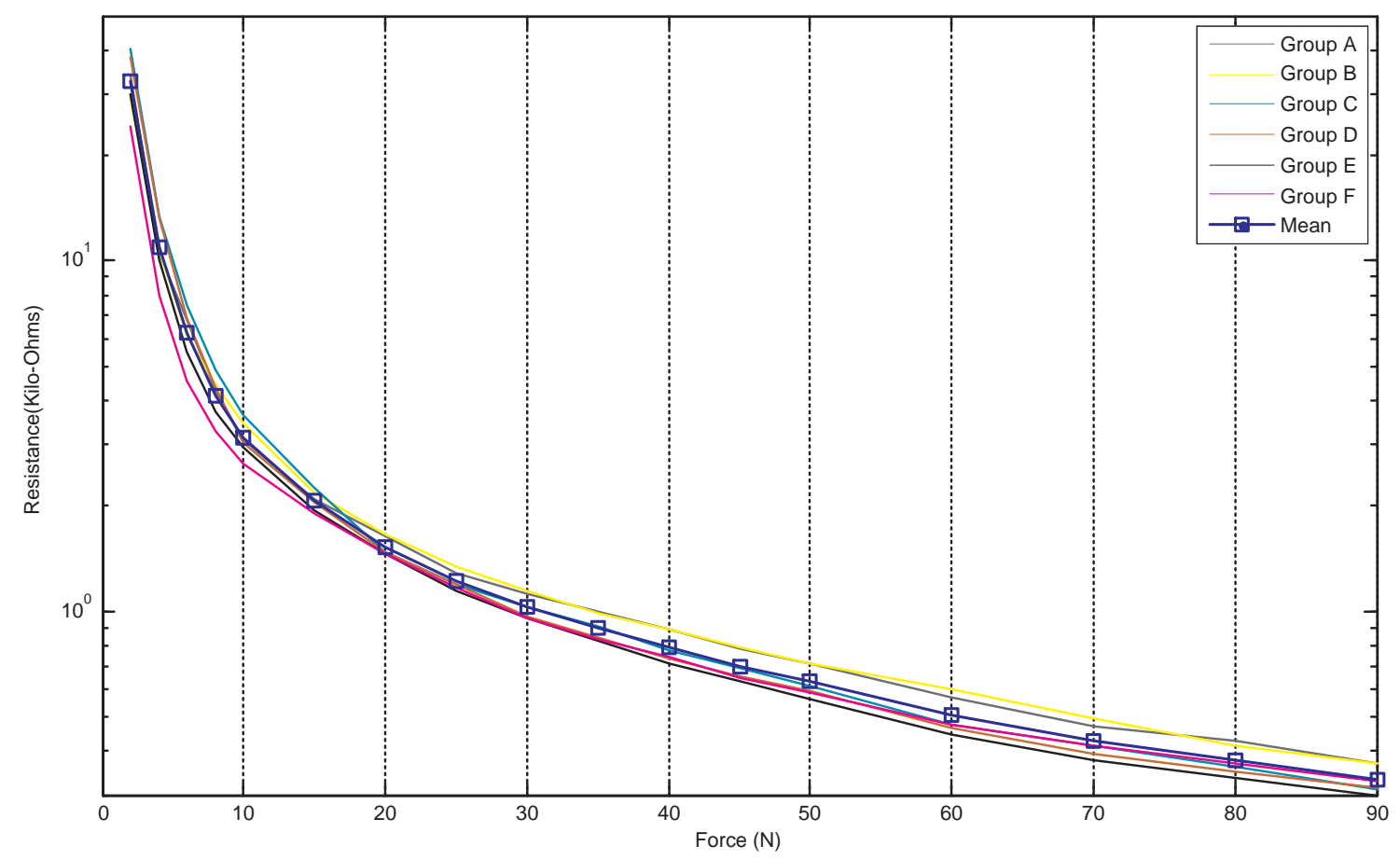

Figure 3.15 Plot of the mean of the six groups, also the graph for each group is included.

Based on the experimental results, Table 3.3 shows the statistical models of all six sections, where $\mathrm{F}$ is the force and $\mathrm{R}$ is the resistance of the FSR. These equations are then implemented in the MATLAB script to be to converted from resistance to force accurately. When a Thru mode FSR array is used in a engineering application where the major concern is the precision, it is suggested to adopt individual statistical models for each group. In case precision is not so important, the statistical model of the mean of all groups will provide reliable results. 


\begin{tabular}{|c|c|}
\hline \hline Groups & Equation \\
\hline \hline $\mathrm{A}$ & $R=49.936 F^{-1.105}$ \\
\hline $\mathrm{B}$ & $R=47.183 F^{-1.086}$ \\
\hline $\mathrm{C}$ & $R=80.022 F^{-1.259}$ \\
\hline $\mathrm{D}$ & $R=63.284 F^{-1.210}$ \\
\hline $\mathrm{E}$ & $R=48.280 F^{-1.148}$ \\
\hline $\mathrm{F}$ & $R=34.936 F^{-1.052}$ \\
\hline Mean & $R=51.765 F^{-1.141}$ \\
\hline
\end{tabular}

Table 3.3 Mathematical model of each group of a Thru mode FSR array.

\subsection{Model Validation}

The model validation consisted in performing another set of experiments on a different Thru mode FSR array. In the experiment, a single sensor was selected from each group and a theoretical value of resistance was predicted using the corresponding models. Once the predicted values were determined, the next step was to perform the real experiments on the selected sensors. A range of forces from 20 to 90 Newtons was applied by a force gauge and the resistance was measure by a multimeter. The results were collected and compared with the expected values obtained from the models. The set of tables 3.4 show the model validation measurements between the modeled resistance and the physical measurement. As in the tables, the maximum error is $4 \%$ and the percentage average error for all the groups is approximately $0.8 \%$. Negative errors mean that the physical measurement is smaller than the modeled resistance. As a conclusion, the mathematical model is reliable to accurately predict the value of the resistance caused by the applied force. 


\begin{tabular}{|c|c|c|c|}
\hline \hline \multicolumn{4}{|c|}{ Group A } \\
\hline \hline Force [N] & $\begin{array}{c}\text { Modeled } \\
\text { Resistance }[\mathrm{k} \Omega]\end{array}$ & $\begin{array}{c}\text { Physical } \\
\text { Measurement }[\mathrm{k} \Omega]\end{array}$ & Error \% \\
\hline 20 & 1.82 & 1.83 & 0.39 \\
30 & 1.16 & 1.19 & 2.13 \\
40 & 0.85 & 0.88 & 3.69 \\
50 & 0.66 & 0.69 & 4.02 \\
60 & 0.54 & 0.56 & 3.31 \\
70 & 0.46 & 0.47 & 2.84 \\
80 & 0.39 & 0.41 & 3.90 \\
90 & 0.35 & 0.36 & 3.91 \\
\hline \hline
\end{tabular}

\begin{tabular}{|c|c|c|c|}
\hline \hline \multicolumn{4}{|c|}{ Group B } \\
\hline \hline Force [N] & $\begin{array}{c}\text { Modeled } \\
\text { Resistance }[\mathrm{k} \Omega]\end{array}$ & $\begin{array}{c}\text { Physical } \\
\text { Measurement }[\mathrm{k} \Omega]\end{array}$ & Error \% \\
\hline 20 & 1.82 & 1.85 & 1.44 \\
30 & 1.17 & 1.15 & -2.08 \\
40 & 0.86 & 0.83 & -3.48 \\
50 & 0.67 & 0.69 & 2.31 \\
60 & 0.55 & 0.56 & 1.25 \\
70 & 0.47 & 0.48 & 2.55 \\
80 & 0.40 & 0.39 & -3.74 \\
90 & 0.36 & 0.35 & -1.72 \\
\hline \hline
\end{tabular}

\begin{tabular}{|c|c|c|c|}
\hline \hline \multicolumn{4}{|c|}{ Group C } \\
\hline \hline Force $[\mathrm{N}]$ & $\begin{array}{c}\text { Modeled } \\
\text { Resistance }[\mathrm{k} \Omega]\end{array}$ & $\begin{array}{c}\text { Physical } \\
\text { Measurement }[\mathrm{k} \Omega]\end{array}$ & Error \% \\
\hline 20 & 1.84 & 1.87 & 1.51 \\
30 & 1.11 & 1.10 & -0.49 \\
40 & 0.77 & 0.80 & 3.81 \\
50 & 0.58 & 0.59 & 1.52 \\
60 & 0.46 & 0.48 & 3.78 \\
70 & 0.38 & 0.39 & 2.46 \\
80 & 0.32 & 0.33 & 2.57 \\
90 & 0.28 & 0.27 & -2.67 \\
\hline \hline
\end{tabular}




\begin{tabular}{|c|c|c|c|}
\hline \hline \multicolumn{4}{|c|}{ Group D } \\
\hline \hline Force $[\mathrm{N}]$ & $\begin{array}{c}\text { Modeled } \\
\text { Resistance }[\mathrm{k} \Omega]\end{array}$ & $\begin{array}{c}\text { Physical } \\
\text { Measurement }[\mathrm{k} \Omega]\end{array}$ & Error \% \\
\hline 20 & 1.69 & 1.66 & -1.61 \\
30 & 1.03 & 1.07 & 3.49 \\
40 & 0.73 & 0.75 & 2.78 \\
50 & 0.56 & 0.57 & 2.35 \\
60 & 0.45 & 0.46 & 2.96 \\
70 & 0.37 & 0.36 & -2.90 \\
80 & 0.32 & 0.32 & 0.00 \\
90 & 0.27 & 0.28 & 2.39 \\
\hline \hline
\end{tabular}

\begin{tabular}{|c|c|c|c|}
\hline \hline \multicolumn{4}{|c|}{ Group E } \\
\hline \hline Force $[\mathrm{N}]$ & $\begin{array}{c}\text { Modeled } \\
\text { Resistance }[\mathrm{k} \Omega]\end{array}$ & $\begin{array}{c}\text { Physical } \\
\text { Measurement }[\mathrm{k} \Omega]\end{array}$ & Error \% \\
\hline 20 & 1.55 & 1.58 & 1.93 \\
30 & 0.97 & 0.95 & -2.40 \\
40 & 0.70 & 0.68 & -2.82 \\
50 & 0.54 & 0.56 & 3.36 \\
60 & 0.44 & 0.44 & 0.00 \\
70 & 0.37 & 0.37 & 0.00 \\
80 & 0.32 & 0.32 & 0.00 \\
90 & 0.28 & 0.28 & 0.00 \\
\hline \hline
\end{tabular}

\begin{tabular}{|c|c|c|c|}
\hline \hline \multicolumn{4}{|c|}{ Group F } \\
\hline \hline Force [N] & $\begin{array}{c}\text { Modeled } \\
\text { Resistance }[\mathrm{k} \Omega]\end{array}$ & $\begin{array}{c}\text { Physical } \\
\text { Measurement }[\mathrm{k} \Omega]\end{array}$ & Error \% \\
\hline 20 & 1.49 & 1.49 & -0.32 \\
30 & 0.98 & 0.95 & -2.71 \\
40 & 0.72 & 0.71 & -1.54 \\
50 & 0.57 & 0.58 & 1.71 \\
60 & 0.47 & 0.49 & 3.96 \\
70 & 0.40 & 0.39 & -2.60 \\
80 & 0.35 & 0.35 & 0.00 \\
90 & 0.31 & 0.30 & -2.40 \\
\hline \hline
\end{tabular}

Table 3.4 Set of tables with the validation model corresponding to each group. The error percentage is based on the difference between the modeled resistance versus the physical measurement. 


\section{CHAPTER 4}

\section{RESOLUTION ENHANCEMENT ALGORITHM}

\subsection{Resolution Enhancement}

In the process of acquiring a two-dimensional image or measurement from an acquisition device or sensor, the object could be shifted from time to time. Thus, some important information about the shape and position of the object can be lost. In particular, the data acquisition device might only be able to provide a low resolution. Moreover, the operation of data acquisition can possibly add disturbances to the measurements as well. As a result, in case a single measurement is taken, the disturbances might cause information or details missing. For this reason, it is recommended to take multiple measurements in order to improve the resolution of a single image or picture. With multiple measurements, missing details in one measurement can appear in other measurements. However, due to vibrations and many other external environmental factors, measured information can be distorted dramatically. This can be extremely severe if the measurements are taken in the range of nanometers. The process of taking a measurement by an acquisition device is shown in figure 4.1. During the acquisition, data of the targeted object can experience some disturbances such as translation, rotation and vibrational blur. Thus, the acquired two-dimensional image will include these effects with extra noise from the acquisition device. As a result, the details of the targeted object might be missing due to the low resolution of the measurements. The model of the data acquisition procedure is similar to the iterated function system (IFS) proposed by Barnsley [33].

One approach to get rid of these undesirable effects and noises is by the implementation of a super resolution algorithm which consists in the collection of multiple low resolution 


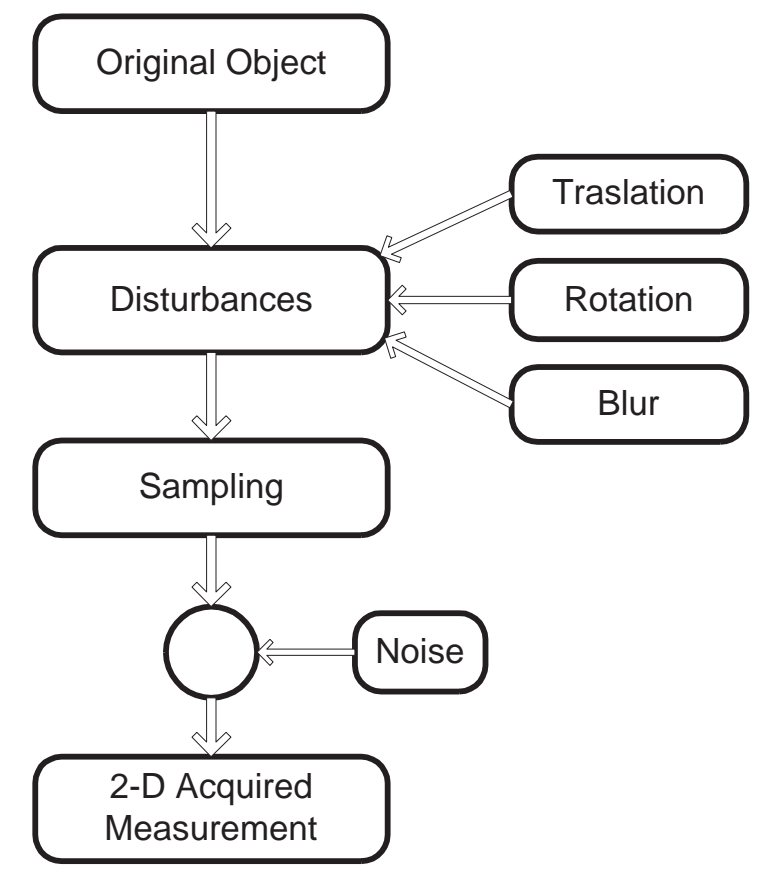

Figure 4.1 Schematic diagram of the data acquisition procedure.

images to reconstruct a higher resolution image. This methodology has been widely used for image processing in criminology, to identify a face or a license plate. It has been also applied to medical applications, to generate accurate diagnosis from tomography or magnetic resonances. Such method is also adopted by satellites to reconstruct specific geographic areas.

This chapter discusses the mathematical approach of the Proposed Resolution Enhancement Algorithm (PREA), which can be classified as a super resolution algorithm. Along this chapter, the PREA is tested by using single and multiple measurements from different sets of data acquired from distinct acquisition devices. 


\subsection{Super Resolution Approach}

The Proposed Resolution Enhancement Algorithm (PREA) or super resolution method reconstructs a high resolution image by processing several lower resolution measurements from the same object with slightly different viewpoints. In order to reconstruct a high resolution output from low resolution data, two constraints need to be modeled to solve this ill-posed problem [31]. Assume that the sampled low resolution data is $A$, which is the acquired blurred and down-sampled measurement of the targeted object, and $B$ is the high resolution output from the same scene. Thus, the relationship between $A$ and $B$ can be express as:

$$
A=S \cdot H \cdot B
$$

where $H$ is the blurring filter corresponding to a possible perturbation, and $S$ is the downsampling operator. The value of $B$ can be obtained if an adequate inverting procedure is adopted to map a low resolution sample to a high resolution output. Nevertheless, the low-resolution images can show a significant difference from sample to sample. As a result, the implementation of an inverting procedure becomes difficult to achieve. Therefore, to apply the inverting procedure, a linear interpolation among samples is adopted for a fast transformation. The proposed method includes the following phases: registration, fusion and deblurring.

In order to convert the two-dimensional acquired data from lower resolution to a higher resolution output an estimation algorithm is developed. Among different methods, the maximum likelihood approach presented by Bresler [34] is one of the most intuitive ways to define the optimal reconstructed signal $\widehat{B}$, which satisfies;

$$
\widehat{B}=\arg _{B} \min \left\{[A-S \cdot H \cdot B]^{T} W^{-1}[A-S \cdot H \cdot B]\right\}
$$

where $W$ is the weighted matrix that decreases the impact of the input noise. To obtain the maximum likelihood status of the estimated information, a gradient interpolation can 
be used. The interpolation among four adjacent samples is processed as a block. Each block is transformed from a $2 \times 2$ matrix to an $L \times L$ matrix. Hence, if the low resolution data is an image of $N \times N$ elements or pixels, the high resolution output becomes an image of dimension $L N \times L N$. The diagram in figure 4.2 illustrates the gradient interpolation applied to a block $2 \times 2$.

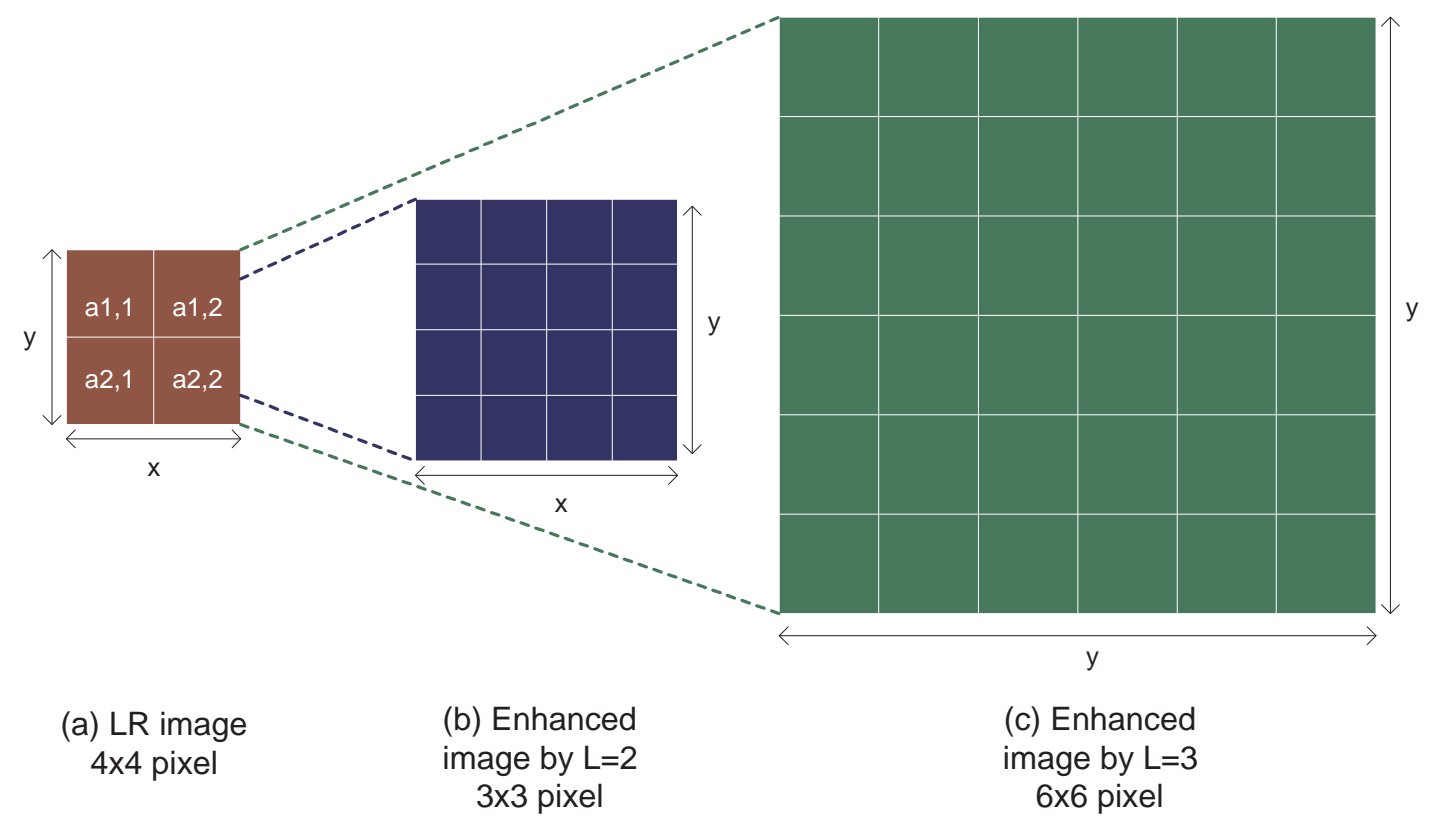

Figure 4.2 Schematic illustration of a low resolution block reconstructed to a higher resolution depending on the value $L$. The three blocks have the same high and width but the pixel density grows proportionally to the $L$ value.

Depending on the availability of data sets, the super resolution method or PREA can be applied to a single data set or multiple data sets for better image quality. The following sections demonstrate processed results of different scenarios. 


\subsection{Scenario I: Single Measurement}

\subsubsection{Resolution Improvement}

In case there is just one low resolution sample, the procedure that reconstructs a high resolution image from the low resolution measurement is based on gradient interpolation. The image is first divided into multiple blocks, the size of each block is $2 \times 2$. The gradient interpolation of the $k^{\text {th }}$ block can be represented as;

$$
\widehat{B}_{k}=[T]^{T}[A]_{k}[T]
$$

where $T$ is the gradient transformation matrix. The size of the transformation matrix $T$ is $2 \times L$, which is a linear iteration approximation matrix. The size of $A_{k}$ is $2 \times 2$, which includes the four corners of the targeted area. After the transformation the matrix $\widehat{B}_{k}$ becomes an $L \times L$ matrix. $L$ is the enhancement factor of the high resolution image. For instance if $L$ is equal to 3 the equation 4.3 can be expand as follows:

$$
B_{3 x 3}=[T]_{3 x 2}^{T}[A]_{2 x 2}[T]_{2 x 3}
$$

where,

$$
B_{3 x 3}=\left[\begin{array}{cc}
T_{1,1} & T_{2,1} \\
T_{1,2} & T_{2,2} \\
T_{1,3} & T_{2,3}
\end{array}\right]\left[\begin{array}{ll}
a_{1,1} & a_{1,2} \\
a_{2,1} & a_{2,2}
\end{array}\right]\left[\begin{array}{lll}
T_{1,1} & T_{1,2} & T_{1,3} \\
T_{2,1} & T_{2,2} & T_{2,3}
\end{array}\right]
$$

For the general case, where $L$ is equal to $n$, the equation 4.3 becomes,

$$
B_{n \times n}=[T]_{n \times 2}^{T}[A]_{2 \times 2}[T]_{2 \times n}
$$


where,

$$
B_{n x n}=\left[\begin{array}{cc}
T_{1,1} & T_{2,1} \\
T_{1,2} & T_{2,2} \\
\vdots & \vdots \\
T_{1, n} & T_{2, n}
\end{array}\right]\left[\begin{array}{cc}
a_{1,1} & a_{1,2} \\
a_{2,1} & a_{2,2}
\end{array}\right]\left[\begin{array}{cccc}
T_{1,1} & T_{1,2} & \cdots & T_{1, n} \\
T_{2,1} & T_{2,2} & \cdots & T_{2, n}
\end{array}\right]
$$

In this study, a linear gradient approximation is used. After all blocks are processed, individual blocks can be combined and the synthesized output becomes;

$$
\widehat{B}=\bigcup_{k=1}^{N x N} \widehat{B}_{k}
$$

A gradient interpolation is implemented due to its fast calculation speed. Increasing the resolution one hundred times of an image of $38 \times 34$ pixels takes about 1.025 seconds.

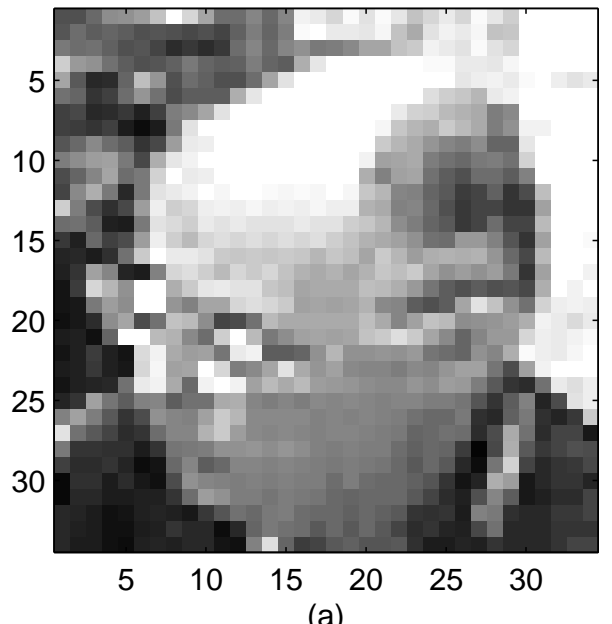

(a)

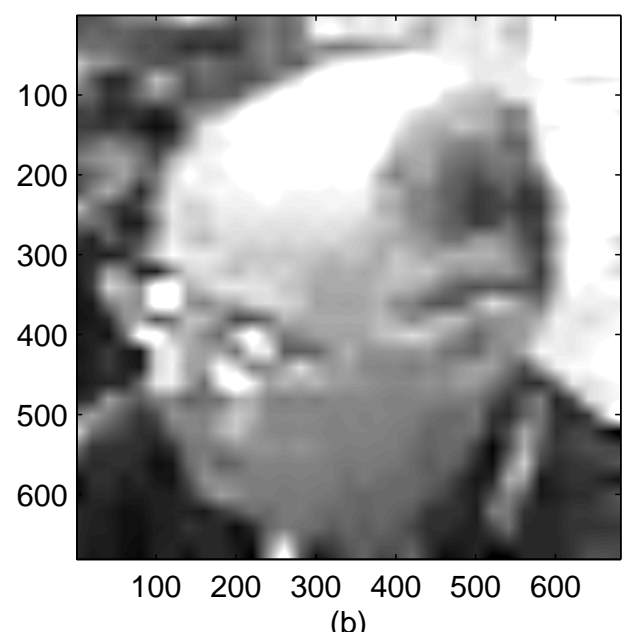

(b)

Figure 4.3 Application of the proposed resolution algorithm for surveillance purposes. a) The input data is a distorted face and b) high resolution image with a density of $681 \times 681$ pixels. The simulation time of the algorithm was 0.484 seconds. 
Figure 4.3 shows the result after applying the proposed algorithm to a low resolution image. The original image has a dimension of $38 \times 34$, which was enhanced with a factor of $L=20$. The reconstructed high resolution image shows clearer defined edges and it is possible to clearly visualize more details in the picture, such as the lenses, face contour and hair.

Figure 4.4 shows a portion of a high resolution photograph. The enhancement proposed method was applied to this image using different values of $L$ with the purpose of identifying the optimal value for $L$, the proposed results are illustrated in Figure 4.5. From these results some conclusions can be made. Though the enhanced image with a greater ratio equal to 25 shows smoother edges, it can suppress some important information, such as shadows and color intensity. As a result, after $L=10$, the density pixel increases and the edges get more defined but on the other hand some information starts missing. For these reasons, the optimal value of $L$ is 10 .

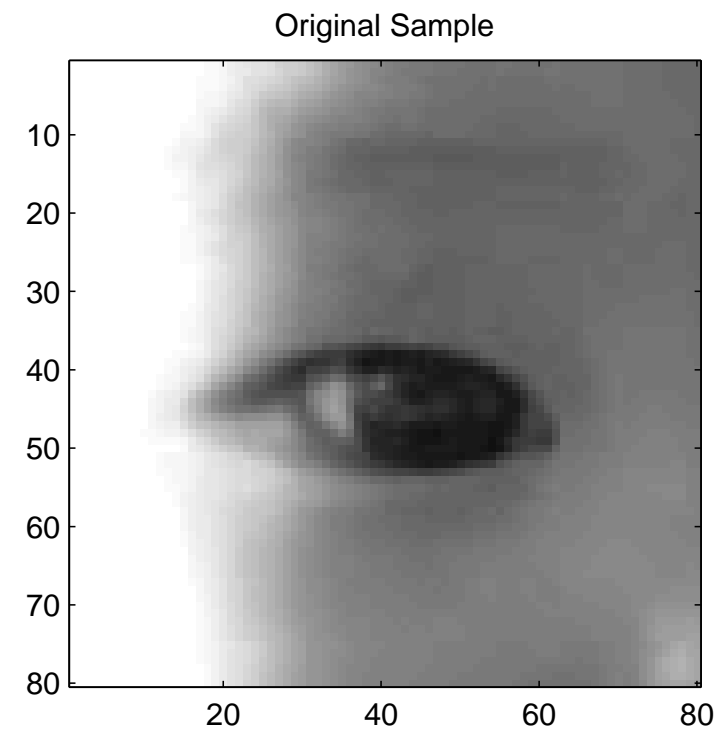

Figure 4.4 Low resolution image with a $80 \times 80$ pixel density. 

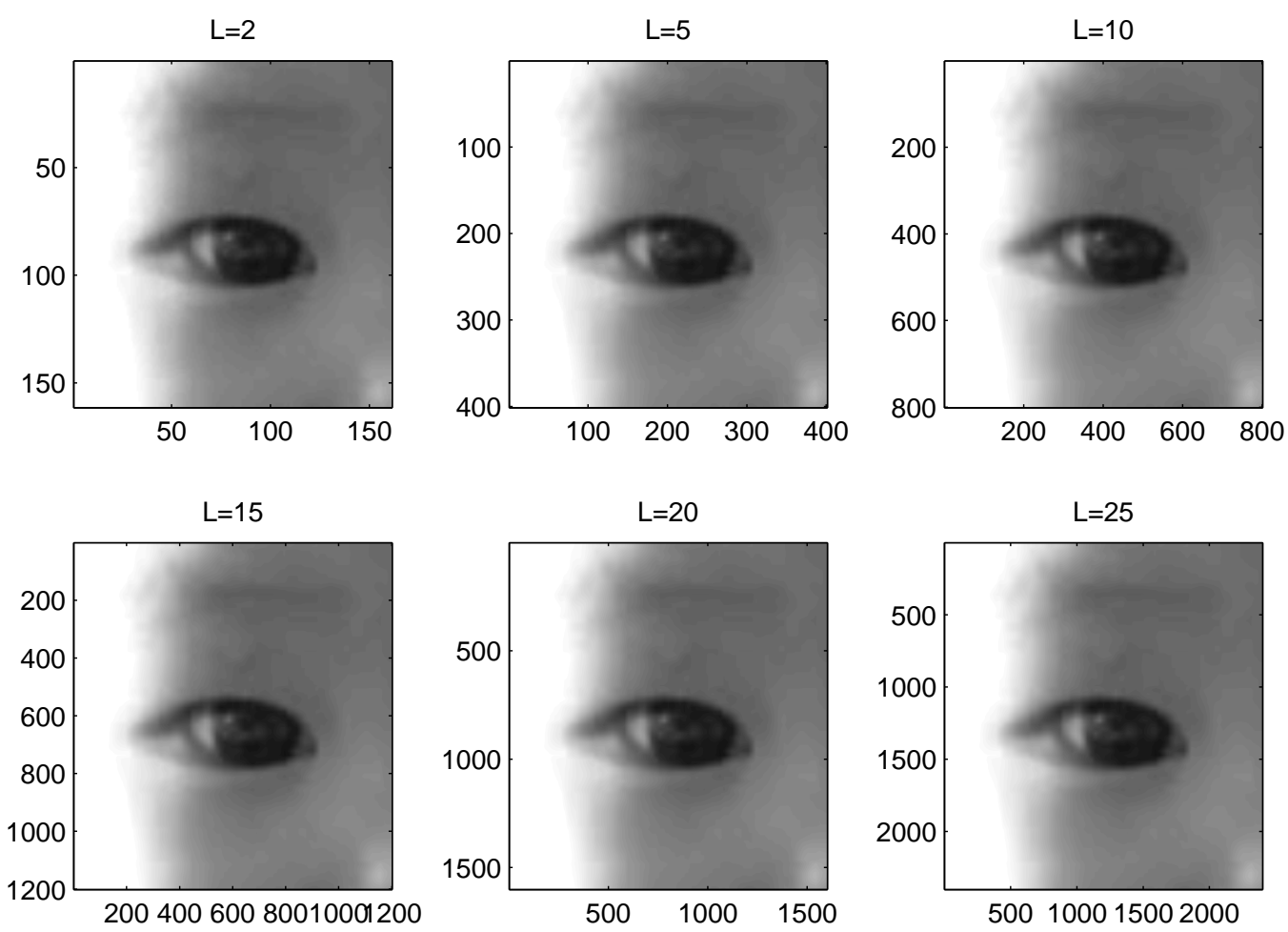

Figure 4.5 Enhancement of a low resolution image applying six different ratios $L=2$, $L=5, L=10, L=15, L=20$ and $L=30$

\subsubsection{Image Downsizing}

To evaluate the proposed resolution enhancement algorithm, it is necessary to develop a technique that can efficiently down-sample the reference data. The resolution of a known image is decreased by a factor $D$, which is an integer number. Since, the down-sampling provokes to lost some information, it is primordial to get as much information as possible after decreasing the resolution. The proposed technique to obtain the most efficient downsampled image with highest quality of the up-sampled data is similar to the one presented by Zhang et al. [35]. 
The down-sampled image should satisfy the equation:

$$
U=\arg _{U} \min \|\widehat{V}-V\|^{2}
$$

where $U$ is the downsampled image $X / D \times Y / D$, where $L$ is the compression ratio, $\widehat{V}$ is the high resolution image after the application of the proposed algorithm refered in previous sections, and $V$ is the original image $X \times Y$. Figure 4.6 shows the downsampling resolution applied to a high resolution picture $375 \times 375$ pixels using a compression factor of $25 \%$ and $10 \%$.
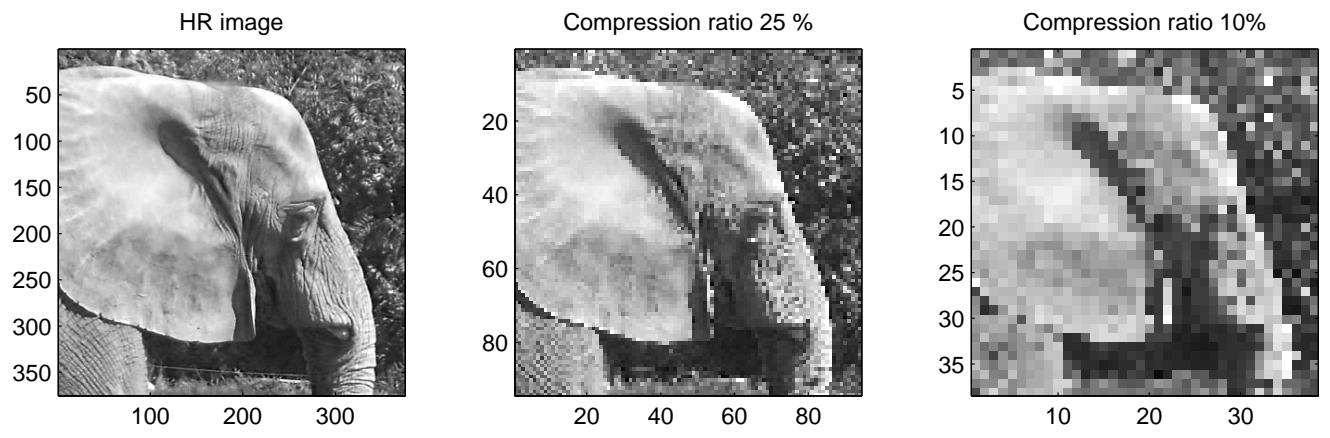

Figure 4.6 Downsampling a high resolution image using two compression ratios: $L=25 \%$ and $L=10 \%$

In Chapter 5 the downsampling technique is applied to decrease the resolution of two nanometric reference images with the purpose to reconstruct the original image by means of the computation of the proposed resolution enhancement algorithm. The reference and reconstructed image are compared by the quality image measure, peak signal-to-signal ratio (PSNR). 


\subsection{Scenario II: Multiple Measurements}

\subsubsection{Method of Object Registration}

In the previous section, the proposed method demonstrates a great performance to enhance the resolution of a single image. However, depending on the data acquisition device a single image might not be enough to identify all the details involved. Due to perturbations, user manipulation and resolution of the acquisition device, one single sample might not show all the features of the real object or scene, which means some information can be missed. As a result, the proposed algorithm can be used to increase the resolution of the sample. However, since single samples might not contain all the detail information, the enhancement method cannot identify any geometric detail. By taking multiple measurements the missing information can be possibly collected. In other words, one sample can contain some details that another sample does not have and viceversa. Therefore, it is essential to put together additional information to ensure the correctness of the data. Hence, the quality of the output image can be highly improved by applying the proposed algorithm to multiple sets of measurements and combining the final results.

In order to acquire more details, multiple measurements with minor translations and rotations in either the acquisition device or the targeted object can be helpful. In the case of measuring in micrometer or nanometer scale, the movement shift can be generated normally by environmental vibrations, thermal drifts and other disturbances. With the tiny movements, small details can be revealed. The main idea is that the acquired data

of the targeted object always has a slight difference in every measurement. By collecting these tiny differences from various sampled data sets, unclear detail of existing parts will be concentrated. Hence, it is important to develop a method to register the position of the individual sampled data sets to a common reference map and to take all the measurements to the same coordinate. 


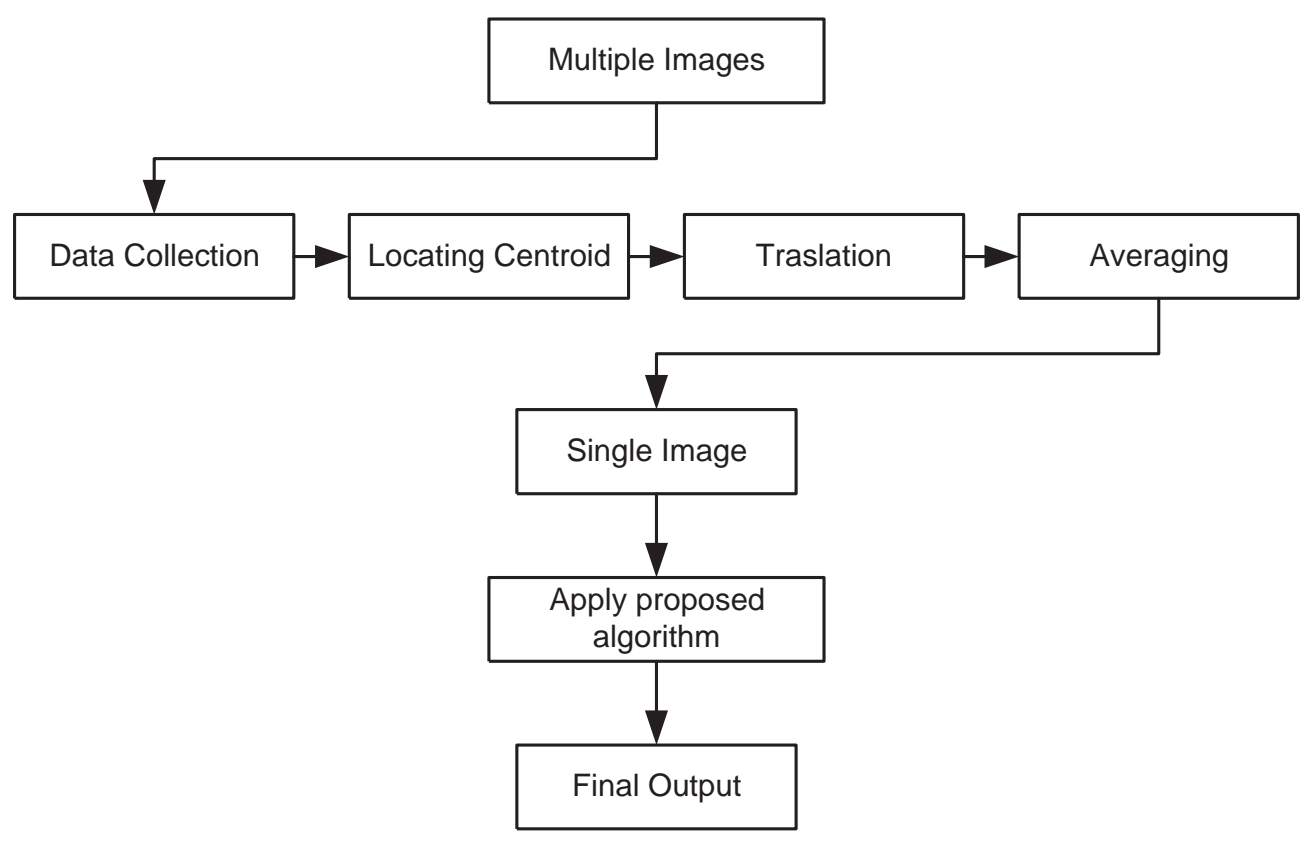

Figure 4.7 Procedure to process a set of multiple shifted images.

To find a common reference, a contrast map was adopted to identify the area occupied by the targeted object in every individual measurement. To build a contrast map, all measured samples were converted into a black and white image, which is determined by the difference among adjacent samples. If the differences are larger that a pre-set threshold, the specific locations are registered. The contrast information or local centroid of a single sample is obtained by comparing all the collected measurements. Once the contrast information is collected, the original image can be divided into clusters. All clusters with the targeted object are identified on each sample data. Thus, a common reference map can be applied to bring all the areas to the same coordinates. With all targeted objects aligned to a common coordinate, all the measurements are combined to generate a clearer output image which includes all the details of the targeted object. The best approach to combine the multiple images is by taking the mean value of all the samples. As a result, a single data set is obtained from all the measurements. The proposed gradient interpolation method is 
then applied to enhance the resolution of this single set of data. However, this procedure is just a low-pass that should be follow by a deblurring process. Figure 4.7 illustrates the block diagram that summarizes the proposed procedure to process a set of multiple images.

\subsubsection{Mathematical Approach}

Since all sampled images are slightly shifted, the implementation of a mathematical method is necessary to determine the contrast map that allows to bring all the images to the same coordinate. To simplify the calculation every image is considered as a square $N \times N$ matrix. Each element has its unique associated coordinate $(x, y)$. The targeted area is found by applying a pre-determined threshold value to individual images and locating the boundary points of the area. As a result, the maximum and minimum points in $x$ and $y$ axis of the $k^{t h}$ image can be identified. Once these values are identified, the targeted area is computed. That is,

$$
\operatorname{Area}_{k}=\left[\operatorname{Max}\left\{Y_{k}\right\}-\operatorname{Min}\left\{Y_{k}\right\}\right] \cdot\left[\operatorname{Max}\left\{X_{k}\right\}-\operatorname{Min}\left\{X_{k}\right\}\right]
$$

Though the proposed approach can locate the targeted object quickly, the length of the object in individual sampled data sets cannot be guaranteed to be the same in both $x$ and $y$ direction. Thus, it is desired determine how much individual images need to be shifted by taking the average of lengths of all samples. To define the length between the minimum and maximum point along the $x$ axis and $y$ axis, Equations 4.11 and 4.12 are implemented.

$$
\begin{gathered}
R X_{k}=\operatorname{Max}\left\{X_{k}\right\}-\operatorname{Min}\left\{X_{k}\right\} \\
R Y_{k}=\operatorname{Max}\left\{Y_{k}\right\}-\operatorname{Min}\left\{Y_{k}\right\}
\end{gathered}
$$


The mean for all minimum and maximum distances is determined by the following equations:

$$
\begin{aligned}
& M_{R X}=\sum_{k=1}^{n} \frac{R X_{k}}{n} \\
& M_{R Y}=\sum_{k=1}^{n} \frac{R Y_{k}}{n}
\end{aligned}
$$

These two values can be then used to determine the common reference of individual images, which is

$$
\begin{gathered}
\left\{X_{s}\right\}_{k}=\operatorname{Min}\left\{X_{k}\right\}+\left(R X_{k}-M_{R X}\right) \\
\left\{Y_{s}\right\}_{k}=\operatorname{Min}\left\{Y_{k}\right\}+\left(R Y_{k}-M_{R Y}\right)
\end{gathered}
$$

The images are now located in the same contrast map and can be combined together to obtain a single low resolution image that includes all the details from the set of multiple images. The following equation is applied to obtain the mean of all images.

$$
M_{\text {Img }}=\sum_{k=1}^{n} \frac{\text { TransImg }_{k}}{n}
$$

After this process, the proposed enhancement algorithm explained in previous sections is applied. Also a deblurring technique using a Wiener Filter is adopted to remove the blurred parts caused by the operation of low-pass filter. The method of registration, processing, enhancement and deblurring were implemented with a MATLAB script. 


\subsubsection{Example of Resolution Enhancement with Multiple Images}

To verify the performance of the proposed mathematical method and the enhancement algorithm. A set of ten images of a sample with indented marks was acquired. The geometric shape of the indenter tip is a Berkovich tip [36], which creates a triangular pyramid-imprint on the surface of substrate. The indented area created by the tip is less than $3 \mu \mathrm{m} \times 3 \mu \mathrm{m}$. The nine measurements were acquired by moving the indented mark in order to acquire different sights. The sizes of all images are $50 \times 50$ pixels (or $4.3 \mu \mathrm{m} \times 4.3 \mu \mathrm{m}$ ). The sampled images are shown in Figure 4.8.
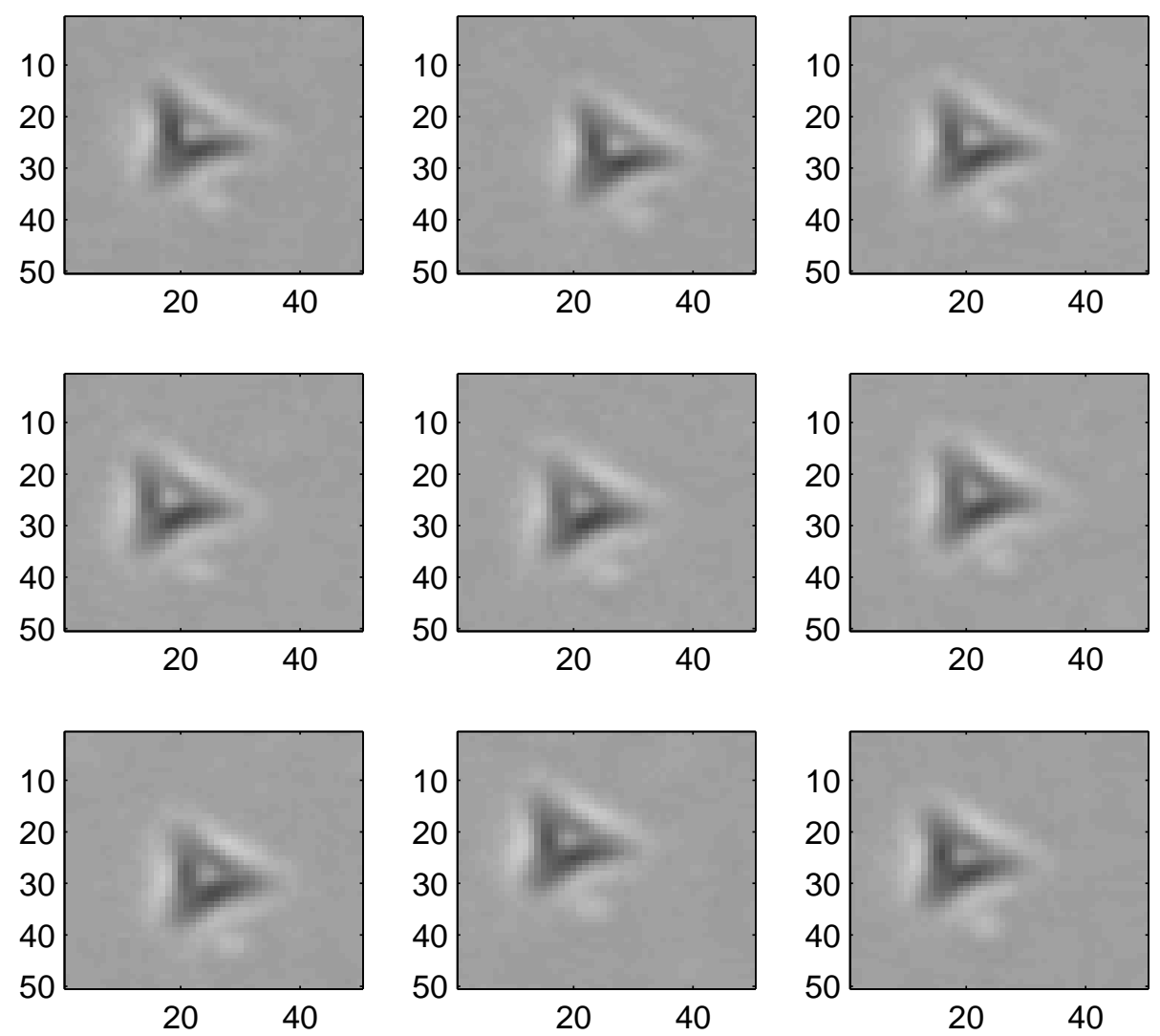

Figure 4.8 Set of nine images of a indented mark produced by a Berkovich tip. 
It is noticeable that the collected measurements show some tiny geometric differences among each other, even though the targeted object is the same. In particular, it is clear that an identical measured result cannot be repeated even if the position of the targeted object does not move at all. Thus, to calibrate the output, it is necessary to take multiple images to capture all the details of the measured object. Once, the measurements are collected, the contrast map is identified and all the images are moved to the same coordinate $(x, y)$. In this case, the object can be located within $3 \mu \mathrm{m} \times 3 \mu \mathrm{m}$ area (or $34 \times 34$ pixels). Figure 4.9 illustrates the nine registered images translated to the same coordinate.
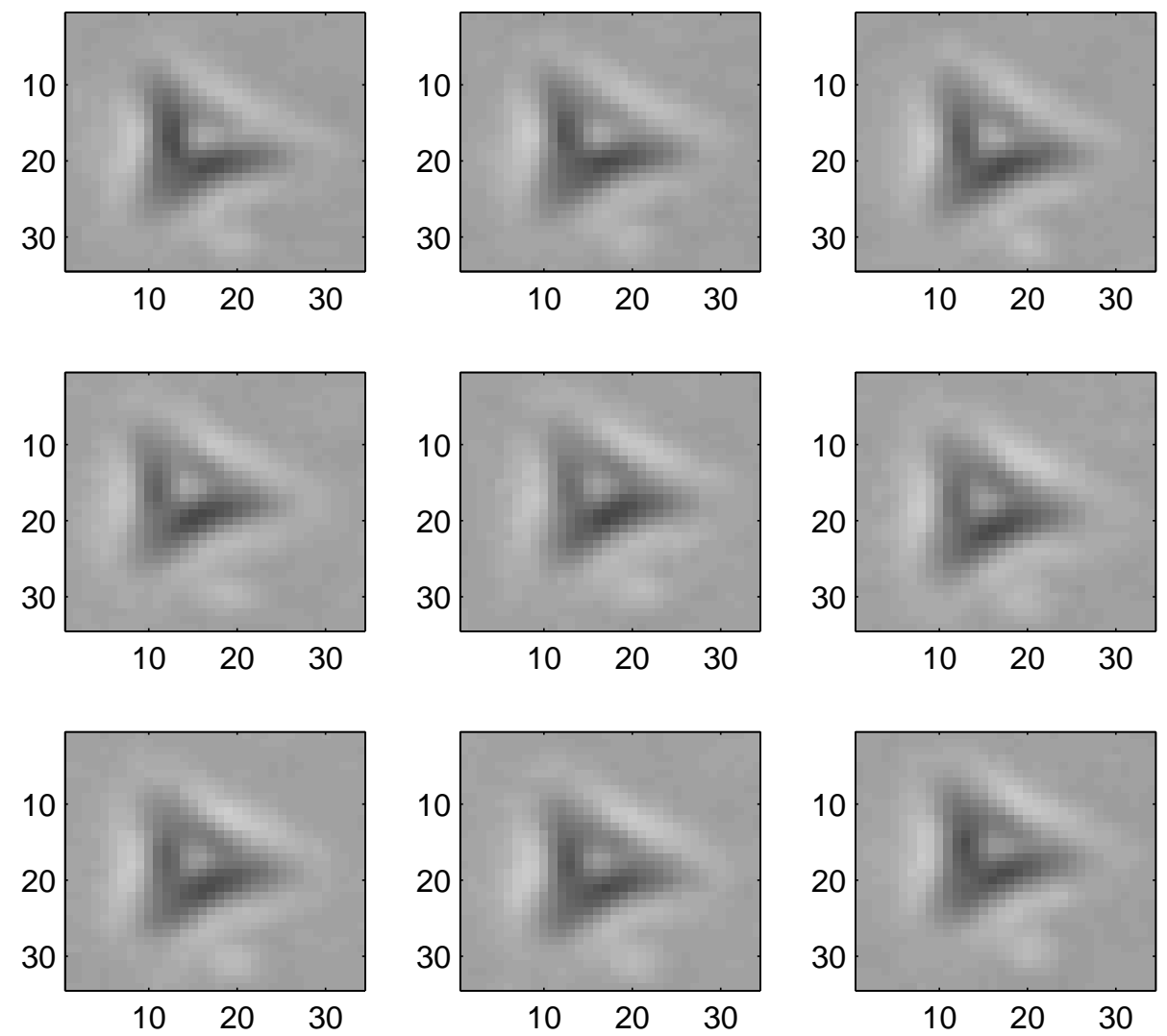

Figure 4.9 Set of nine images of a indented mark translated to the same coordinate. 

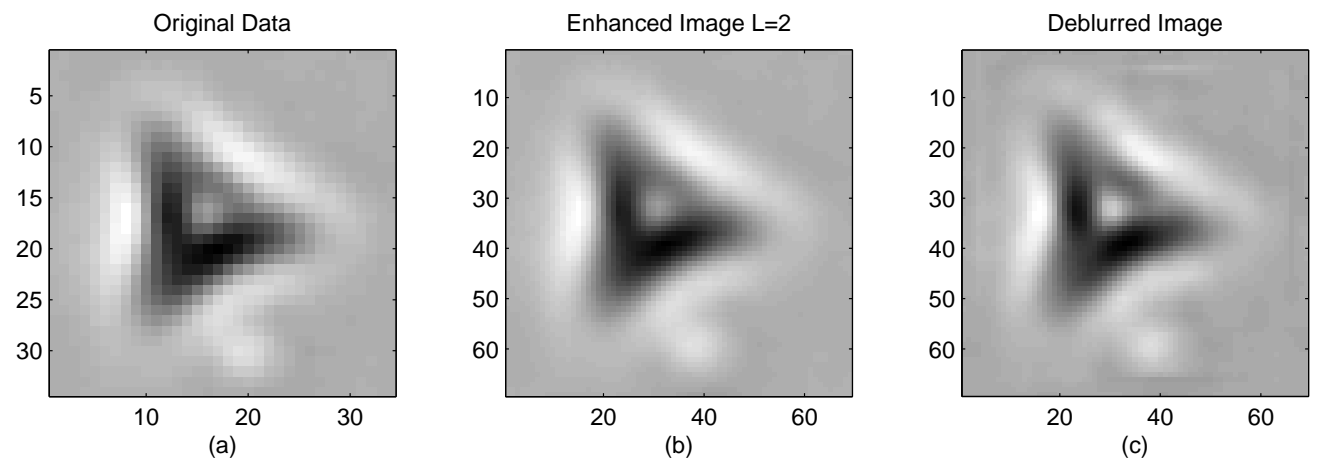

Figure 4.10 Comparison between the a) original data $34 \times 34$ with b) the enhanced image $68 \times 68$ and c) the deblurred image $68 \times 68$.

(a)

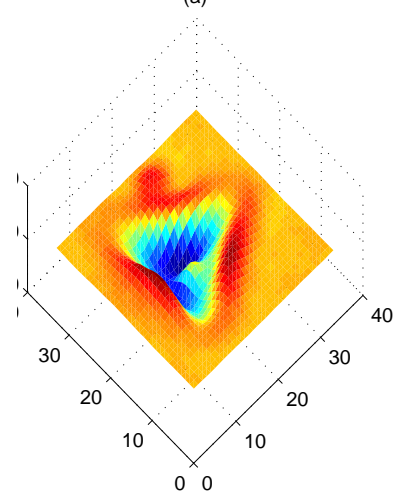

(b)

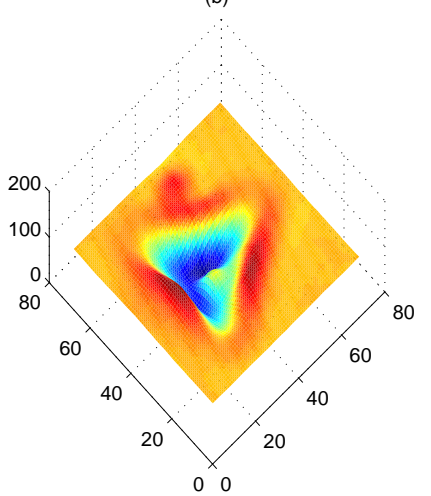

(c)

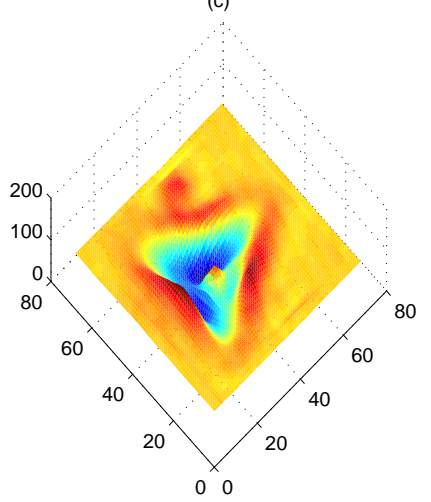

Figure 4.11 Comparison of the 3D images between the a) original data $34 \times 34$ with b) the enhanced image $68 \times 68$ and c) the deblurred image $68 \times 68$.

Once all images are located in the same coordinates, the mean of all the images is computed. The obtained low resolution image is then processed by the proposed enhance- 
ment method, the implemented enhancement ratio was $L=2$. The geometric shape of the targeted object can be further enhanced by deblurring the process data. Figure 4.10 shows comparison between the two-dimensional a) original sample, b) the processed image and c) the deblurred image. Figure 4.11 illustrates the three dimensional representation before and after processing and the deblurred 3D image.

As a conclusion, with the proposed method, the processed image shows minor imprinted structures when multiple images are processed. Also the shape and the edges after deblurring are more defined. In fact, the triangle in the middle of the triangular shape can be only recognizable after this process. 


\section{CHAPTER 5}

\section{EXPERIMENTAL RESULTS}

\subsection{Resolution Enhancement of FSR Measurement}

In order to verify the performance and effectiveness of the Proposed Resolution Enhancement Algorithm (PREA), three cases are investigated along this chapter. The first case includes two sets of height data acquired by an atomic force microscope (AFM), these two images are downsampled and reconstructed to evaluate how image quality can be improved by the proposed algorithm. By comparing the result with another algorithm, benchmark can be determined. The second set of data is a single image obtained from a Thru mode FSR array discussed in chapter 3. Objects of different geometric shapes are measured and then processed by the PREA to identify the corresponding shapes. Finally, the proposed algorithm is used to reconstruct the geometric shape of an object from multiple measurements that only include part of the complete information. The purpose of the second and third case is to prove that the resolution of a tactile sensor can be improved by the implementation of a super resolution algorithm.

\subsection{Resolution Enhancement of a Single Set of Data}

In this section, two substrates were acquired using an atomic force microscope (AFM) [37]. The material of the sample is a photosensitive polymer sheet that is used for a laser printer as the electrophotografic image belt. In this case, there is only a single image for each substrate. The sizes of the original images are $512 \times 512$ pixels in $10 \mu \mathrm{m} \times 10 \mu \mathrm{m}$. The two dimensional images are shown as Figure 5.1 and Figure 5.3. 


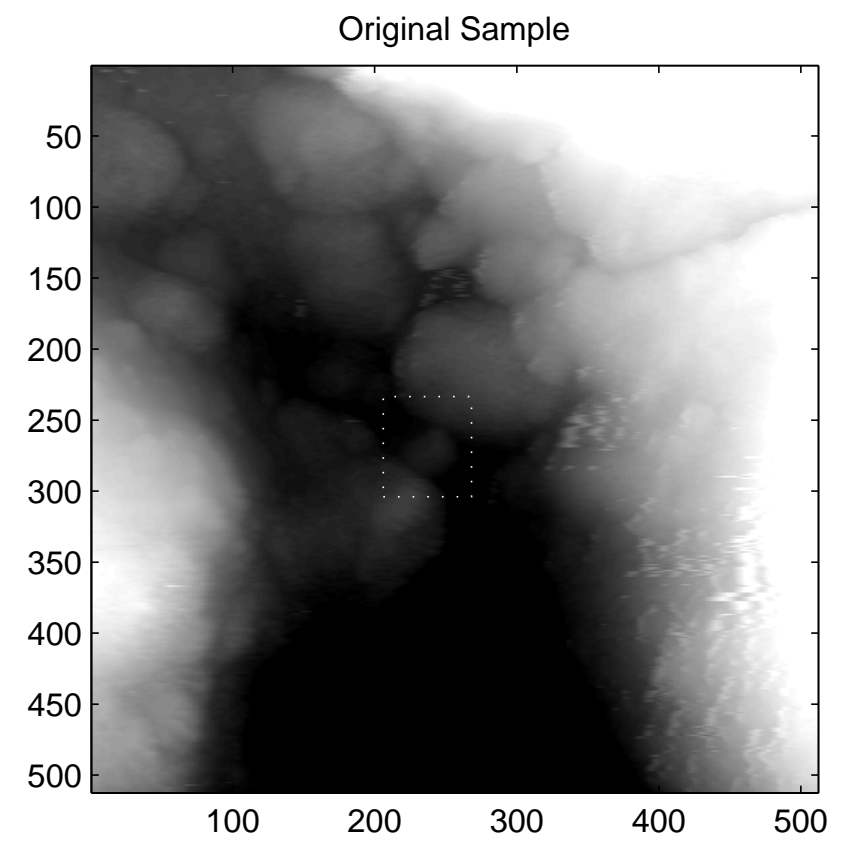

Figure 5.1 Raw data of the original $512 \times 512$ sampled output one $(10 \mu \mathrm{m} \times 10 \mu \mathrm{m})$. The dotted square shows the selected area.
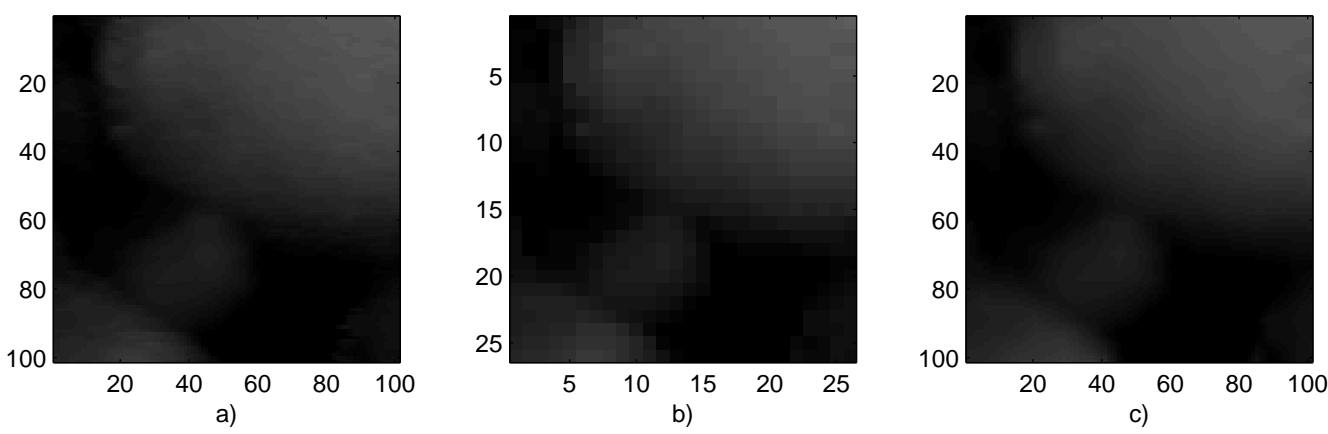

Figure 5.2 Comparison between the data of sample 1: a) Original image; b) downsampled image $25 \%$; and c) the reconstructed image. 


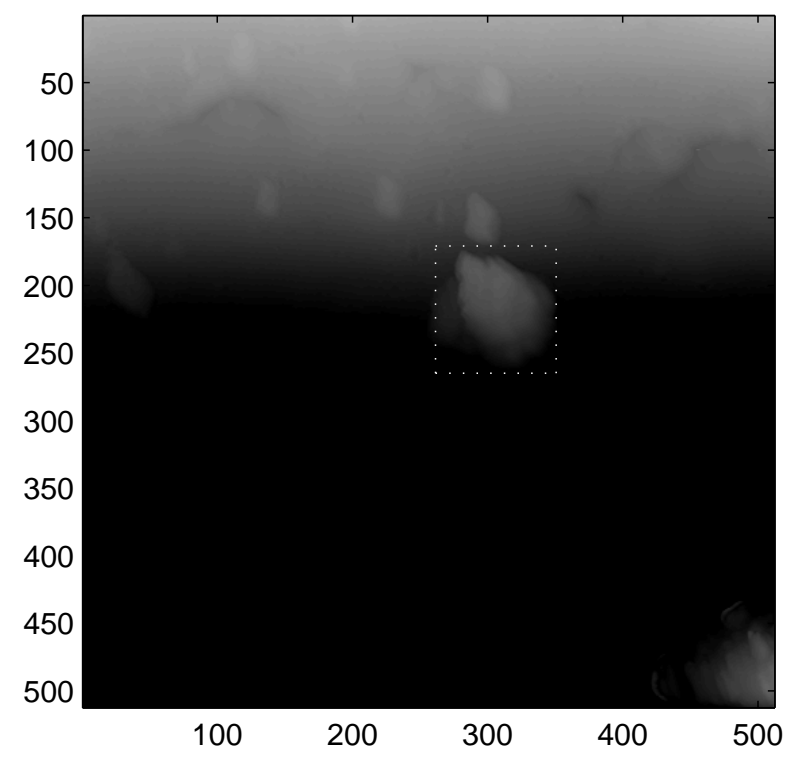

Figure 5.3 Raw data of the original 512x512 sampled output two $(10 \mu \mathrm{m} \times 10 \mu \mathrm{m})$. The dotted square shows the selected area.
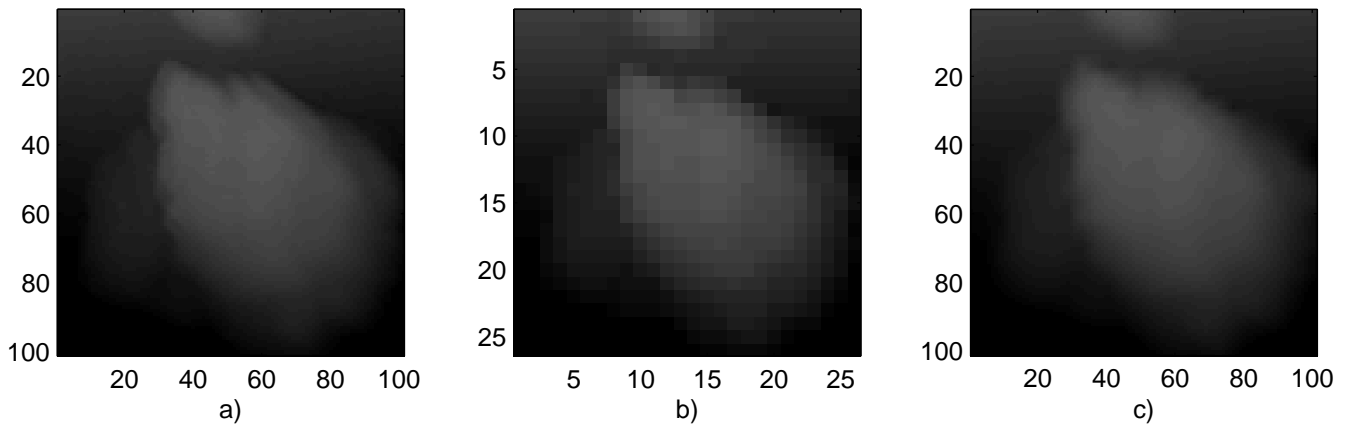

Figure 5.4 Comparison between the data of sample 2: a) Original image; b) downsampled image $25 \%$; and c) the reconstructed image. 
To verify the performance of the proposed resolution enhancement algorithm, two generic images are downsized using the method explained in the previous chapter. To evaluate the effectiveness of the algorithm, small portions of the original images with different structures are considered. The sizes of the selected areas are $100 \times 100$ pixels. Then the images are downsampled to $25 \%$ and $10 \%$ from the original samples. This implies that, the compression ratio $L$ is equal to 4 and 10. The downsampled images, which are low resolution images, are reconstructed using the proposed resolution enhancement algorithm. Figure 5.2 and Figure 5.4 illustrates the original sample, the downsized image and the reconstructed image.

\subsubsection{Parameters of Image Quality}

To determine the quality of the reconstructed images, two parameters of measuring image quality were considered: the mean squared error (MSE) and the peak signal-to-signal ratio (PSNR). Peak-signal-to-noise ratio is a parameter commonly used in image processing to compared an original signal with a reconstructed signal. Higher PSNR usually implies a better approximation of the original image. The other parameter usually used to evaluate image quality is MSE, which is

$$
M S E=\frac{1}{N^{2}} \sum_{i=1}^{N} \sum_{j=1}^{N} \mid\left(B_{(i, j)}-\left.\widehat{B}_{(i, j)}\right|^{2}\right.
$$

where $B$ is height information of the original image that contains $N \times N$ pixels and $\widehat{B}$ is the approximated height of the processed image. The peak signal-to-signal ratio (PSNR) is computed by the following equation and the units are decibels $(\mathrm{dB})$. That is

$$
P S N R=20 \cdot \log _{10}\left(\frac{P^{2}}{M S E}\right) d B
$$


where $P$ is the maximum pixel value of the image. In general, the larger PSNR means a better approximation of the processed image in relation to the original sample. To evaluate the output quality of the proposed resolution enhancement algorithm, it is suggested that the value of the PSNR is greater than $25 \mathrm{~dB}$, if the data sets are converted to grayscale images.

To verify the performance of the proposed method, PSNR between original and processed image is computed by a MATLAB script. The PSNR values are compared with the results of the adaptive sampling algorithm proposed by Cheng et al. in [37] [38] to evaluate the performance of the proposed algorithm. From the results shown in Table 5.1, the proposed method not only requires lower number of measurements but also demonstrates higher values of PSNR compared with the adaptive sampling algorithm.

\begin{tabular}{|c|c|c|c|c|}
\hline \hline & \multicolumn{2}{|c|}{ Sample 1 } & \multicolumn{2}{c|}{ Sample 2 } \\
\cline { 2 - 5 } & Comp. Ratio(\%) & PSNR(dB) & Comp. Ratio(\%) & PSNR(dB) \\
\hline \hline \multirow{3}{*}{ Proposed Algorithm } & 25 & 35.62 & 25 & 35.89 \\
\cline { 2 - 5 } & 10 & 27.17 & 10 & 26.84 \\
\hline \multirow{3}{*}{ Adaptive Sampling } & 43 & 27.37 & 87 & 34.08 \\
\cline { 2 - 6 } & 25 & 25.72 & 12 & 28.2 \\
\hline \hline
\end{tabular}

Table 5.1 Performance comparison between the proposed enhancement resolution algorithm with the adaptive sampling algorithm. 


\subsection{Resolution Enhancement of a Sensor Array}

The Proposed Resolution Enhancement Algorithm (PREA) has demonstrated excellent results in the previous section that can be used to accurately enhance the resolution of sampled data. Thus, the PREA is suitable to increase the resolution of images acquired by tactile sensor arrays, which typically have limited resolution.

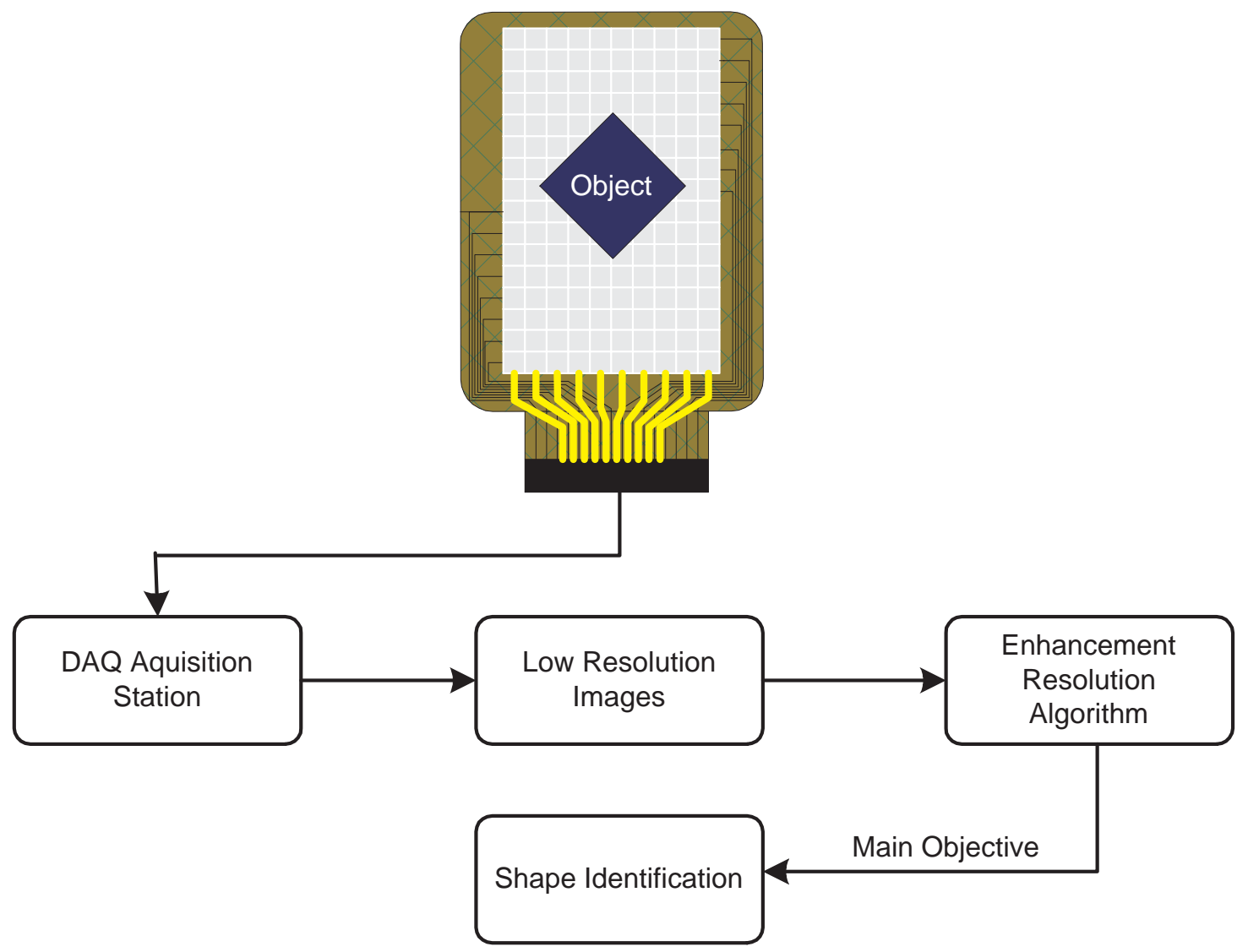

Figure 5.5 Procedure to improve the resolution of the input data provided by a Thru mode FSR array.

The adopted tactile sensor array is a Thru mode FSR array provided by [32]. This array is composed of 160 sensors that is $16 \times 10$ matrix. Due to the low resolution and re- 
peatibility of tactile devices, a resolution enhancement algorithm is required. The procedure of data collection and data processing is shown as Figure 5.5. While measuring, the object is placed on the surface of the Thru mode FSR array. The measurements of individual sensors are then collected by a DAQ station. However, due to the low resolution of the sensor array, the geometric shape of the object can barely be identified even after all the reading are taken. With PREA, the resolution of the image can be greatly improved. Thus, the geometric dimension and force distribution can be identified.

\subsubsection{Processing of a Single Set of Data}

To verify that the PREA can be used in all kind of scenarios, five objects with different shapes and sizes are used in this section. In the experiments, the object is placed on the surface of a Thru mode FSR array with a $86 \mathrm{~N}$ force. The force was calibrated by a force gauge that is mentioned in Chapter 3. The individual readings of all sensors are then taken by the DAQ station. Afterwards, the raw image of every object is saved. The resolution of the image is $16 \times 10$ pixels, which is poor. The image is then processed by the PREA. To further improve the image quality, multiple images will be used for missing parts in a single acquired image.

The first object used is an annulus shown in Figure 5.6. The processed results are shown in Figure 5.7. It is clear that the geometric shape of the annulus can be outlined with a single measurement. The image can be further enhanced with a deblurring algorithm. The three dimensional images of the annulus are illustrated in Figure 5.8. This result can also be further clarified with multiple measurements. 


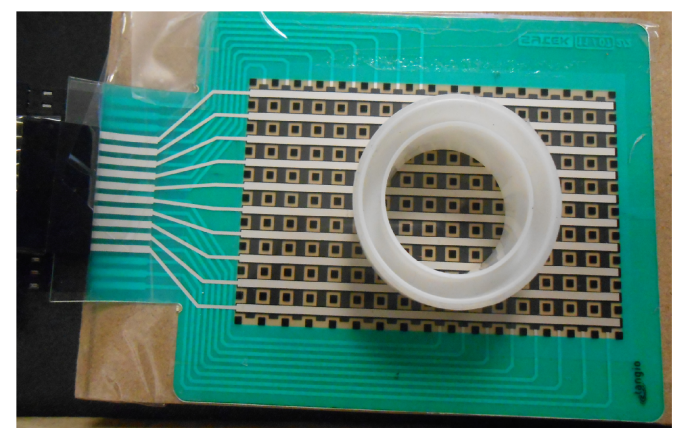

(a)

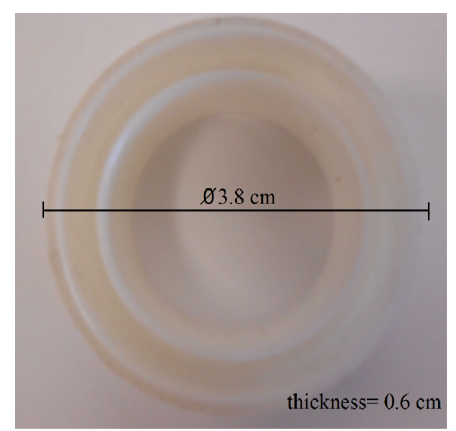

(b)

Figure 5.6 Annulus: a) Object on the top of the Thru mode FSR array and b) dimensions of the annulus.

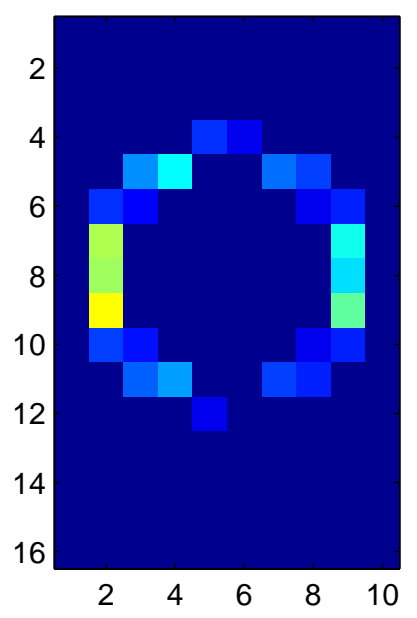

(a)

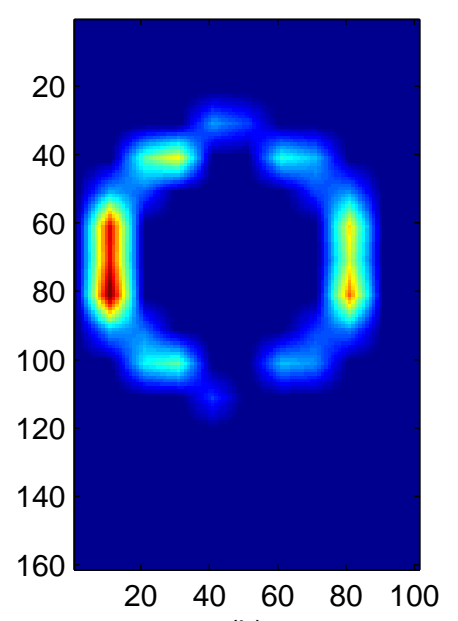

(b)

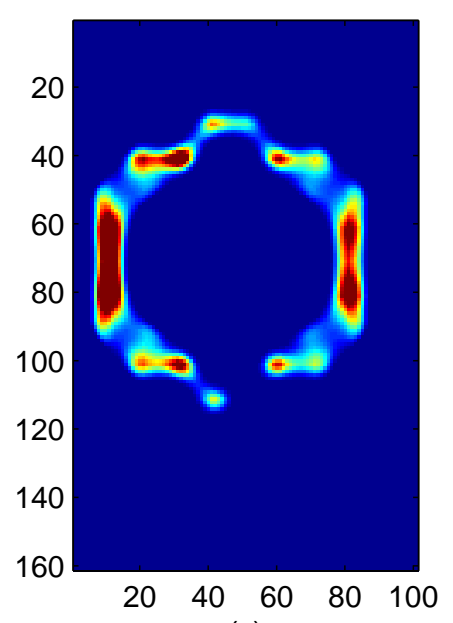

(c)

Figure 5.7 Results produced from a single image of an annulus: a) original sample; b) processed data with PREA where $L=10$; and c) the deblurred image. 
(a) Processed Data

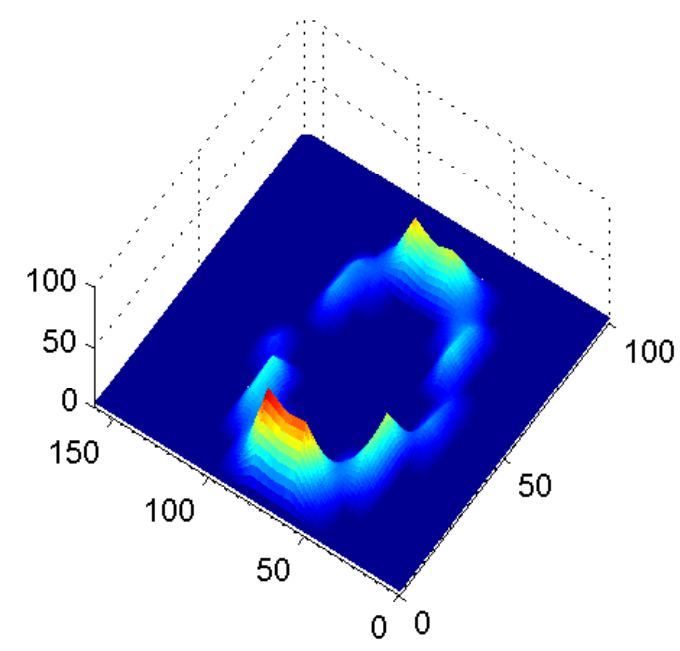

(b) Deblurred Image

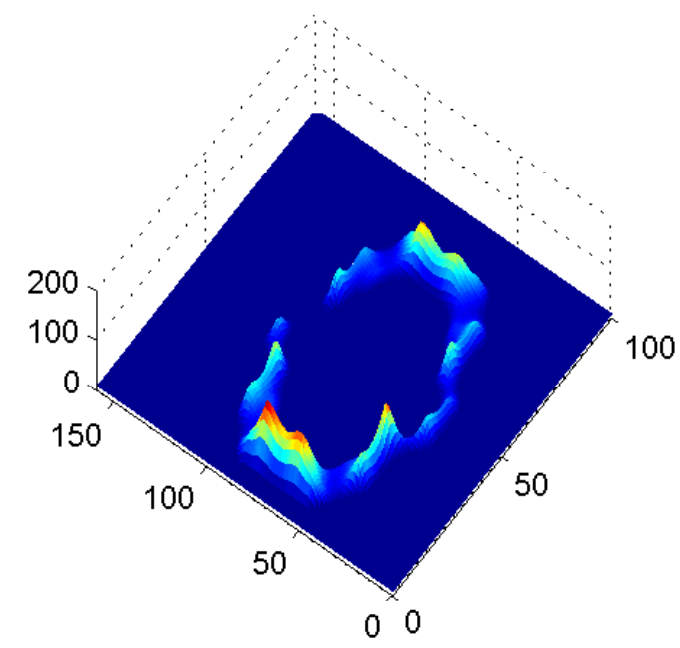

Figure 5.8 Three dimensional images of the annulus of a) the processed data and b) the deblurred image.

The second object is a letter "L". Figure 5.9 shows the shape and dimensions of this object. The results of the enhanced and 3D images are shown in Figure 5.10 and Figure 5.11.

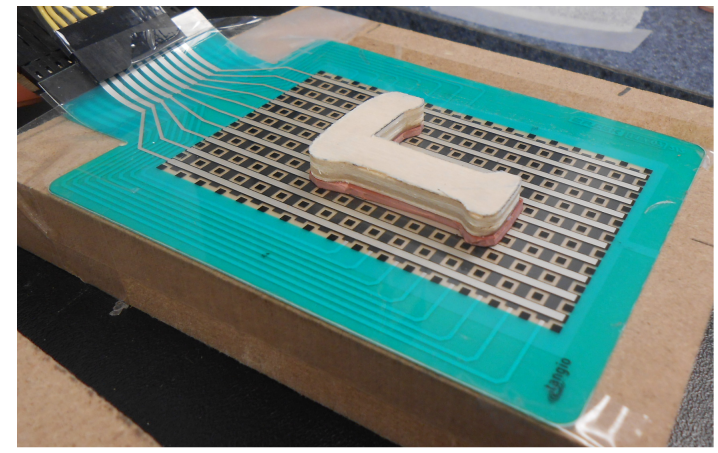

(a)

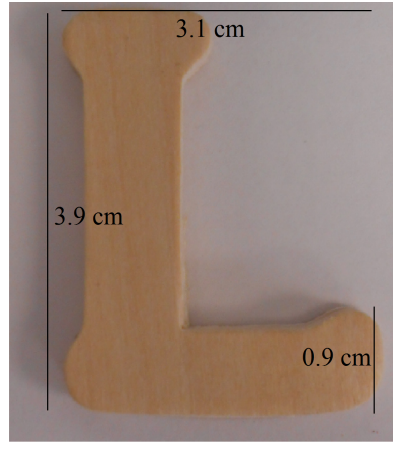

(b)

Figure 5.9 Letter "L": a) Object on the top of the Thru mode FSR array and b) dimensions of the letter "L". 


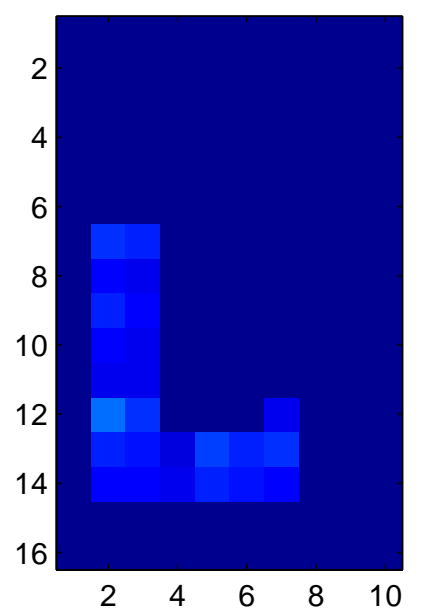

(a)

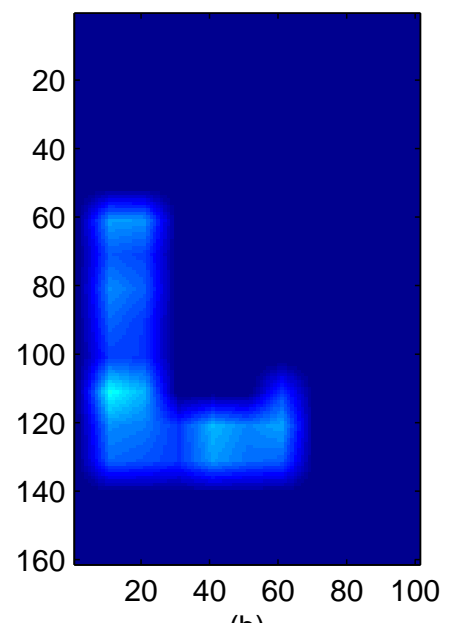

(b)

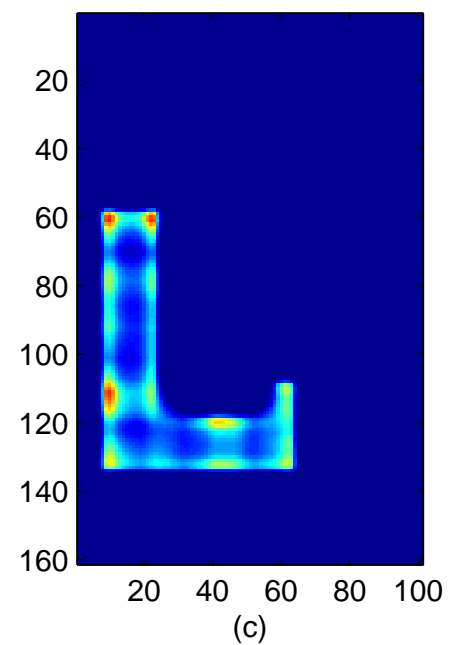

(c)

Figure 5.10 Results produced from a single image of the letter "L": a) original sample; b) processed data with PREA where $L=10$; and c) the deblurred image.

(a) Processed Data

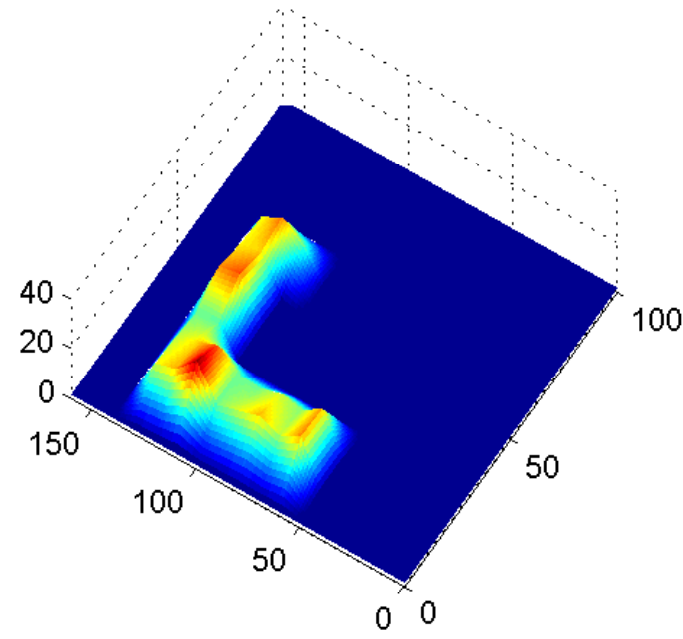

(b) Deblurred Image

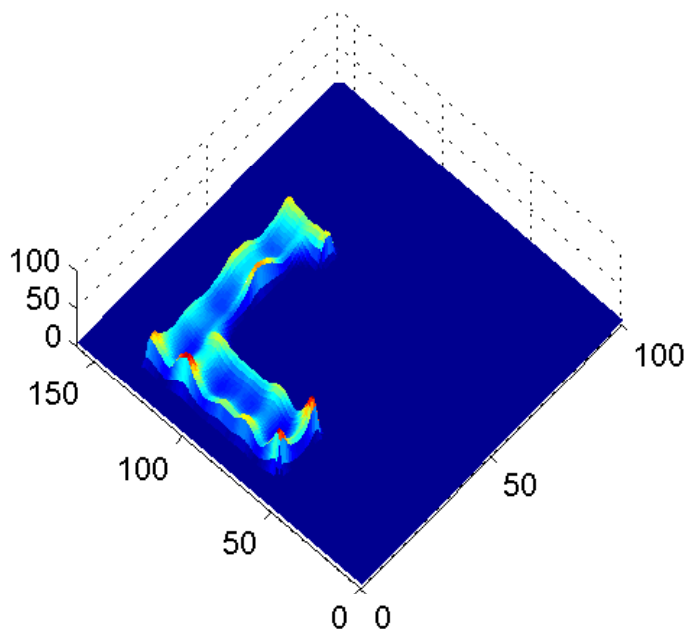

Figure 5.11 Three dimensional images of the letter "L" of a) the processed data and b) the deblurred image. 
The third object is a letter "V". Figure 5.12 shows the shape and dimensions of this object. The results of the processed and 3D images are shown in Figure 5.13 and Figure 5.14.

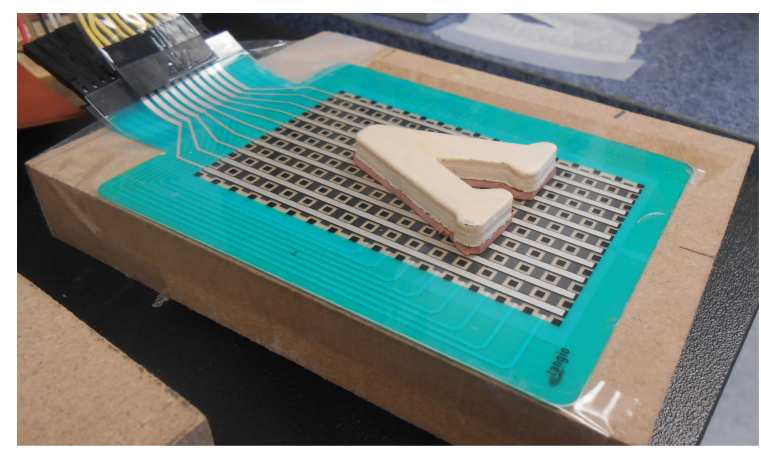

(a)

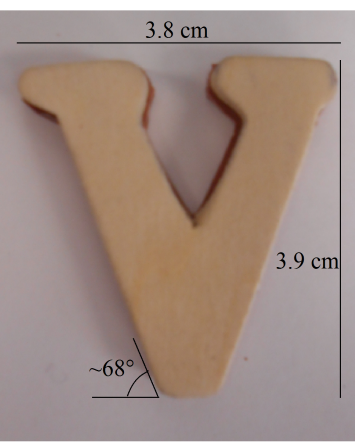

(b)

Figure 5.12 Letter "V": a) Object on the top of the Thru mode FSR array and b) dimensions of the letter "V".

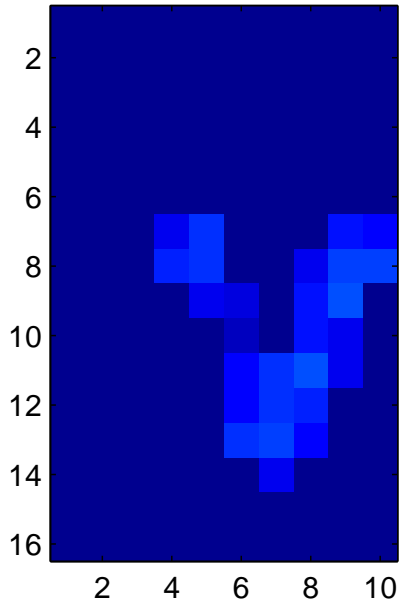

(a)

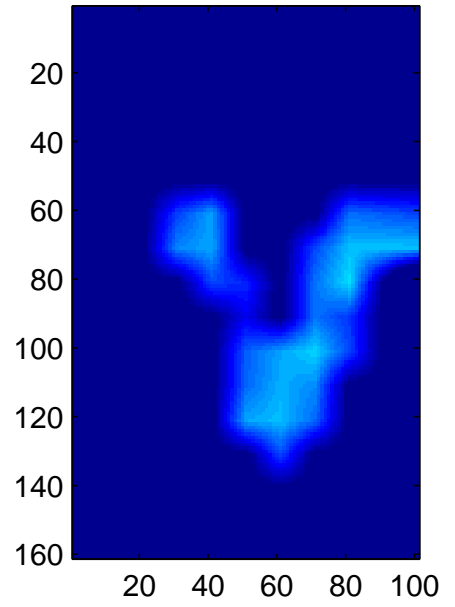

(b)

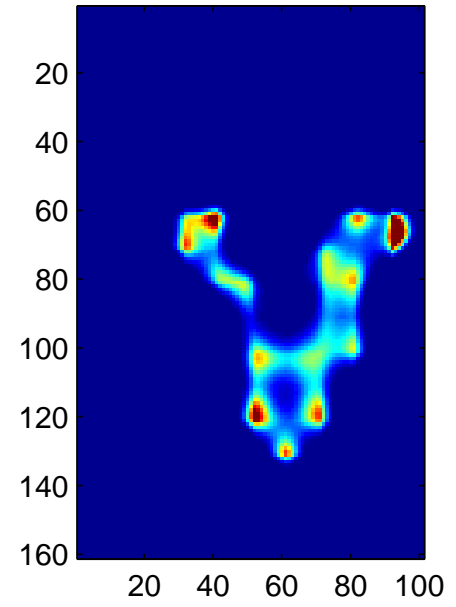

(c)

Figure 5.13 Results produced from a single image using the Letter "V": a) original sample; b) processed data with PREA where $L=10$; and c) the deblurred image. 
(a) Processed Data

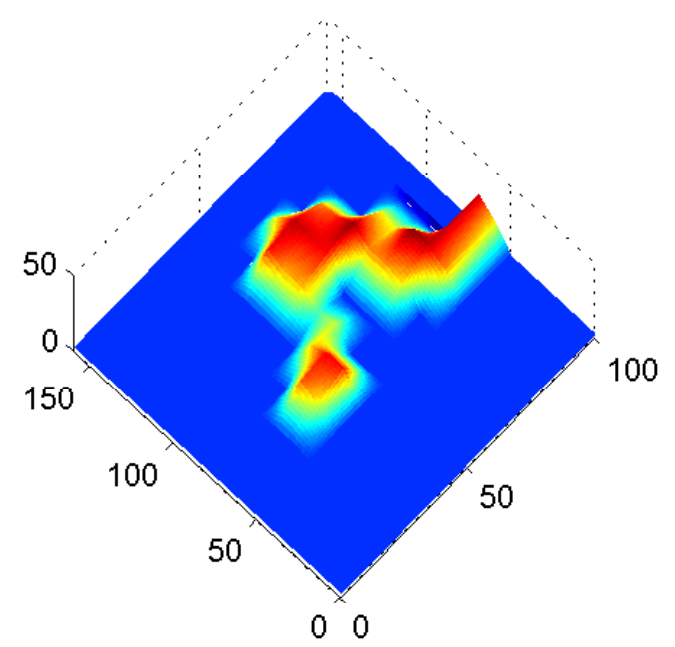

(b) Deblurred Image

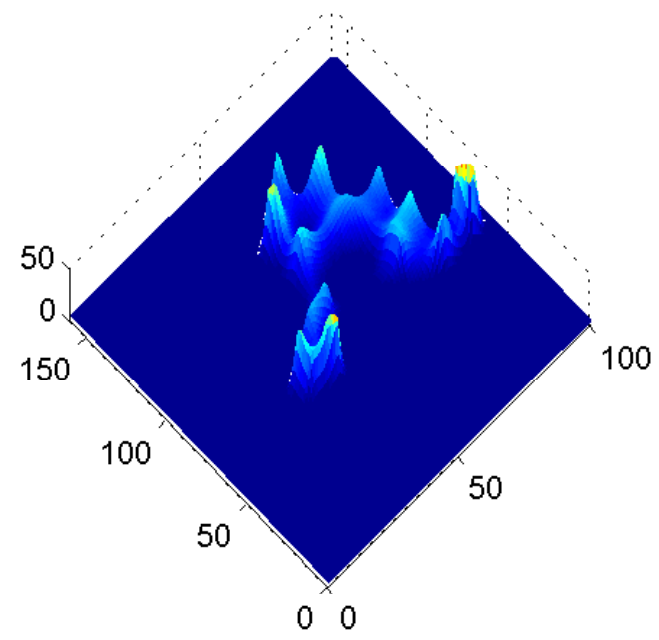

Figure 5.14 Three dimensional images of the letter "V" of a) the processed data and b) the deblurred image.

The fourth object is a letter "T". Figure 5.15 shows the shape and dimensions of this object. The results of the processed and 3D images are shown in Figure 5.16 and Figure 5.17.

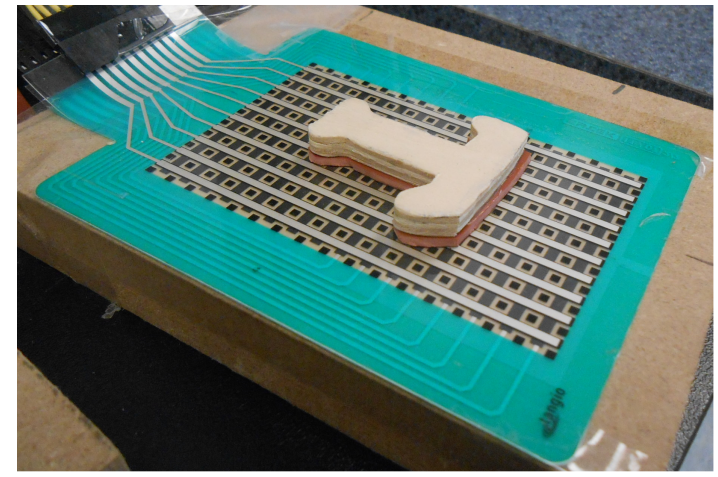

(a)

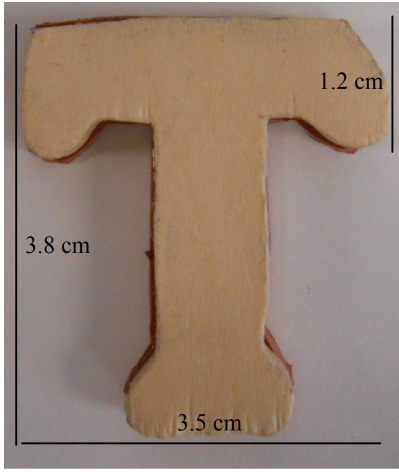

(b)

Figure 5.15 Letter "T": a) Object on the top of the Thru mode FSR array and b) dimensions of the letter " $\mathrm{T}$ ". 


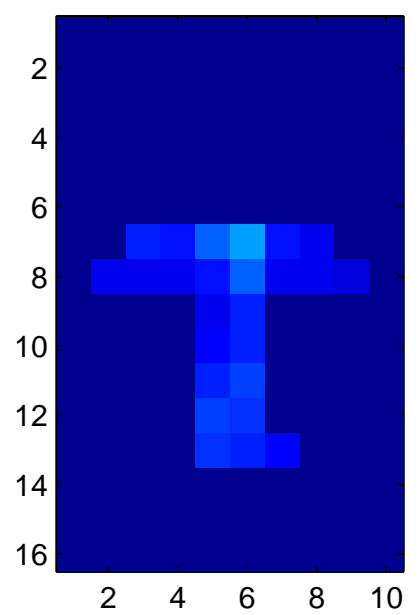

(a)

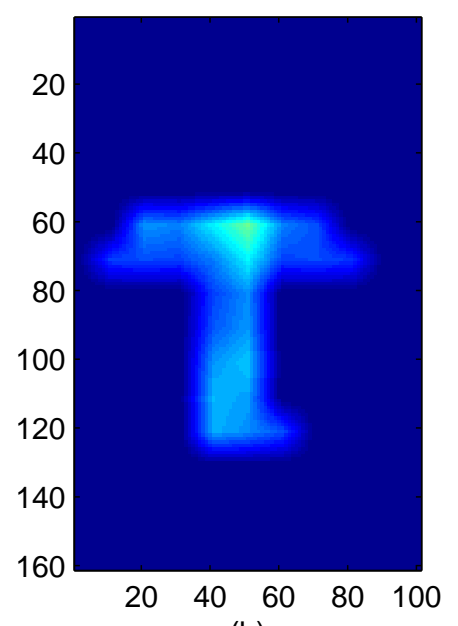

(b)

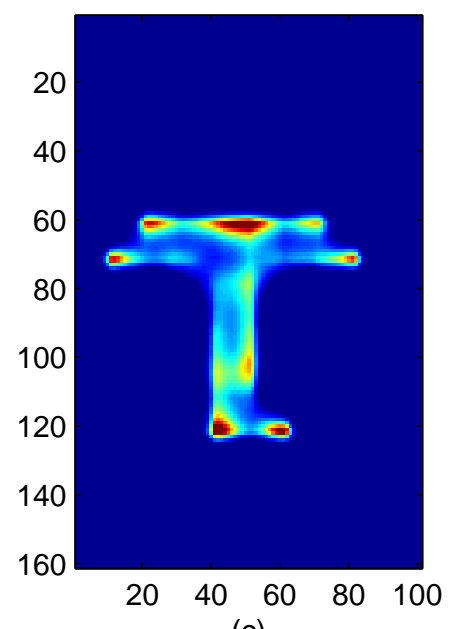

(c)

Figure 5.16 Results produced from a single image using the Letter "T": a) original sample; b) processed data with PREA where $L=10$ and c) the deblurred image.

(a) Processed Data

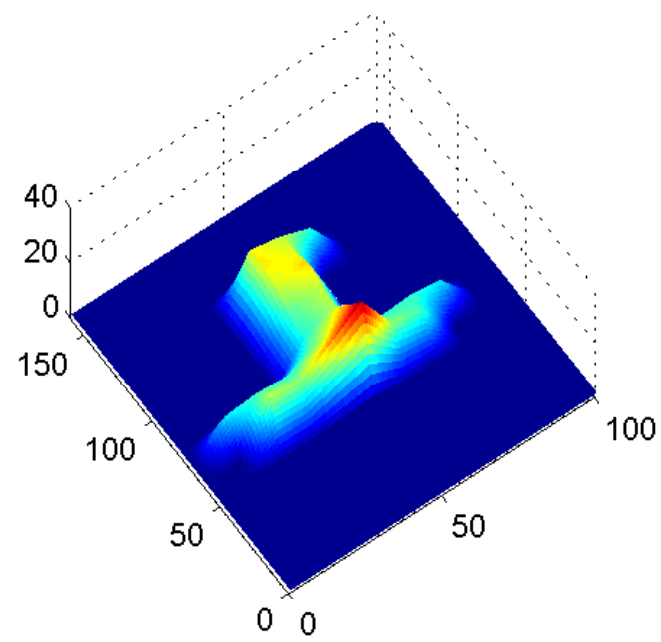

(b) Deblurred Image

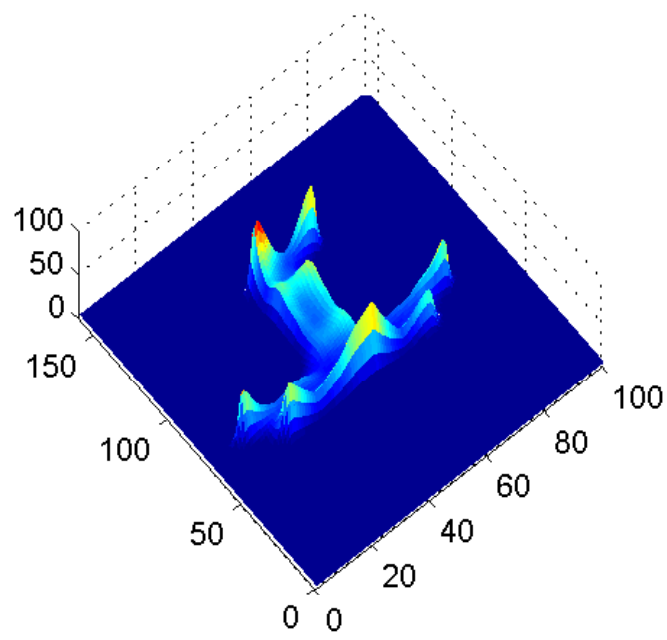

Figure 5.17 Three dimensional images of the letter "T" of a) the processed data and b) the deblurred image. 
Finally, the last object is a letter "C". Figure 5.18 shows the shape and dimensions of this object. The results of the processed image and 3D diagrams are shown in Figure 5.19 and Figure 5.20.

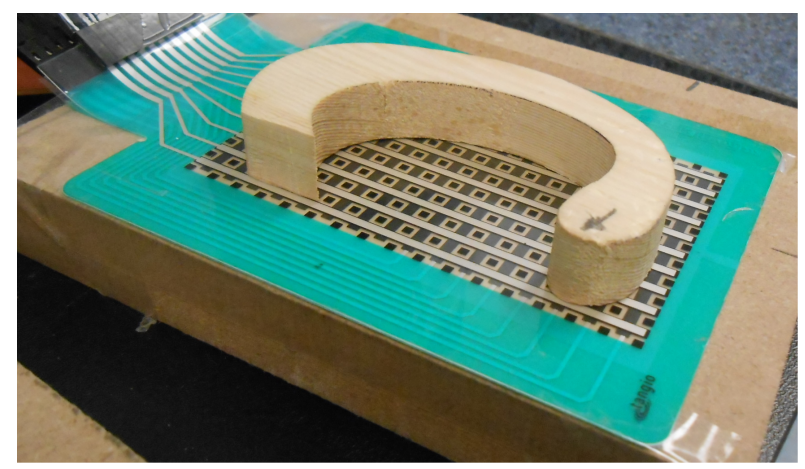

(a)

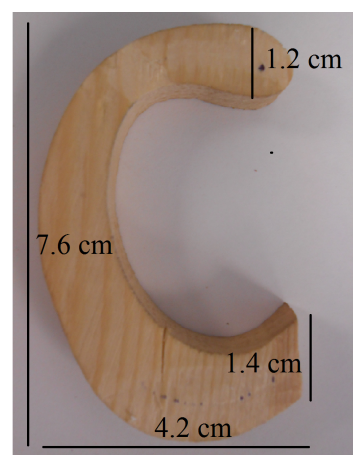

(b)

Figure 5.18 Letter "C": a) Object on the top of the Thru Mode FSR array and b) dimensions of the letter $\mathrm{C}$.
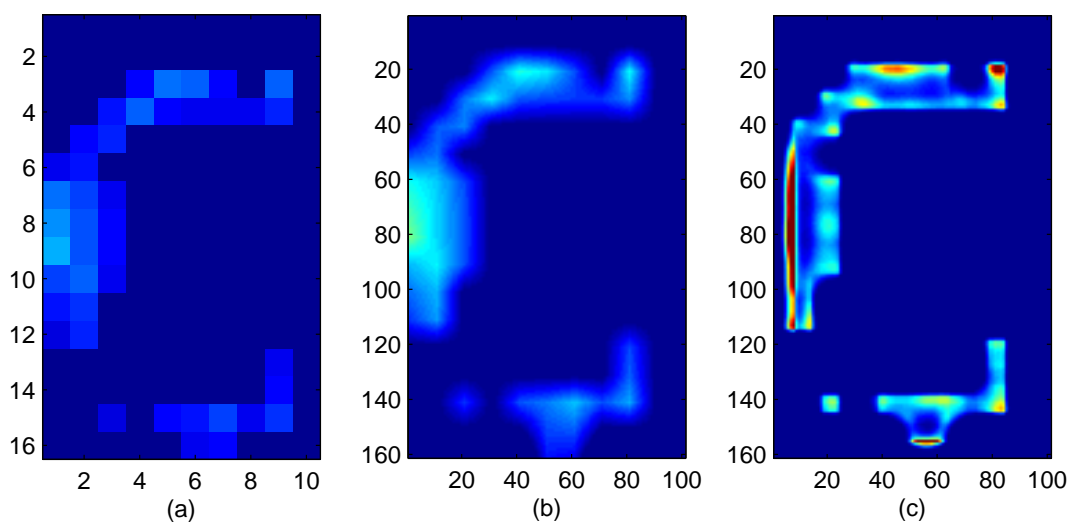

Figure 5.19 Results produced from a single image using the Letter "C": a) original sample; b) processed data with PREA where $L=10$; and c) the deblurred image. 
(a) Processed Image

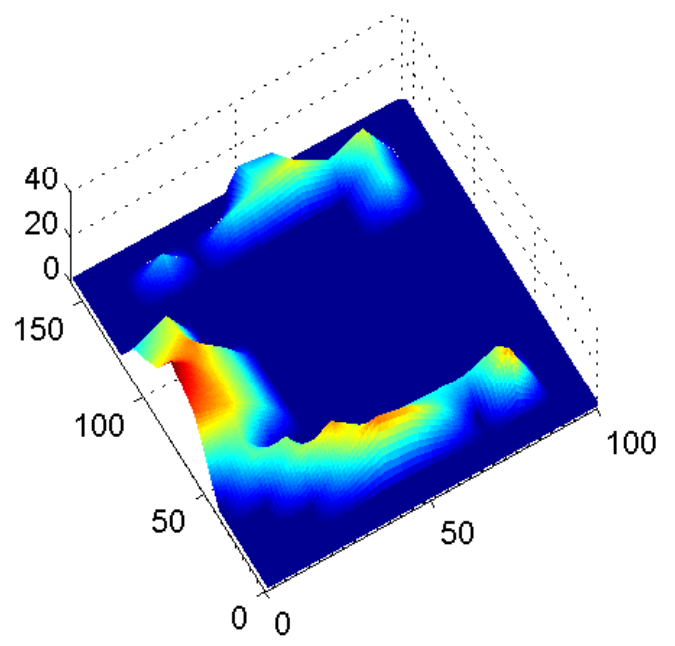

(b) Deblurred Image

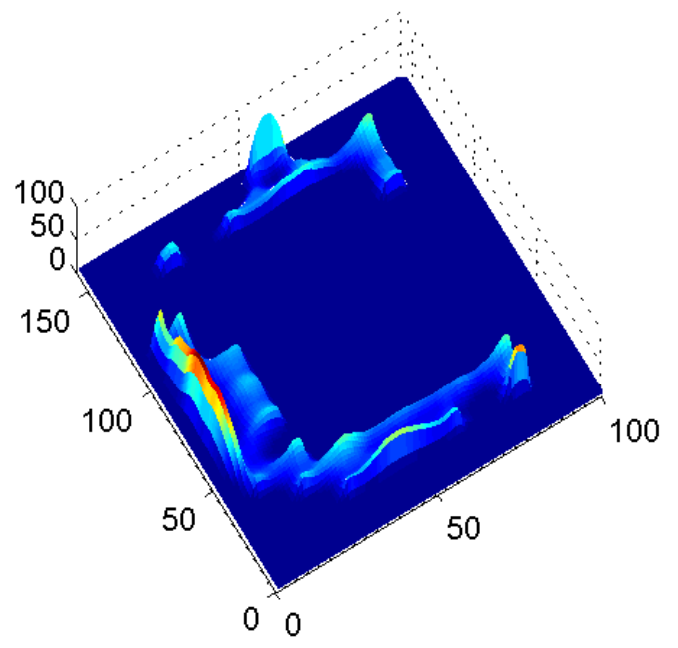

Figure 5.20 Three dimensional images of the letter "C" of a) the processed data and b) the deblurred image.

\subsubsection{Processing of Objects with Multiple Sets of Data}

The results after processing and deblurring a single image is usually good enough to identify the shape of the targeted object. However, a single image can sometimes miss information due to two important reasons: the low repeatibility of the tactile sensor and the physical malformations of the analyzed objects. For this reason, more images need to be acquired to collect all the required information missing in other images. After multiple measurements of the same object are processed, it is anticipated to generate a more reliable and accurate result.

In each case, 12 measurements were collected from every object. However, the object can be translated to different positions on the surface of the Thru Mode FSR array while the data is collected by the DAQ station. Thus, once the measurements are taken, all the images need to be shifted to the common reference. The measurements are then averaged to generate the results that contains all the information processed by the PREA. The detail procedure can be found in Chapter 4. To ensure the improvement, multiple images collected 
from annulus are shown in Figure 5.21. After translation and averaging, the processed and deblurred images are shown in Figure 5.22. The 3D images are demonstrated in Figure 5.23 as well. It is clear the results of averaging all the measurements actually compensate for all missing pixels, which greatly enhance the details of sampled object. In this particular case, the object was shifted using small increments, approximately one pixel along the $x$ and/or $y$ axis.
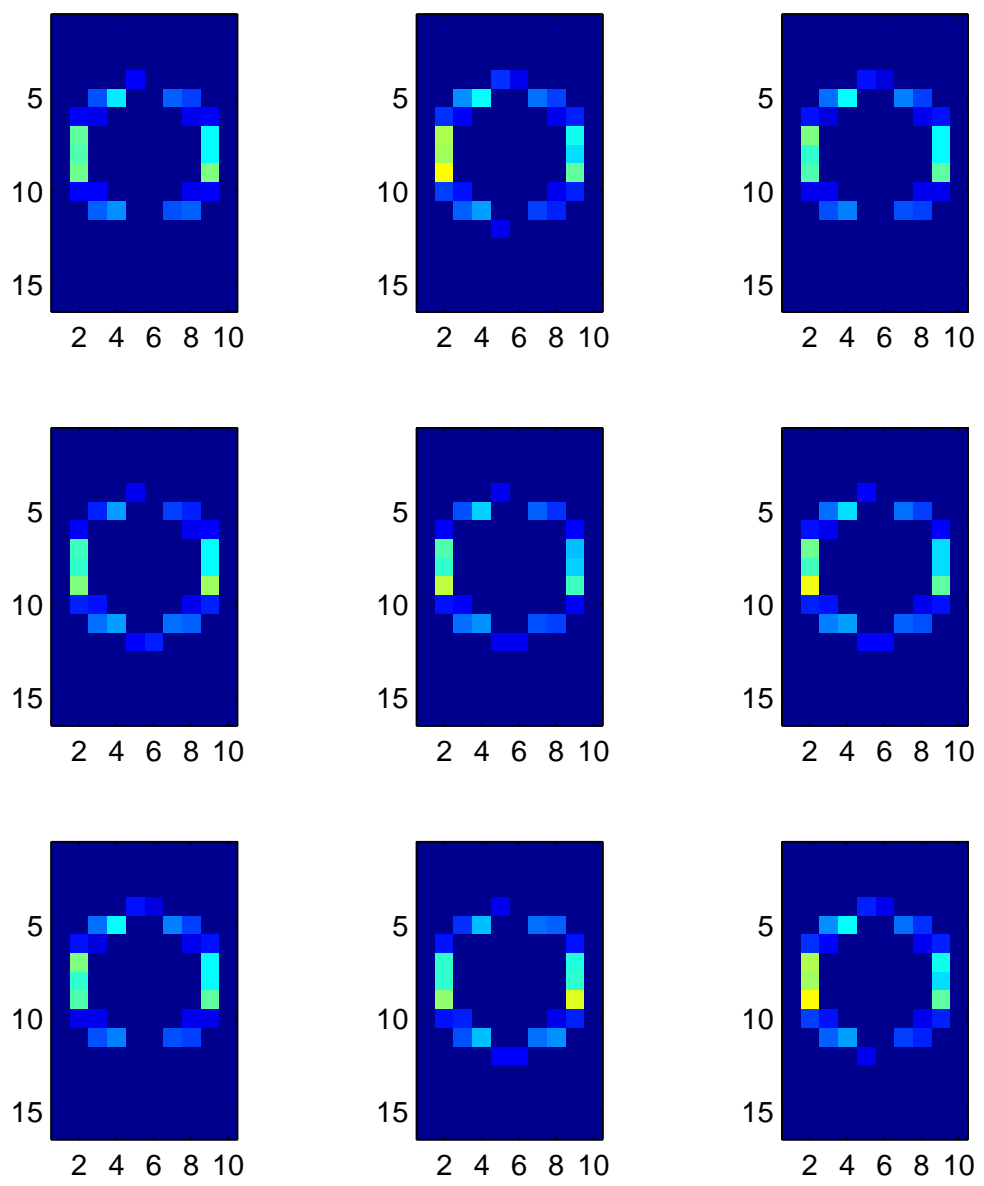

Figure 5.21 Images of nine measurements of an annulus using a Thru mode FSR array. 


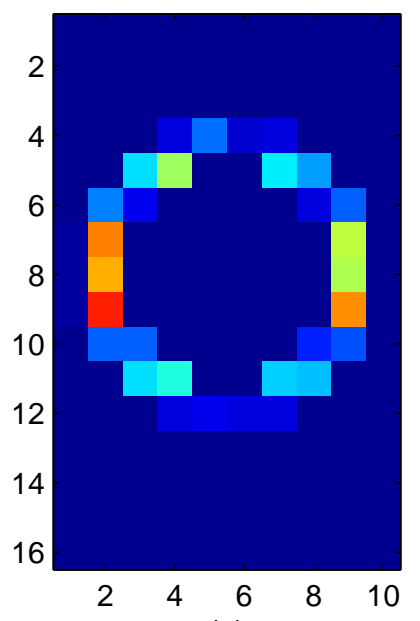

(a)

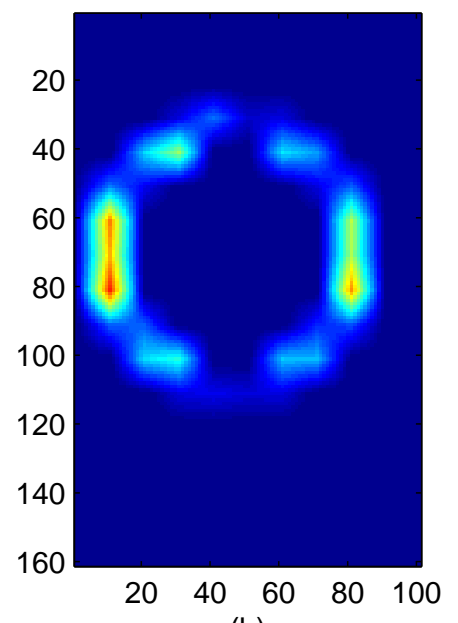

(b)

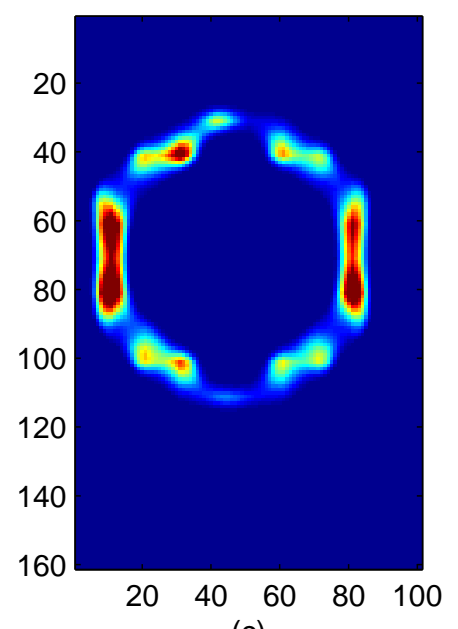

(c)

Figure 5.22 Results produced from multiple images of an annulus: a) averaged image; b) processed data with PREA where $L=10$; and c) the deblurred image.

(a) Processed Data

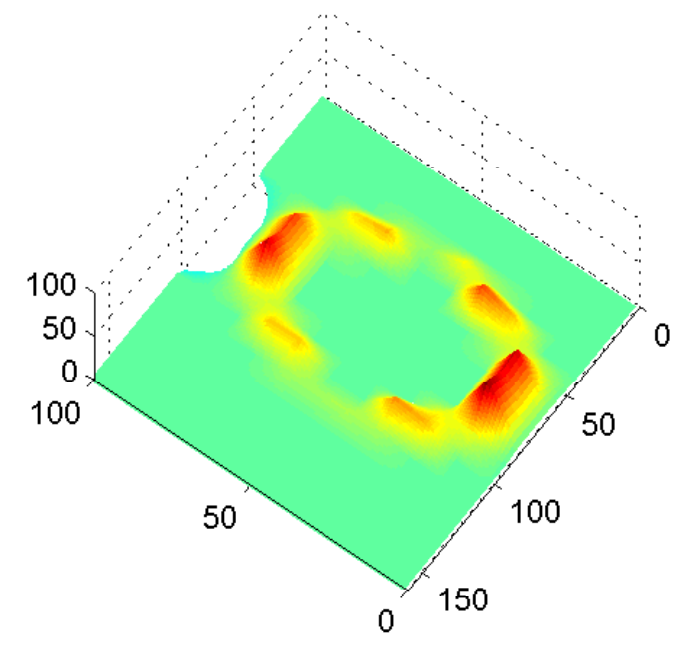

(b) Deblurred Image

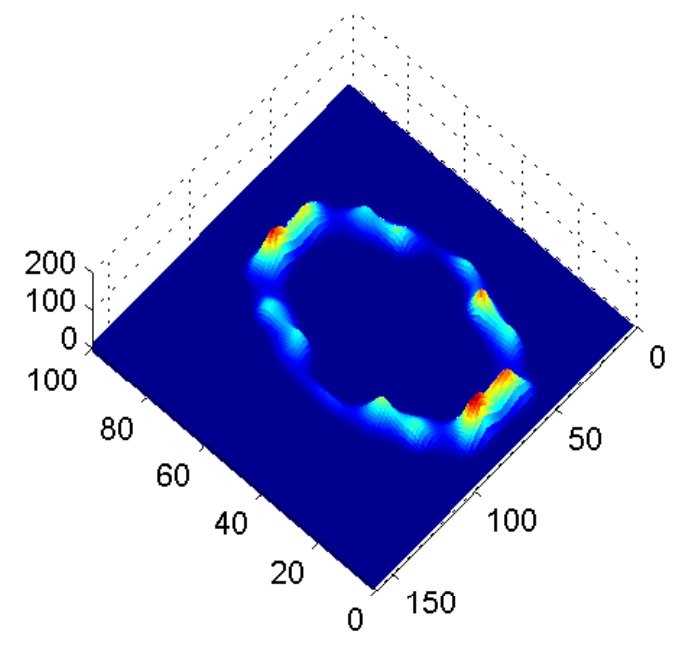

Figure 5.23 Three dimensional images of an annulus of a) processed data and b) the deblurred image. 
To verify the performance of the proposed method, multiple object have been used in this section. Figure 5.24, Figure 5.25 and Figure 5.26 illustrate the raw measurement, processed data and 3D images of letter "L" with $86 \mathrm{~N}$ applied. In order to show that the PREA is robust and efficient enough, the traslation was done with larger increments along $x$ and/or $y$ axis.
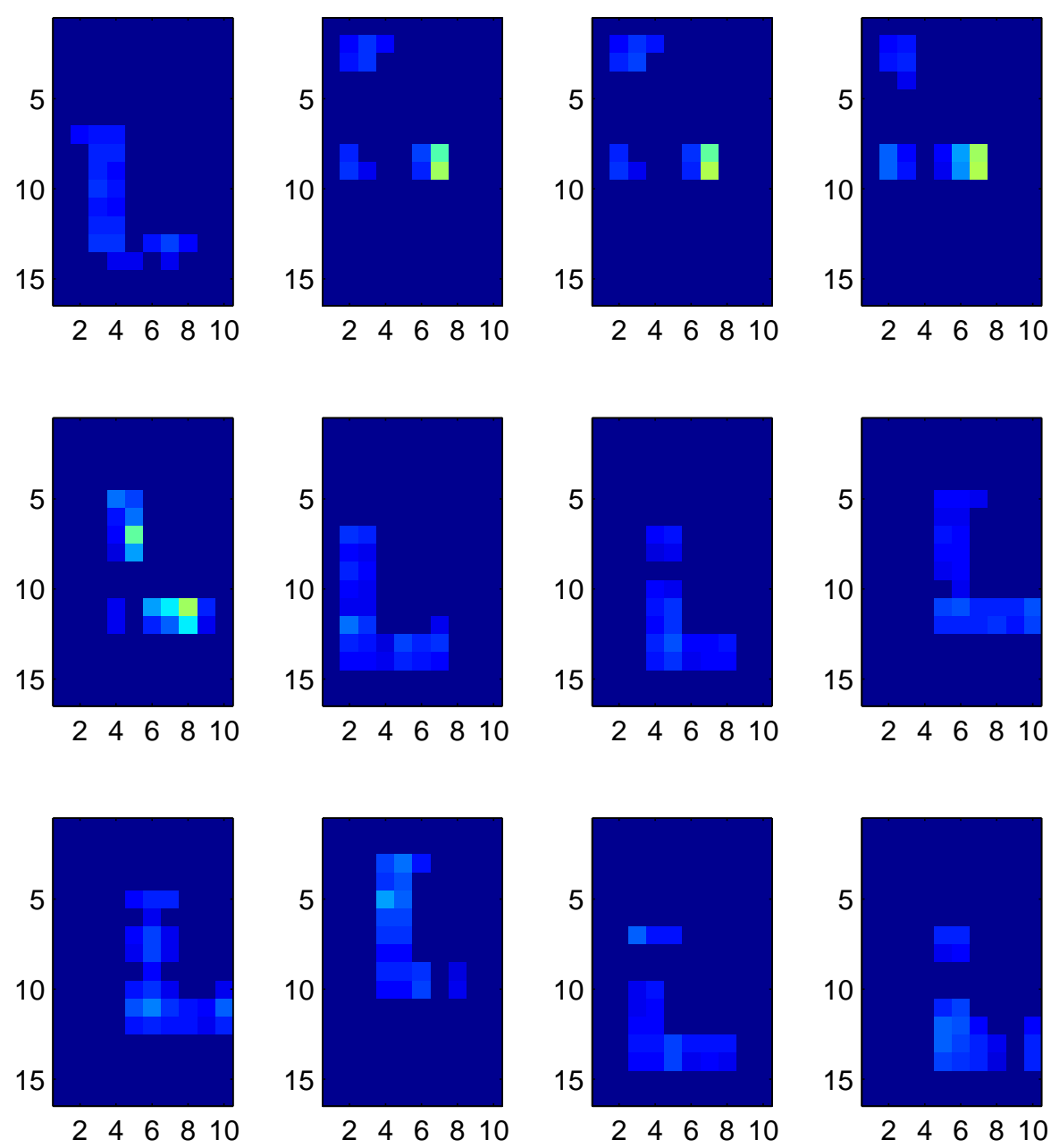

Figure 5.24 Set of 12 measurements of the letter "L" using a Thru mode FSR array. 


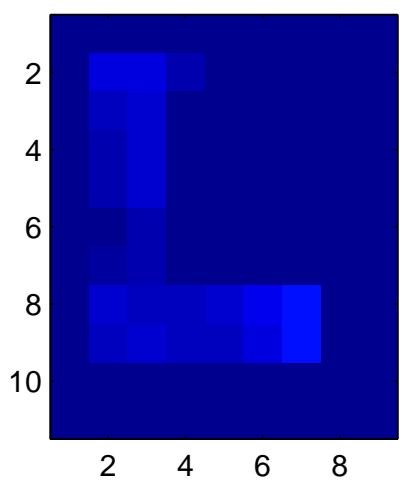

(a)

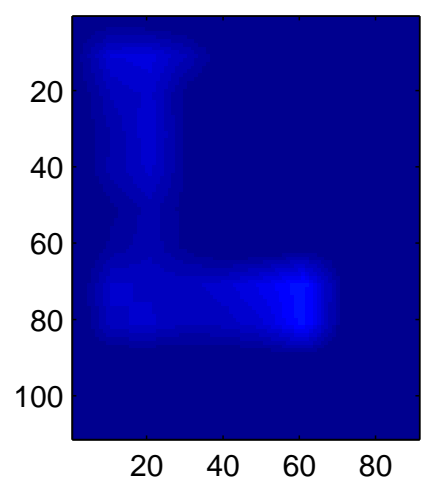

(b)

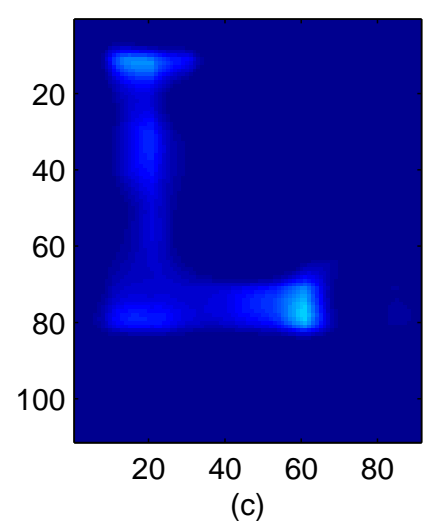

(c)

Figure 5.25 Results produced from multiple images of the letter "L": a) averaged image; b) processed data with PREA where $L=10$; and c) the deblurred image.

(a) Processed Image

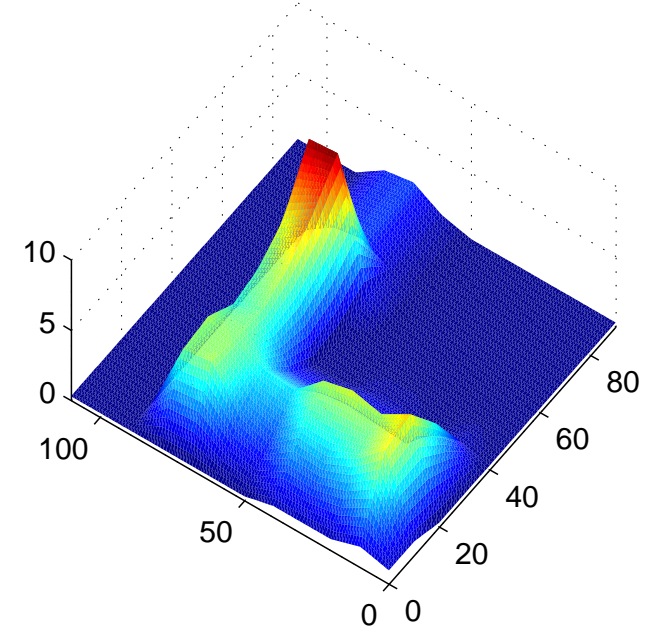

(b) Deblurred Image

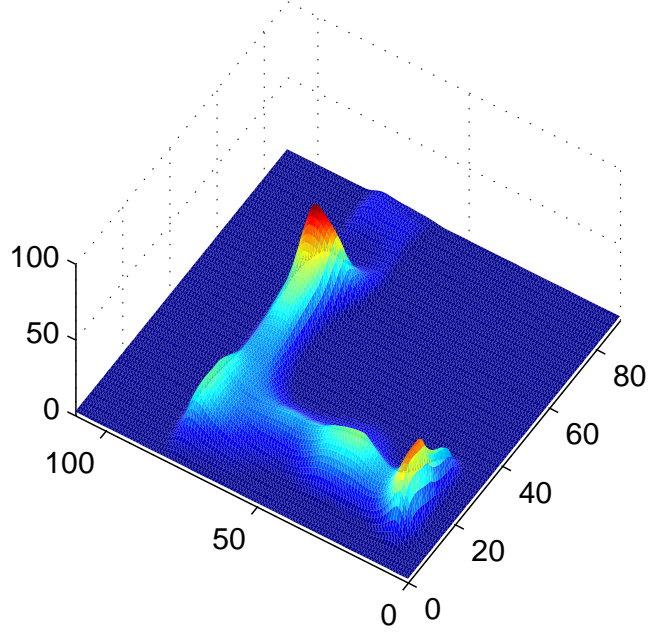

Figure 5.26 Three dimensional images of the letter "L" of a) processed data and b) deblurred image. 
Figure 5.27, Figure 5.28 and Figure 5.29 demonstrate another example of raw data, processed measurements and 3D images of letter "V".
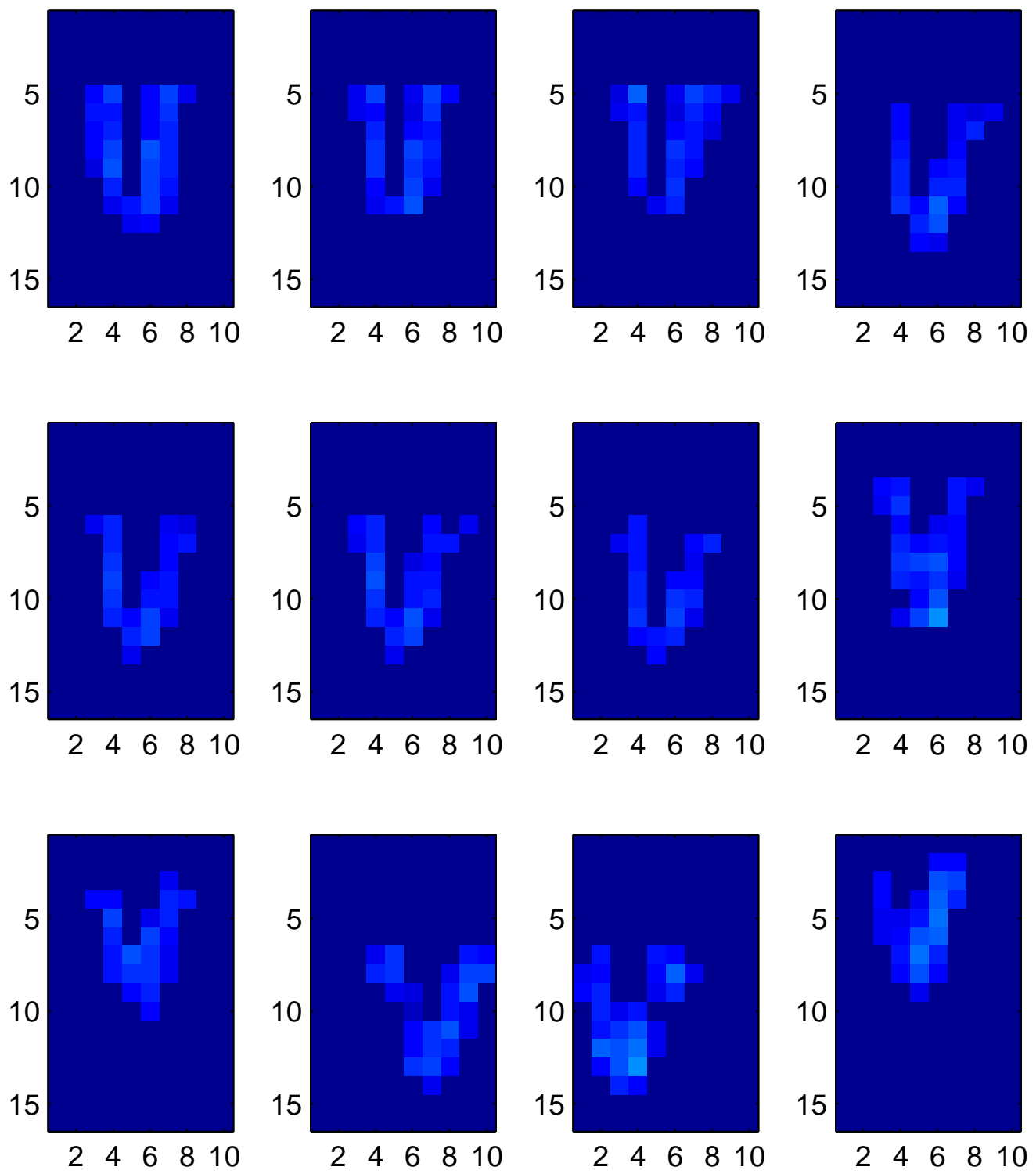

Figure 5.27 Set of 12 measurements of the letter "V" using a Thru mode FSR array. 


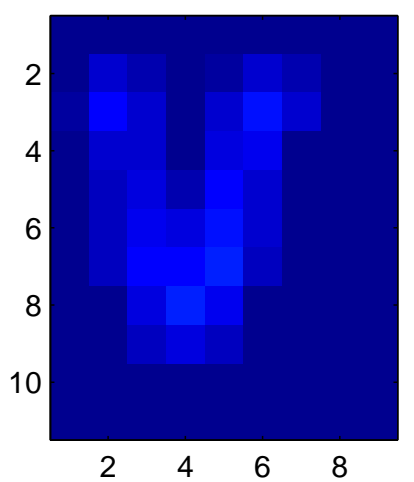

(a)

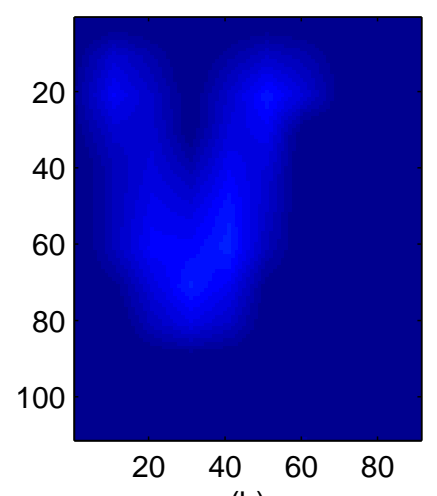

(b)

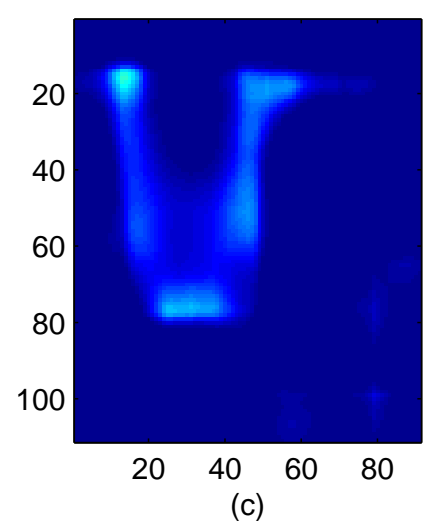

(c)

Figure 5.28 Results produced from multiple images of the letter "V". a) averaged image; b) processed data with PREA where $L=10$; and c) the deblurred image.

(a) Processed Image

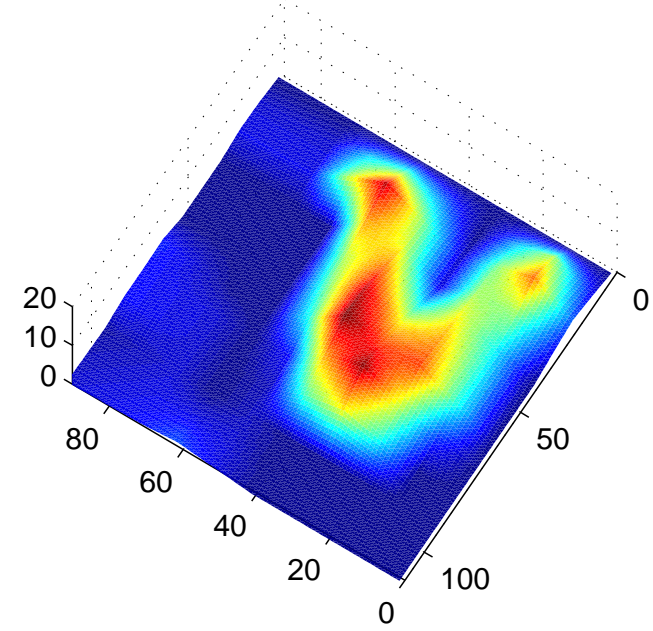

(b) Deblurred Image

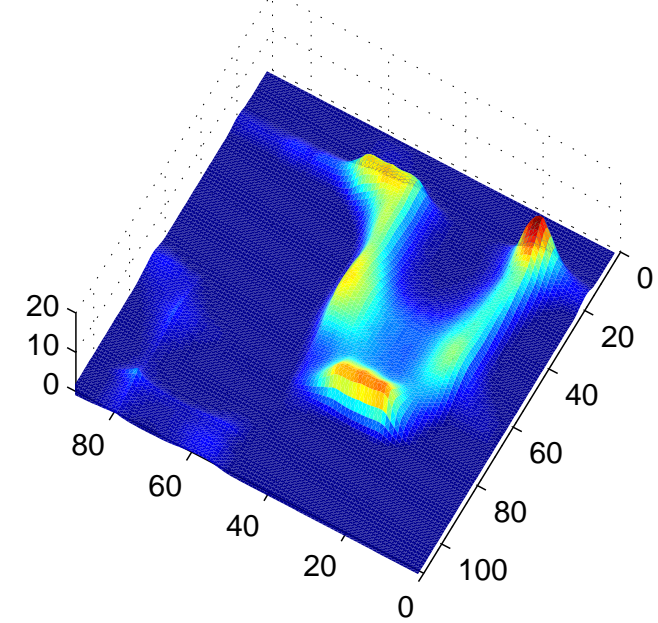

Figure 5.29 Three dimensional images of the letter "V" of a) processed data and b) the deblurred image. 
Figure 5.30, Figure 5.31 and Figure 5.32 show another example of raw data, processed measurements and 3D images of letter "T".
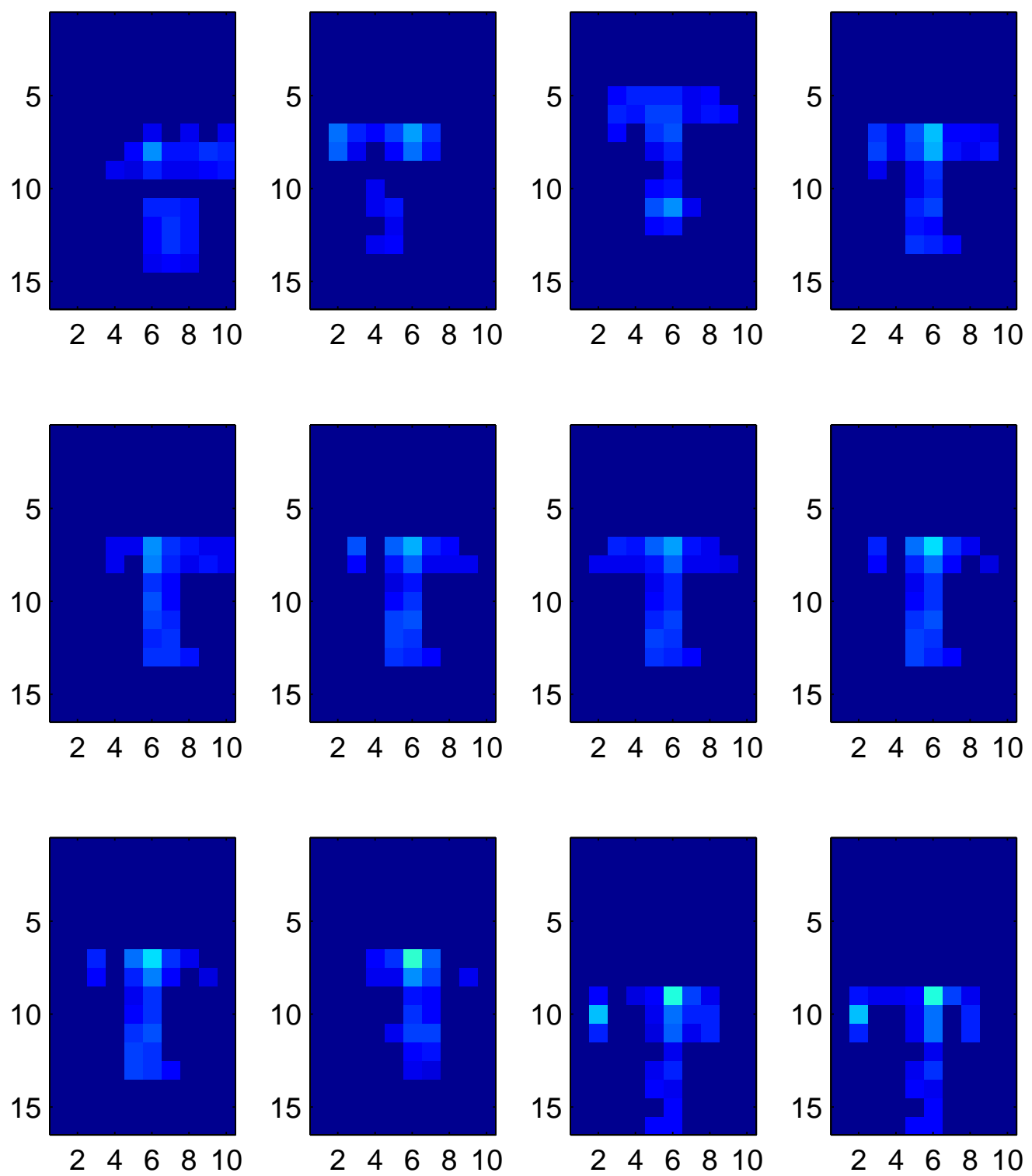

Figure 5.30 Set of 12 measurements of the letter "T" using a Thru mode FSR array. 


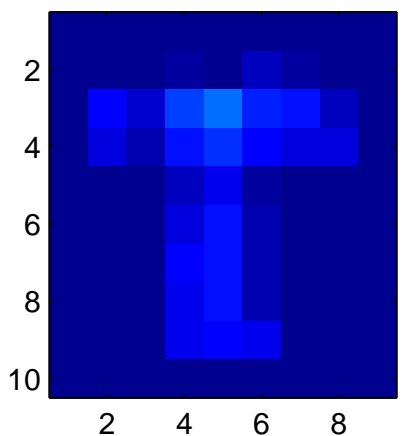

(a)

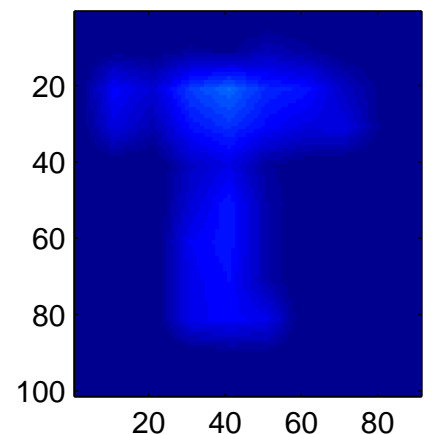

(b)

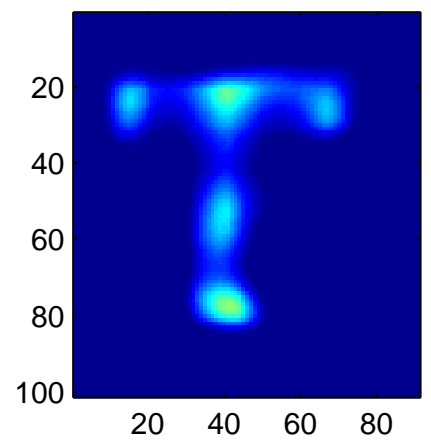

(c)

Figure 5.31 Results produced from multiples images of the letter "T": a) averaged image; b) processed data with PREA where $L=10$; and c) the deblurred image.

(a) Processed Data

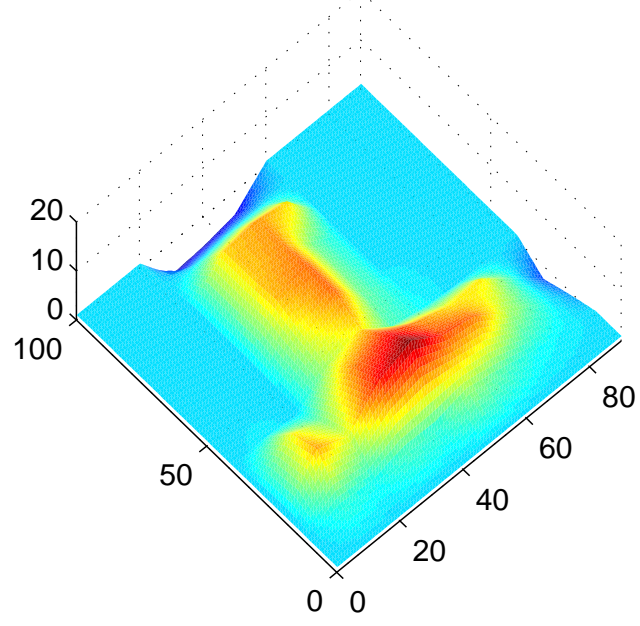

(b) Deblurred Image

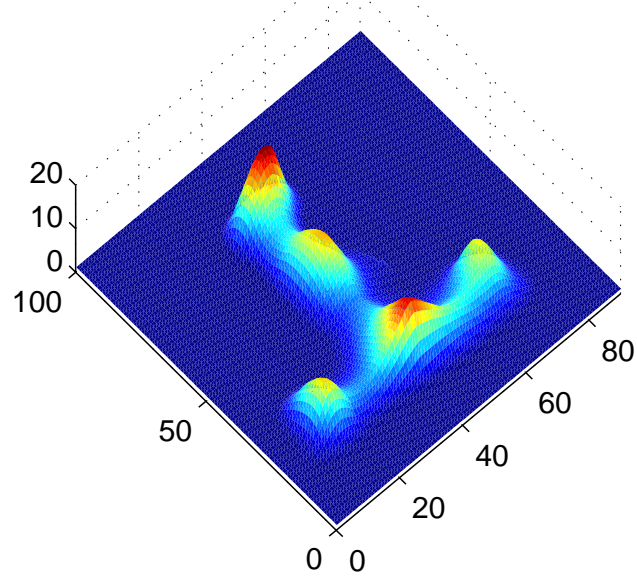

Figure 5.32 Three dimensional images of the letter "T" of a) processed data and b) the deblurred image. 


\subsubsection{Reconstruction from Partial Information of Measurements}

One other application of the proposed algorithm is to combine partial information from multiple measurements to synthesize the complete shape of the object. This case is focused on identifying the geometric shape of the object instead of force distribution. Due to of the size of the object, it was not possible to translate it on the surface of the tactile sensor. Forces with the same magnitude are applied at different locations in order to get all the information to determine the shape of the targeted object. The collected images with forces applied at various locations are illustrated in Figure 5.33.
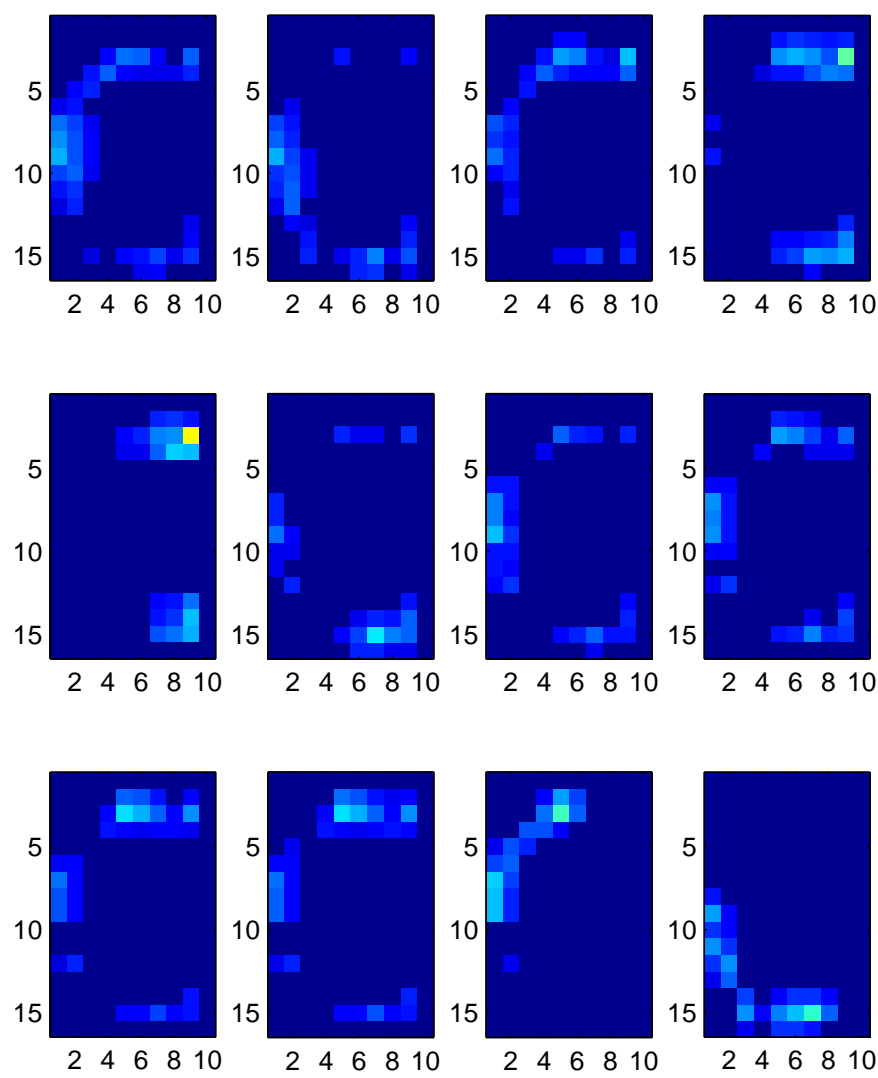

Figure 5.33 Set of 12 measurements of the letter "C" using a Thru mode FSR array. 
To synthesize the complete picture of the object, the total force is applied and assigned in different locations to the corresponding sampled measurements. Thus, sensors with zero readings will not be averaged to emphasize the geometric shape. From Figure 5.34 and Figure 5.35, it is clear that the object of letter C can be reconstructed successfully.
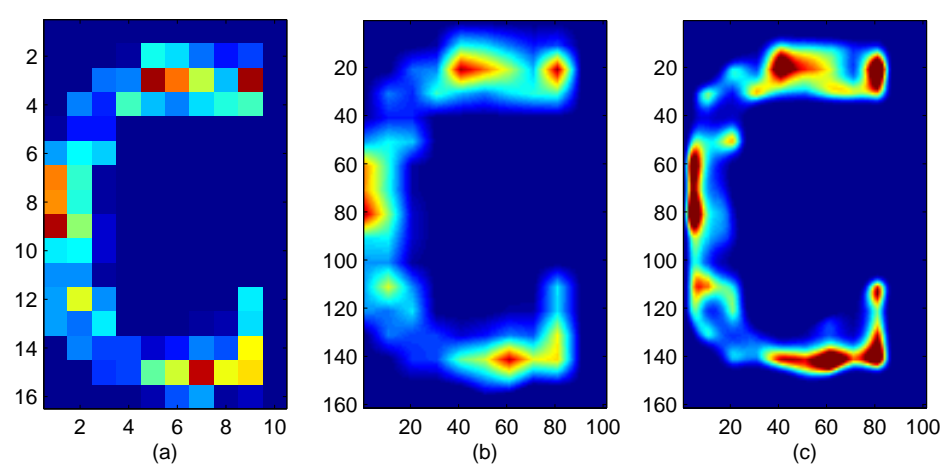

Figure 5.34 Results produced from multiple images of the letter "C": a) averaged image; b) processed data with PREA where $L=10$; and c) the deblurred image

(a) Processed Data

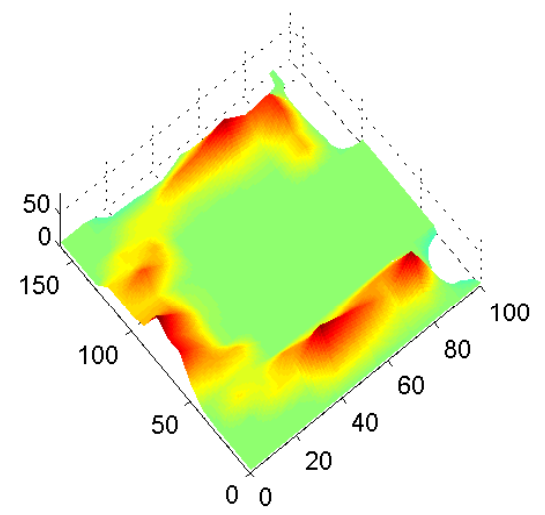

(b) Deblurred Data

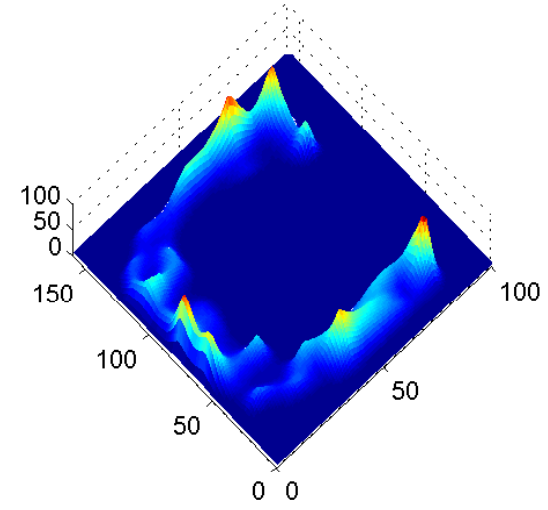

Figure 5.35 Three dimensional images of the letter "C" of a) processed data and b) the deblurred image. 
In short, the proposed method enables the shape of the objects to be identified in great details even when only partial information is available. The deblurred images are a close approximation of the actual shape of the targeted objects. Every edge, angle and length are identified clearly. Therefore, the resolution of a Thru mode FSR array can be enhanced by the implementation of a super resolution algorithm. Such method can be applied to integrate multiple images in nanometer scale to synthesize a much longer image with indentions marked at known locations as well. 


\section{CHAPTER 6}

\section{CONCLUSION}

\subsection{Summary of Research}

The purpose of this research was to propose an integrated tactile system able to work as a synthetic skin, which can provide data of force distribution, weight, location and the geometric shape of an object. The main part of such kind of system is the tactile sensor. In this study, the adopted sensor was a Thru Mode FSR array. This device has the advantages of easy to deploy and its cost is minimum in comparison with other sophisticated sensors. However, due to the limited resolution of the tactile sensor, though the acquisition time is minimum but the acquired information is not good enough to identify the shape of the object placed on the array.

This problem can be easily solved by replacing the sensor with a better one, but the cost of hardware and the corresponding acquisition time need to be compromised as well. In particular, if a sensor with higher number of elements is used, it takes longer time to read all the sensors. Furthermore, the size of the external circuit has to be bigger, and the required input/output ports of analog signals will be increased. As a result, another solution to increase the resolution has to be proposed.

This research proposed to use a super resolution algorithm to enhance the resolution of a tactile sensor without compromising the acquisition time, cost and complexity of the external circuit. In this study, the model of the Thru mode FSR was obtained experimentally and statistically. With the result, a mathematical approximation of the corresponding tactile sensor has also been identified. An external circuit and DAQ station were setup 
accordingly. The designed DAQ system can read all the 160 sensor in within 18 seconds. In the third part a Proposed Resolution Enhancement Algorithm (PREA) was developed, which can used for single images or multiple sets of images. Based on the results, the PREA shows an extraordinary performance when multiple images of the same targeted object are processed. The algorithm integrates five procedures: 1) The collection of all the data; 2) Identification of the contrast map; 3) Translation of the multiple images to a common reference coordinate; 4) Take the average of the images to refine the resolution of processed result and 5) Deblurring of images. Several objects were then tested to validate the effectiveness of the PREA with the Thru mode FSR array. The results of processed images show clearly the shape of targeted objects.

The main contribution of this study was the implementation of a measurement system integrating a tactile sensor array, DAQ station and a PREA. It provides a skin like platform that can acquire, process, and identify measured information. This system can be used as a synthetic skin insomuch as it is capable to accurately read external forces, identify the weigh of a certain object and the geometric shape of the object. All this features are achieved with a extremely low resolution device and without increasing the acquisition time, cost and external circuitry. 


\subsection{Future Directions}

The purpose of this research was achieved satisfactorily. The method PREA can effectively enhance the resolution images acquired by a low resolution tactile sensor. With the current results, further investigations will be carried out. For instance: the multiple images generated by the tactile sensor can be perturbated by some tiny translations and rotations. It is probable that the object can be placed on the surface of the tactile sensor with a slight rotation. Thus, calibration method needs to be proposed to identify the angle of rotation and to align all images to a common coordinate.

It is desirable to increase the covered area with three more sensor arrays so that the initial resolution becomes $32 \times 20$ pixels. This will allow to work with bigger objects. It is expected that better results of object identification can be achieved. The proposed algorithm will also be applied to other platforms for different applications, such as AFM images and scanning of 3D images in the future. 


\section{BIBLIOGRAPHY}

[1] M. Cutkosky, R. Howe, and W. Provancher. Springer handbook of robotics, chapter 19: Force tactile sensors. Springer Berlin Heidelberg, pages 455-476, 2008.

[2] M.H Lee and H.R Nicholls. Review article tactile sensing for mechatronicsa state of the art survey. Mechatronics, pages $1-31,1999$.

[3] The biotac tactile sensor, http://www.syntouchllc.com/products/biotac/, September 2013.

[4] K. Takei, T. Takahashi, J.C. Ho, H. Ko, A.G. Gillies, P.W. Leu, R.S. Fearing, and A. Javey. Nanowire active-matrix circuitry for low-voltage macroscale artificial skin. Nature Materials, pages 821-826, 2010.

[5] S.C. Mannsfeld, B.C-K. Tee, R.M. Stoltenberg, C.V.H-H. Chen, S. Barman, B.V.O. Muir, A.N. Sokolov, C. Reese, and Z. Bao. Highly sensitive flexible pressure sensors with microstructured rubber dielectric layers. Nature Materials, pages 859-864, 2010.

[6] M. Segev-Bar, A. Landman, M. Nir-Shapira, G. Shuster, and H. Haick. Tunable touch sensor and combined sensing platform: Toward nanoparticle-based electronic skin. ACS Applied Materials and Interfaces, pages 5531-5541, 2013.

[7] H. Yousef, M. Boukallel, and K. Althoefer. Tactile sensing for dexterous in-hand manipulation in roboticsa review. Sensors and Actuators A: Physical, pages 171 - 187, 2011.

[8] M.I. Tiwana, S.J. Redmond, and N.H. Lovell. A review of tactile sensing technologies with applications in biomedical engineering. Sensors and Actuators A: Physical, 179(0):17 - 31, 2012. 
[9] D.D. Rossi. Artificial tactile sensing and haptic perception. Measurement Science and Technology, pages 1003-1016, 1991.

[10] S. Najarian, J. Dargahi, and A.A. Mehrizi. Artificial Tactile Sensing in Biomedical Engineering. The McGraw-Hill Companies, Inc., 2009.

[11] R.A. Brookhuis, T.S.J. Lammerink, R.J. Wiegerink, M.J. de Boer, and M.C. Elwenspoek. 3d force sensor for biomechanical applications. Sensors and Actuators A: Physical, pages $28-33,2012$.

[12] A. Petropoulos, G. Kaltsas, D. Goustouridis, and E. Gogolides. A flexible capacitive device for pressure and tactile sensing. Procedia Chemistry, pages 867-870, 2009.

[13] J.A. Dobrzynska and M.A.M. Gijs. Flexible polyimide-based force sensor. Sensors and Actuators A: Physical, pages 127 - 135, 2012.

[14] G. De Maria, C. Natale, and S. Pirozzi. Force/tactile sensor for robotic applications. Sensors and Actuators A: Physical, pages 60 - 72, 2012.

[15] L. Seminara, M. Capurro, P. Cirillo, G. Cannata, and M. Valle. Electromechanical characterization of piezoelectric pvdf polymer films for tactile sensors in robotics applications. Sensors and Actuators A: Physical, pages 49 - 58, 2011.

[16] J. Dargahi. A piezoelectric tactile sensor with three sensing elements for robotic, endoscopic and prosthetic applications. Sensors and Actuators A: Physical, pages 23 - 30, 2000.

[17] .M. Ko and E. Aka. Design of a piezoelectric based tactile sensor with bio-inspired micro/nano-pillars. Tribology International, pages 321 - 331, 2013.

[18] M.-Y. Cheng, C.-M. Tsao, Y.-Z. Lai, and Y.-J. Yang. The development of a highly twistable tactile sensing array with stretchable helical electrodes. Sensors and Actuators A: Physical, pages 226 - 233, 2011. 
[19] Dynamic tire tread measurement system, http://www.pressureprofile.com, September 2013.

[20] How suretouch works, http://www.suretouch.us/consumers/how-it-works, September 2013.

[21] Body mapping applications, http://www.sensorprod.com/bodymapping.php, September 2013 .

[22] R.S. Cok, R.P. Bourdelais, and C.J. Kaminsky. Flexible resistive touch screen, us 7081888 b2. 2016.

[23] P.S. Giro, P.M. Pinto Ramos, O. Postolache, and J.M. Dias Pereira. Tactile sensors for robotic applications. Measurement, pages 1257 - 1271, 2013.

[24] H.-K. Kim, S. Lee, and K.-S. Yun. Capacitive tactile sensor array for touch screen application. Sensors and Actuators A: Physical, pages 2 - 7, 2011.

[25] T.V. Wilson, N. Chandler, W. Fenlon, and B. Johnson. iphone touch screen, http://electronics.howstuffworks.com/iphone1.htm, September 2013.

[26] Shadow dextereous hand, http://www.shadowrobot.com/, September 2013.

[27] H. Iwata and S. Sugano. Design of human symbiotic robot twendy-one. IEEE International Conference on Robotics and Automation, pages 580-586, 2009.

[28] S.E. El-Khamy, M.M. Hadhoud, M.I. Dessouky, B.M. Salam, and F.E.A. El-Samie. New techniques to conquer the image resolution enhancement problem. Progress in electromagnetics Research B, pages 13-51, 2008.

[29] Z. Dengwen. An edge-directed bicubic interpolation algorithm. 3rd International Congress on Image and Signal Processing, pages 1186 - 1189, 2010. 
[30] J. Chu, J. Liu, J. Qiao, X. Wang, and Y. Li. Gradient-based adaptive interpolation in super-resolution image restoration. Signal Processing 9th International Conference on Beijing 2008, pages 1027 - 1030, 2008.

[31] J. Yang, J. Wright, T.S. Huang, and Y. Ma. Image super-resolution via sparse representation. IEEE Transactions, Image Processing, pages 2861 - 2873, 2010.

[32] Fsr 101 - the basics, http://sensitronics.com/fsr101.htm, September 2013.

[33] M.F. Barnsley. Fractals everywhere. San Diego, CA: Academic Press, 1988.

[34] Y. Bresler and A. Macovski. Exact maximum likelihood parameter estimation of superimposed exponential signals in noise. IEEE Acoustics, Speech and Signal Processing, pages $1081-1089,1986$.

[35] Y. Zhang, D. Zhao, J. Zhang, R. Xiong, and W. Gao. Interpolation-dependent image downsampling. IEEE Image Processing, pages 3291 - 3296, 2011.

[36] M.M. Khrushchov and E.S. Berkovich. Methods of determining the hardness of very hard materials: The hardness of diamond. Industrial Diamond Review, pages 42-49, 1951.

[37] M.H. Cheng, T.-C Chiu, and R. Reifenberger. Fractal compression and adaptive sampling: reducing the image acquisition time in scanning probe microscopy. Scanning, pages 463-473, 2008.

[38] M.H. Cheng and G. T.-C Chiu. Fractal compression and adaptive sampling for atomic force microscopy. International Conference on Advanced Intelligent Mechatronics 2005, pages 99-104, 2005. 